INTERNATIONAL MONETARY FUND

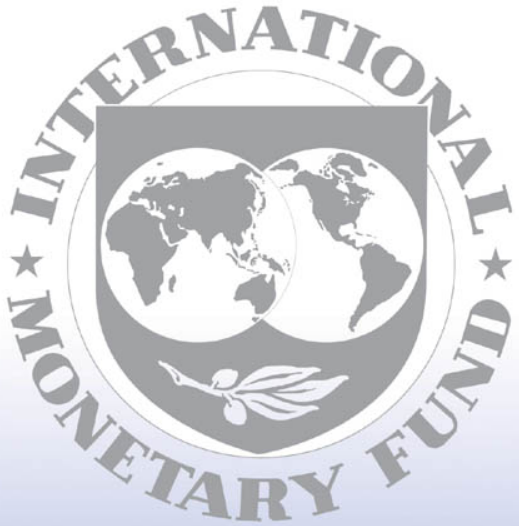

Staff

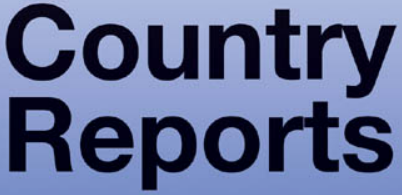




\title{
Ghana: 2009 Article IV Consultation and Request for a Three-Year Arrangement Under the Poverty Reduction and Growth Facility_Staff Report; Staff Supplement; Staff Statement; Public Information Notice and Press Release on the Executive Board Discussion; and Statement by the Executive Director for Ghana
}

\begin{abstract}
Under Article IV of the IMF's Articles of Agreement, the IMF holds bilateral discussions with members, usually every year. In the context of a combined discussion of the 2009 Article IV consultation with Ghana and request for a three-year arrangement under the Poverty Reduction and Growth Facility, the following documents have been released and are included in this package:
\end{abstract}

- $\quad$ The staff report for the combined 2009 Article IV Consultation and Request for a Three-Year Arrangement Under the Poverty Reduction and Growth Facility, prepared by a staff team of the IMF, following discussions that ended on May 22, 2009, with the officials of Ghana on economic developments and policies. Based on information available at the time of these discussions, the staff report was completed on June 30, 2009. The views expressed in the staff report are those of the staff team and do not necessarily reflect the views of the Executive Board of the IMF.

- A staff supplement on the joint IMF/World Bank debt sustainability analysis.

- $\quad$ A staff statement of July 15, 2009 updating information on recent economic developments.

- $\quad$ A Public Information Notice (PIN) and a Press Release, summarizing the views of the Executive Board as expressed during its July 15, 2009, discussion of the staff report that concluded the Article IV consultation and the request, respectively.

- $\quad$ A statement by the Executive Director for Ghana.

The documents listed below have been separately released.

Letter of Intent sent to the IMF by the authorities of Ghana*

Memorandum of Economic and Financial Policies by the authorities of Ghana*

Technical Memorandum of Understanding*

*Also included in Staff Report

The policy of publication of staff reports and other documents allows for the deletion of marketsensitive information.

\footnotetext{
Copies of this report are available to the public from

International Monetary Fund • Publication Services

$70019^{\text {th }}$ Street, N.W. • Washington, D.C. 20431

Telephone: (202) 623-7430 • Telefax: (202) 623-7201

E-mail: publications@imf.org • Internet: http://www.imf.org
}

\section{International Monetary Fund \\ Washington, D.C.}


This page intentionally left blank 


\title{
INTERNATIONAL MONETARY FUND
}

\author{
GHANA \\ Staff Report for the 2009 Article IV Consultation \\ and Request for a Three-Year Arrangement Under the \\ Poverty Reduction and Growth Facility
}

Prepared by the African Department

(in consultation with other departments)

Approved by Michael Atingi-Ego and Dominique Desruelle

June 30, 2009

Discussions. Discussions for the 2009 Article IV consultation and on the authorities' request for a PRGF arrangement were held in Accra during May 11-22, 2009. The staff team comprised Messrs. Allum (head), Erasmus, Kovanen (all AFR), Jarmuzek (SPR) and Ms. Lukonga (MCM). The mission was supported by Mr. McIntyre (resident representative); Mr. Kwakye, Advisor to the Executive Director for Ghana, joined the discussions. The staff met with Minister of Finance Duffuor, Bank of Ghana Governor Acquah, other senior officials, and representatives of parliament, labor unions, the private sector, NGOs, and the donor community. The mission liaised closely with the World Bank. A press release was issued at the end of the mission.

PRGF arrangement. The Ghanaian authorities request a three-year PRGF arrangement with access of 105 percent of quota (SDR 387.45 million). This supports a program of fiscal consolidation to tackle macroeconomic instability, which is currently the main obstacle to sustained strong growth and poverty reduction. A broad agenda of structural reforms will strengthen fiscal institutions ahead of the start of oil production in 2011.

Exchange regime. Ghana maintains a floating exchange rate regime and an exchange system free of restrictions on payments and transfers for current international transactions. 


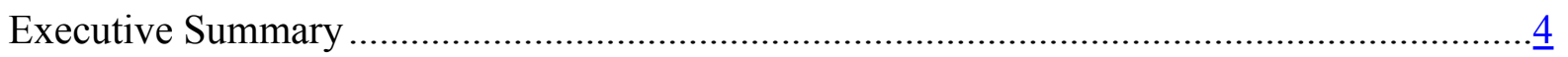

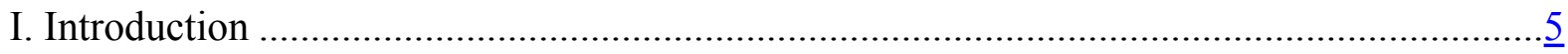

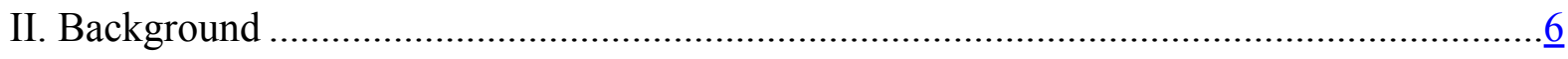

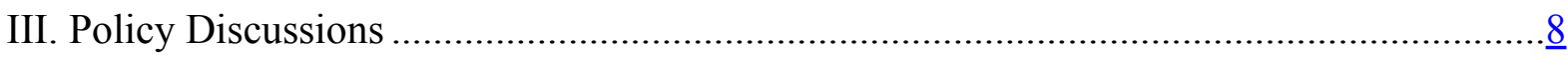

A. Growth Prospects and Risks …………..........................................................

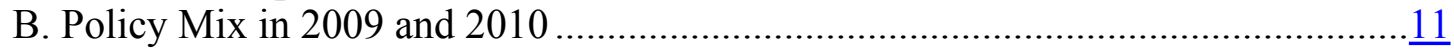

C. Strategy for Fiscal Consolidation....................................................................

D. Monetary, Exchange Rate, and Financial Sector Policies ……..............................14

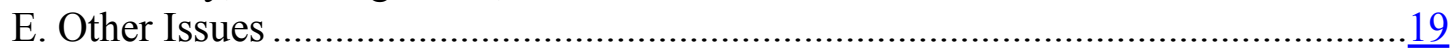

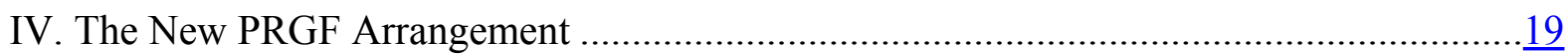

A. Program Objectives and Design......................................................................19

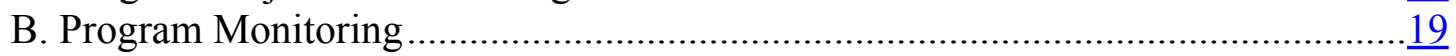

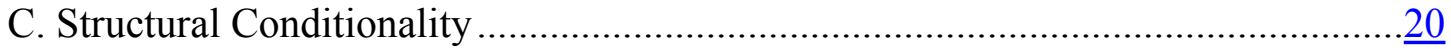

D. Program Financing ........................................................................................

E. Program Risks and Mitigation....................................................................

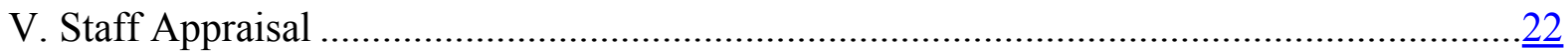

Boxes

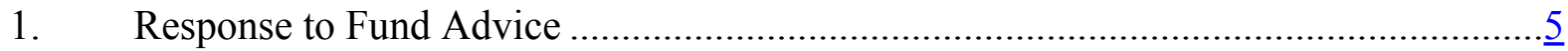

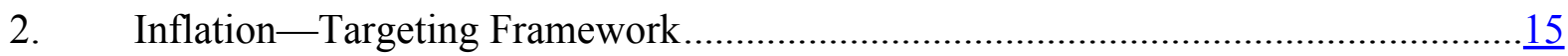

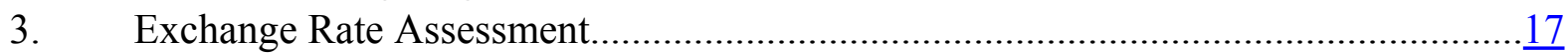

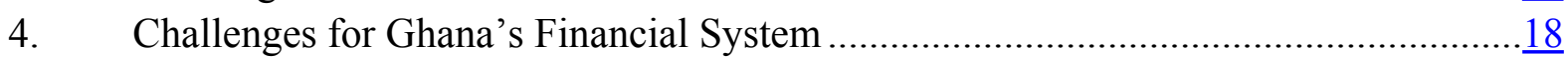

Figures

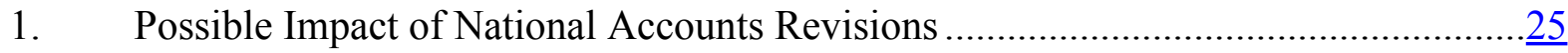

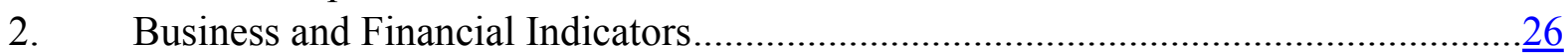

Tables

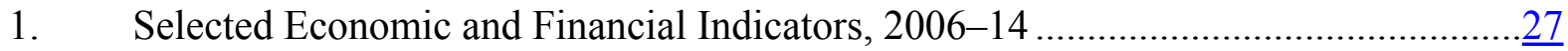

2a. Summary of Central Government Budgetary Operations, 2006-14 ...........................

2b. Summary of Central Government Budgetary Operations, 2006-14............................29

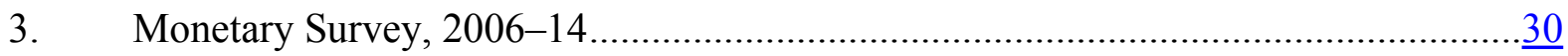




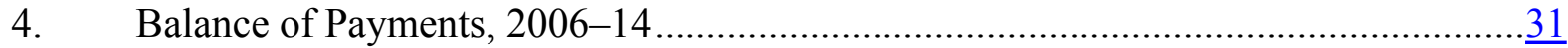

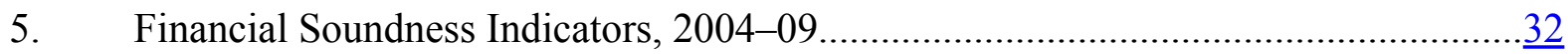

6. External Financing Requirements and Sources, 2006-14 ….................................33

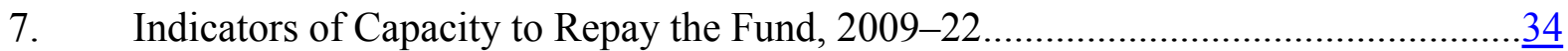

8. Proposed Schedule of Disbursements Under the PRGF Arrangement, 2009-12 …..... 35

9. Selected Indicators on the Millennium Development Goals, 1990-2007 ....................36

Appendix

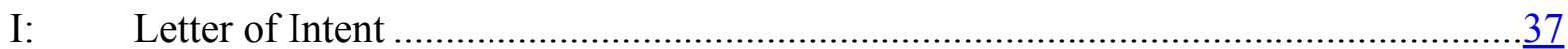

Attachment I. Memorandum of Economic and Financial Policies, 2009-12 …......39

Attachment II. Technical Memorandum of Understanding................................... 


\section{EXECUTIVE SUMMARY}

- Highly expansionary fiscal policy destabilized the economy in 2008 . With the fiscal deficit rising to 14.5 percent of GDP, inflation rose to 20 percent, the currency depreciated by 50 percent against the dollar, and official reserves fell to 2 months' import cover.

- The 2009 budget seeks fiscal consolidation. With expenditure restraint and higher grants, the budget targets a 9.4 percent of GDP deficit. Cash management and expenditure monitoring have been strengthened, and priorities for tax policy and revenue management have been identified.

- The mission reviewed the economic outlook and the new government's program. Ghana appears likely to fare better than many countries during the 2009 global recession, reflecting an improved terms of trade and limited banking, manufacturing, or other exposures. The staff were more pessimistic about growth than the authorities, forecasting an expansion of $4 \frac{1}{2}$ percent in 2009 , rising to 5 percent in 2010 .

- Review of the 2009 budget outlook showed a projected deficit over-run. Based on the staff's growth forecast and other updates, the fiscal deficit was projected at 10.4 percent of GDP, on unchanged policies. The government have cut tax exemptions, identified wage savings, and are adopting a temporary profit tax surcharge to meet the original deficit target. They also stand ready to tighten spending further in the event of unexpected shortfalls in growth and revenues.

- Further fiscal measures of about $3 \frac{1}{2}$ percent of GDP will be needed in the 2010 budget. Utility prices will likely need to rise to offset rising power generation costs, and revenue mobilization options should be explored.

- The inflation targeting framework will need to be robust in 2009-10. Despite a tighter fiscal stance, inflation expectations are high, reinforced by continuing currency depreciation. With real interest rates only moderately positive and resumed strength in global oil prices, there is a strong case for further monetary policy tightening.

- Exchange rate flexibility should be maintained. A large depreciation against the dollar has translated into only modest correction in the real exchange rate. The authorities see the currency as appropriately valued, while staff see risks of continuing modest overvaluation. Given possible needs for further correction, tight monetary policy would help limit the inflation pass-through.

- The financial sector has been resilient to the initial impact of the global financial crisis. But risks have accumulated in an environment of rapid banking expansion, strong competition, and increased risk taking. A recent deterioration in loan books could continue, based on rising funding costs and currency depreciation. This is mitigated by steps to increase minimum capital.

- The authorities have requested a PRGF with access of 105 percent of quota. It would support a program focused on multi-year fiscal consolidation and public debt stabilization. Key fiscal institutions (both revenue and expenditure management) would be strengthened ahead of the start of oil production in 2011. Staff supports this request. 


\section{INTRODUCTION}

\section{Ghana completed a Poverty Reduction and Growth Facility (PRGF)} arrangement in October 2006. Under the PRGF, it achieved strong growth, single-digit inflation, major debt reduction, ${ }^{1}$ and a decline in the poverty rate from 39 percent (1998-99) to 28 percent (2005-06); other social welfare indicators also improved. Fiscal stability was elusive, however, with the deficit rising sharply by end-2006.

\section{Discussions in 2007-08 on a program to be supported by the Policy Support} Instrument (PSI) did not advance. Responsiveness to the Fund's 2008 surveillance advice on fiscal management was also limited (Box 1). The most fruitful areas of collaboration in 2007-08 centered on Fund staff advice on building access to global financial markets and developing the framework for an inflation targeting regime.

\section{Box 1. Ghana: Response to Fund Advice}

Fiscal policy: A central message of the 2008 Article IV consultation concluded by the Board on June 30, 2008 was the urgency of fiscal tightening to avoid deteriorating debt dynamics and risks to external sustainability. In practice, a highly expansionary fiscal stance was maintained through the end-2008 elections, with large over-runs in public sector wages.

Monetary and exchange rate policy: In line with Fund advice, the Bank of Ghana continued to tighten monetary policy through the second half of 2008 and into early-2009. However, given the expansionary fiscal stance, this was not sufficiently aggressive to stem inflation. On exchange rate policy, the greater flexibility in the exchange rate since mid-2008 was in line with Fund advice.

Structural reforms: Little progress was made in 2008 on macro-critical reforms to public financial management (PFM) and the civil service.

3. The macroeconomic dialogue has strengthened under the new government. At the authorities' request, a staff team visited Accra in February 2009 to support preparatory work for the 2009 budget (adopted in March), and technical assistance was requested on tax policy, revenue administration, and natural resource taxation (with missions completed in April-June).

4. The authorities have requested a new PRGF arrangement. This will underpin fiscal consolidation and a program of structural fiscal reforms ahead of Ghana's move to oil producer status starting in 2011 (Section IV).

\footnotetext{
${ }^{1}$ Under the Heavily Indebted Poor Countries Initiative (HIPC) and Multilateral Debt Relief Initiative (MDRI).
} 


\section{BACKGROUND}

5. Economic growth rose to a two-decade high of 7.3 percent in 2008. This reflected expansionary fiscal policies combined with an upswing in private sector activity based on strong credit expansion, buoyant remittances, and strong agricultural yields. For 2009, growth is projected to slow, based on policy tightening and spillovers from the global recession.

6. Inflation has risen on account of external shocks and strong domestic demand. After falling briefly to single-digits in 2006, inflation rose through 2007-08, reflecting global food and fuel price shocks, strong domestic demand, and the pass through from currency depreciation. In early-2009, inflation stabilized in the 20 percent range. With easing demand pressures, inflation is projected to decline to $14 \frac{1}{2}$ percent by end- 2009 .

\section{Presidential and parliamentary elections were a factor behind fiscal slippages in} 2008. The fiscal deficit surged to 14.5 percent of GDP, reflecting rapid public spending growth, with capital spending, energy subsidies, and wage and salaries each rising by more than 1 percentage point of GDP. Following earlier power shortages, new investments were made in thermal power capacity; large subsidies were incurred when electricity tariffs were not adjusted to reflect the rising cost of power generation; and public sector salaries were increased, on average, by more than 30 percent (15 percent in real terms). The 2008 fiscal deficit drew on exceptional foreign financing amounting to 7.3 percent of GDP, comprising resources from the late-2007 Eurobond issue and proceeds from the sale of Ghana Telecom. ${ }^{2}$ In addition, the central bank provided financing equivalent to more than 3 percent of GDP.

\section{The 2009 budget targets a deficit of 9.4 percent of GDP, largely based on} spending cuts and higher grant receipts. Following the completion of power sector and other investments, capital spending has been cut by 3 percent of GDP (Text Table 1). Energy sector subsidies are also projected to decline by more than 1 percent of GDP, in line with global oil prices. These savings are partly offset, however, by higher interest costs and a larger provision for clearing domestic arrears. Updated fiscal projections developed during the May mission suggested a potential deficit overrun of 1 percent of GDP. To maintain the 2009 deficit target the authorities have cut tax exemptions, identified wage bill and investment savings, and are adopting a temporary increase in the profit tax for selected industries (MEFP, $\Upsilon 24)$.

\footnotetext{
${ }^{2}$ The US\$750 million yield from issuing a debut Eurobond in late-2007 was largely spent in 2008.
} 
Text Table 1. Fiscal Adjustment Under 2009 Program

\begin{tabular}{|lrrrr|}
\hline (Percent of GDP) & 2008 & $\underline{2009}$ & $\underline{2009}$ & $\begin{array}{c}\text { Adjust- } \\
\text { ment } 1 /\end{array}$ \\
\hline Revenues & 22.8 & 23.7 & 23.9 & 1.1 \\
Grogram & 4.7 & 6.1 & 6.0 & 1.3 \\
Total expenditure, of which: & 42.0 & 39.3 & 39.4 & -2.6 \\
$\quad$ Wages and salaries 2/ & 11.3 & 10.9 & 10.9 & -0.4 \\
Subsidies & 1.5 & 0.3 & 0.3 & -1.2 \\
Interest costs & 3.9 & 3.9 & 4.3 & 0.4 \\
Capital spending & 15.7 & 12.9 & 12.6 & -3.1 \\
Arrears clearance & 1.0 & 2.7 & 2.7 & 1.7 \\
Fiscal deficit & 14.5 & 9.4 & 9.4 & -5.1 \\
\hline
\end{tabular}

Source: Official data; and Fund staff projections.

1/ Fiscal adjustment in program, relative to GDP, including June 2009 measures.

2/ The 2009 data have been adjusted to ensure comparable coverage to 2008 .

9. Monetary policy was tightened in response to rising inflation. The Bank of Ghana increased its benchmark lending rate by 6 percentage points between October 2007 and February 2009, and market conditions tightened by more, as reflected in interbank and treasury bill rates. The policy rate was left unchanged in May 2009 on an assessment that inflationary pressures were easing. Despite rate increases, the real prime rate has been negative since early-2008 (based on backward-looking inflation), while the real interbank rate remains in the 0-2 percent range. The banking sector has continued to register strong asset growth, with private sector credit up 56 percent in the year to April 2009, only partly due to valuation changes on foreign currency-denominated loans. This expansion continues to reflect local deposit mobilization, with broad money up 28 percent on the same basis.

Ghana: Real Interest Rates, 2005-2009 1/

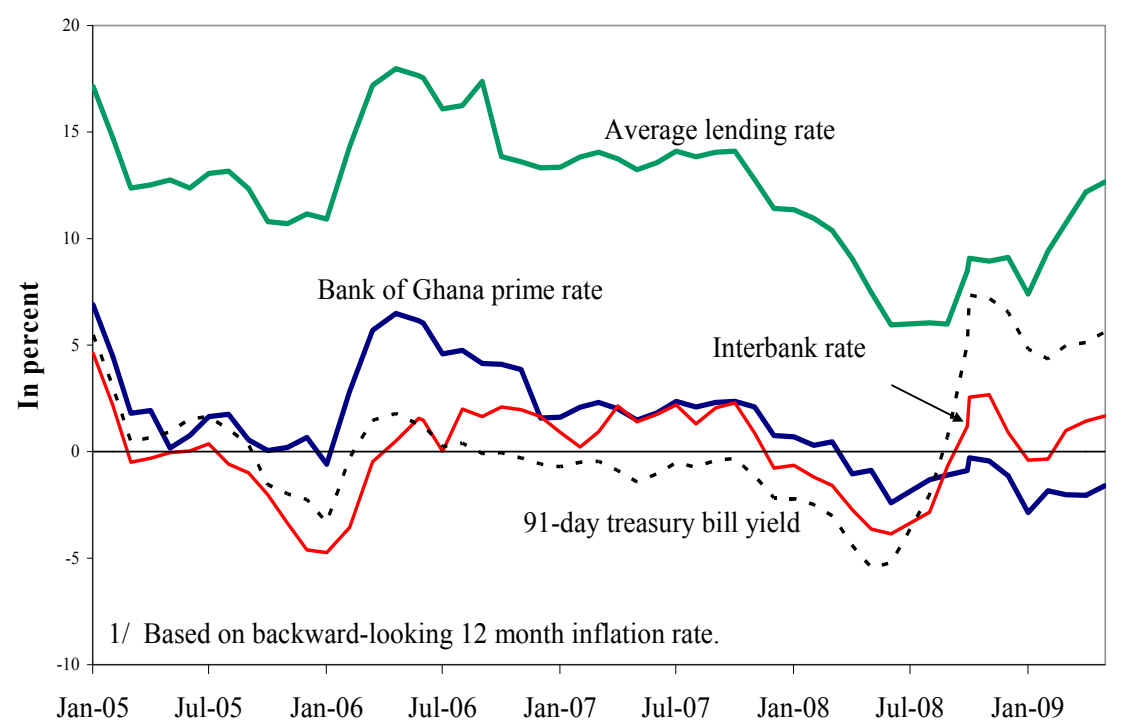


10. Ghana's overall financial system remains stable. The regulatory and supervisory framework is strong, backed by a modern payment and settlement infrastructure. Financial Soundness Indicators (FSIs) point to a banking system that is liquid and with capital above statutory levels. However, some recent deterioration is evident: non-performing assets are high and rising (9.6 percent in March 2009); profitability is declining; and a few banks are financially strained. The Bank of Ghana has required banks to raise their minimum capital to GH $₫ 60$ million (about $\$ 40$ million) to allow them to play a larger role in the rapidly growing economy. ${ }^{3}$ The non-banking sector (insurance, pensions and capital markets) has continued to perform well.

\section{Ghana's external current account deficit widened to 19 percent of GDP in 2008,} up from 12 percent of GDP a year earlier. This largely reflected a 33 percent increase in non-oil imports values, driven by strong domestic demand. Financing was provided through an increased external capital account surplus, buoyed by Ghana Telecom privatization proceeds ( $5 \frac{1}{2}$ percent of GDP), and a draw-down of gross international reserves from US $\$ 2.8$ billion to US $\$ 2.0$ billion. Reflecting the latter, gross reserve cover declined from 2.7 to 2.2 months of projected import cover.

\section{Exchange rate flexibility has increased since mid-2008. Reflecting Ghana's} macroeconomic imbalances and declining foreign exchange inflows, the exchange rate depreciated about 50 percent against the dollar during 2008 and the first half of 2009. Much of this adjustment was offset, however, by Ghana's high inflation rate and the appreciation of the dollar in the context of the global financial crisis. Accordingly, the real effective exchange rate in April 2009 was just 8 percent more depreciated than in 2007. Over the recent period, illiquidity in the foreign currency market has been associated with a decline in interbank trading and a widening of spreads.

\section{Policy Discussions}

\section{A. Growth Prospects and Risks}

Discussions focused on the uncertain growth outlook, where staff are less optimistic than the authorities, and see downside risks. In 2011, the start of oil production will boost growth and government revenues, but create new challenges for macroeconomic management.

\section{Growth prospects for 2009-10 are particularly uncertain, reflecting divergent} influences. On the positive side, Ghana is benefiting from a terms of trade improvement projected at 20 percent in 2009, as cocoa and gold prices remain buoyant while oil import costs have declined. In addition, credit growth remains strong and favorable rainfall trends

\footnotetext{
${ }^{3}$ Majority foreign-owned banks are required to meet the target by end-2009, while domestic banks have a deadline of end-2012.
} 
are expected to continue to benefit hydroelectricity and agricultural production. Given these factors, the authorities envisage only a modest slowdown, to a little under 6 percent in 2009. By contrast, the mission expects fiscal tightening to be a significant drag on growth in 2009 . In addition, credit growth is projected to slow, and the global recession is having an impact through the remittances channel, with first quarter inflows down 18 percent on a year earlier. On this basis, the mission projected growth of $4 \frac{1}{2}$ percent in 2009 , consistent with a composite indicator series compiled by the Bank of Ghana (Figure below). For 2010, the mission projected growth of 5 percent, based on an improving external environment. While viewing the staff's forecast as overly pessimistic, the authorities agreed to use it for fiscal planning (Section III.B).

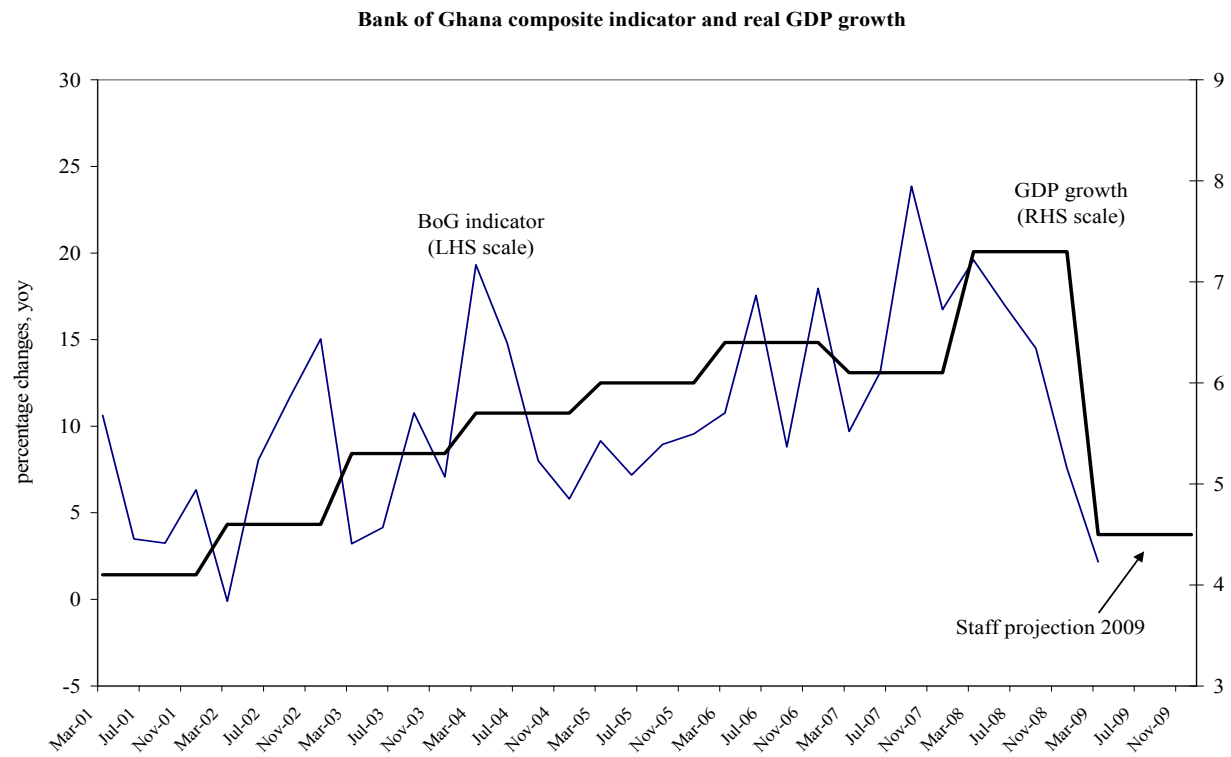

14. From 2011, Ghana will benefit from the start of oil production. The proven reserves are relatively modest at present, with the offshore Jubilee field estimated at 490 million barrels. ${ }^{4}$ However, test results from other fields are favorable, and reserves could rise to 1.5 billion barrels or more (matching Chad and the Republic of Congo). Production from the Jubilee field is planned to start in 2011, with peak production over an initial 5-6 years, followed by a progressive decline. Initial production is projected at about 17 percent of non-oil GDP, with more than one third accruing to government revenues (6-7 percent of GDP in 2011-12). Oil production would largely substitute for oil imports, except in 2011-15, when an exportable surplus is projected (figure overleaf).

\footnotetext{
${ }^{4}$ Pending more definitive information, no allowance has been made for potential gas exports from the Jubilee field.
} 


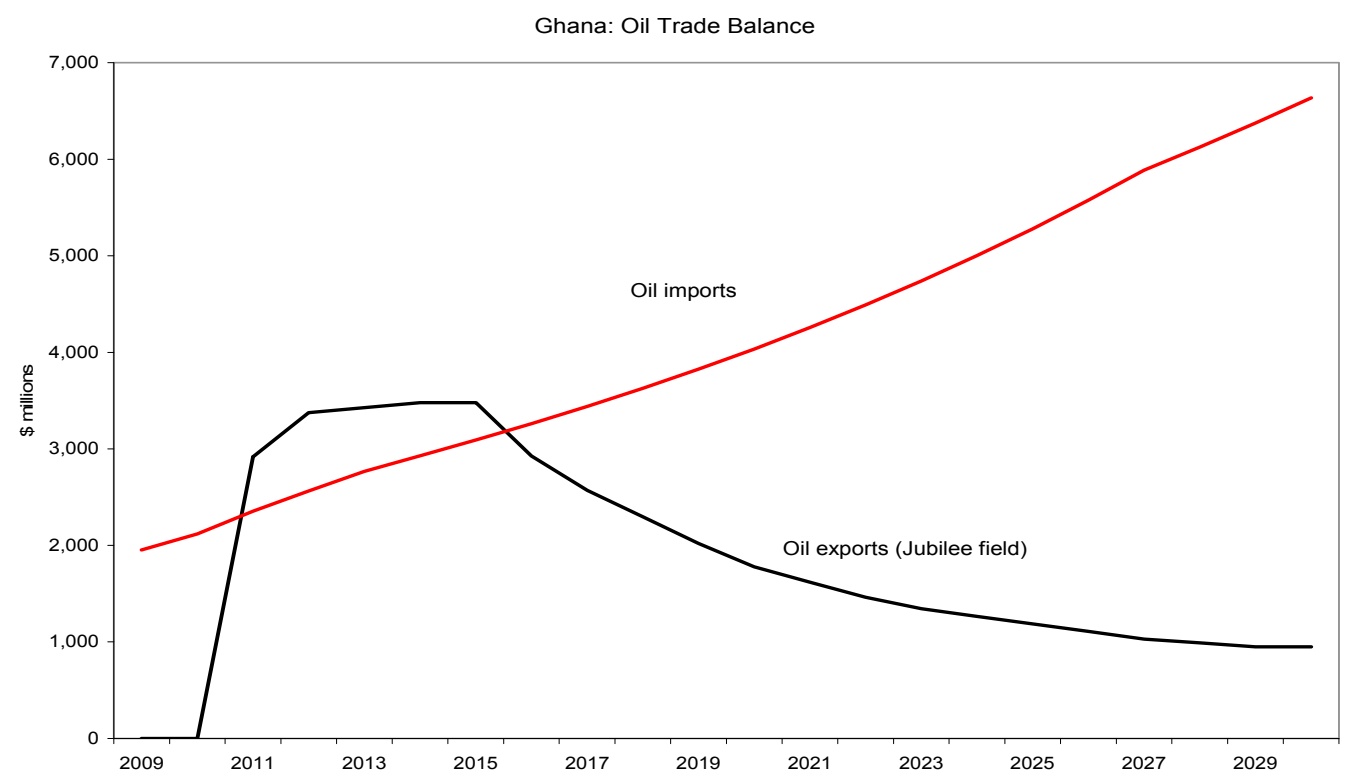

15. Macroeconomic management will need to be alert to the consequences of oil production. The oil sector and related spending of oil revenues will boost demand pressures starting in 2011. On the assumption that demand pressures are prudently managed, the medium-term framework projects a return to robust non-oil growth in the 5-6 percent range, and a decline in inflation to about 5 percent. A projected fiscal deficit of 4 percent on average over the medium-term is projected to result in a small decline in the net debt-GDP ratio. The external current account deficit would increase in 2010 due to oil-related infrastructure imports, before declining over the medium term as oil exports come on stream (Text Table 2).

Text Table 2. Macroeconomic Outlook

\begin{tabular}{|c|c|c|c|c|c|c|c|c|}
\hline & 2007 & 2008 & 2009 & 2010 & 2011 & 2012 & 2013 & 2014 \\
\hline & \multicolumn{8}{|c|}{ (Annual percentage change) } \\
\hline Real GDP & 5.7 & 7.3 & 4.5 & 5.0 & 24.2 & 7.1 & 5.3 & 5.0 \\
\hline Nonoil real GDP & 5.7 & 7.3 & 4.5 & 5.0 & 6.2 & 6.2 & 6.2 & 5.9 \\
\hline \multirow[t]{2}{*}{ Inflation, end-of-period } & 12.7 & 18.1 & 14.6 & 9.2 & 8.0 & 5.0 & 5.0 & 5.0 \\
\hline & \multicolumn{8}{|c|}{ (Percent of GDP) } \\
\hline Overall fiscal balance & -9.2 & -14.5 & -9.4 & -6.0 & -4.5 & -2.8 & -2.1 & -2.1 \\
\hline Net public debt & 51.2 & 58.2 & 66.4 & 66.6 & 66.0 & 63.7 & 60.8 & 58.1 \\
\hline External current account balance & -12.0 & -19.3 & -13.0 & -15.9 & -9.5 & -8.3 & -9.5 & -6.0 \\
\hline Foreign direct investment & 7.1 & 14.3 & 7.7 & 13.1 & 11.3 & 9.9 & 10.8 & 7.5 \\
\hline \multicolumn{9}{|l|}{ Of which: } \\
\hline Divesture-related FDI & 0.8 & 5.6 & 0.0 & 0.0 & 0.0 & 0.0 & 0.0 & 0.0 \\
\hline FDI in offshore oil fields & 0.0 & 0.0 & 2.8 & 7.4 & 6.0 & 3.4 & 3.9 & 0.0 \\
\hline
\end{tabular}

Sources: Official data and Fund staff projections. 
16. Risks to growth are on the downside. Although Ghana is projected to achieve a "soft landing", spillovers from the global recession may prove larger than currently projected, possibly through an intensified decline in remittances or a deterioration in gold or cocoa prices. In addition, a shift by domestic banks to more cautious lending standards in the context of rising non-performing loans could trigger a larger than expected slowdown in private sector investment.

\section{B. Policy Mix in 2009 and 2010}

\section{The authorities' policy framework seeks to reduce inflation while rebuilding the} external reserve position. Reserve and broad money growth are projected to slow to reduce inflation to single-digit rates by end-2010 (Text Table 3). The decline in domestic credit growth is more marked than for overall liquidity, releasing resources to rebuild Ghana's foreign currency reserve position. For 2009, the financial program aims to avoid further losses in net international reserves from end-March levels, while the program for 2010 targets a modest reserve accumulation. The authorities and staff would have normally have preferred a larger near-term reserve build-up, but the additional fiscal adjustment and reduction in net domestic financing needed to accomplish this was not seen as realistic.

Text Table 3. Monetary Framework

\begin{tabular}{|c|c|c|c|c|c|c|c|c|}
\hline & 2007 & 2008 & 2009 & 2010 & 2011 & 2012 & 2013 & 2014 \\
\hline & \multicolumn{8}{|c|}{ (In percentage changes) } \\
\hline Reserve money & 30.5 & 27.1 & 16.5 & 14.9 & 24.3 & 17.0 & 13.2 & 13.8 \\
\hline Of which: Net foreign assets ${ }^{1}$ & 42.9 & -30.9 & 10.0 & 20.9 & 60.0 & 54.8 & 45.2 & 39.9 \\
\hline Broad money & 35.9 & 40.2 & 21.7 & 14.5 & 21.8 & 18.7 & 15.9 & 15.1 \\
\hline Of which: Claims on government (net) ${ }^{2}$ & -9.1 & 22.5 & 5.6 & -3.8 & 0.2 & -0.7 & -0.7 & 0.3 \\
\hline \multicolumn{9}{|l|}{ Memorandum items } \\
\hline Credit to government (percent of GDP) & 7.9 & 13.6 & 13.7 & 10.0 & 7.6 & 6.6 & 5.6 & 5.2 \\
\hline Real credit to private sector & 41.6 & 25.5 & 9.0 & 15.0 & 10.0 & 10.0 & 10.0 & 10.0 \\
\hline Increase in Bank of Ghana NFA (US\$ million) & 566.9 & -800.4 & -404.0 & 200.0 & 862.0 & 1007.0 & 903.7 & 851.8 \\
\hline
\end{tabular}

\section{Strategy for Fiscal Consolidation}

A highly expansionary fiscal stance has contributed to macroeconomic instability. Plans for near-term fiscal consolidation need to be backed by a strategy for using oil wealth.

18. Deficit reduction is the immediate macroeconomic priority. The authorities recognize the destabilizing impact of the fiscal deficit: the 2008 outturn is comparable to levels last seen in 1992-99, when inflation peaked at over 70 percent, and public debt rose from under 50 percent to more than 110 percent of GDP. The planned deficit reduction to $4 \frac{1}{2}$ percent of GDP in 2011 , and subsequently lower, will address inflationary pressures and stem a renewed upturn in public debt. 
19. Fiscal measures in $\mathbf{2 0 0 9}$ are being augmented to preserve the deficit target. The mission welcomed the emphasis on spending restraint in 2009, which will partially reverse the upward trend that contributed to the 2008 deficit. The prompt adoption of measures in June to avoid over-runs in the fiscal deficit are also welcome (MEFP, q24). The authorities have intensified cash management, and in the event of an even larger slowdown in economic growth and revenue collections, are ready to maintain the deficit target through cuts in discretionary spending.

20. The 2010 budget will be challenging. The mission noted that fiscal measures of about $3 \frac{1}{2}$ percent of GDP will be needed to achieve the 6 percent deficit target for 2010 (see Table 2A). On current estimates, this target could largely be achieved by expenditure restraint, including in the public wage bill, combined with higher utility prices to address a projected power sector shortfall. ${ }^{5}$ In practice, however, the authorities will also need to explore options to strengthen revenue mobilization. The fiscal gap is likely to be larger than currently estimated, reflecting the potential need to clear remaining arrears from 2008 (MEFP, ๆ53). Equally, the projected compression of the wage bill in 2010 may not be feasible, given the planned salary structure reform, and the squeeze on capital spending may not be sustainable, given emerging infrastructure needs.

21. Ghana maintains a broad social safety net program. Current protection for the most vulnerable include subsidized electricity tariffs (up to a maximum monthly usage), a cash transfers to qualifying households, school feeding, free maternal health care, grants and subsidies for basic education, and a national youth employment program. The authorities plan to expand cash transfers for qualifying households, provide free school uniforms to children in deprived communities, and free books for pupils in basic public schools. Staff welcomed these measures, which should safeguard low-income groups from fiscal tightening.

22. At the same time, the authorities intend to re-examine budget flexibility. The growth of mandatory and similarly-protected spending commitments limits the space for fiscal maneuver. With a rising wage bill, an expansion of health care transfers in 2007, and the majority of capital spending protected from annual budget review (in the form of trust funds with earmarked financing), the inflexible element of the annual budget has risen from 92 percent of revenues and grants in 2006 to 107 percent in 2009 (Text Table 4). Staff recognized the case for reviewing funding arrangements for capital spending, providing that poverty-reducing programs retain appropriate protection.

\footnotetext{
${ }^{5}$ The fiscal projections reflect unchanged utility tariffs. Based on the global oil price outlook, a depreciating currency, and a projected shift from hydro to more costly thermal power generation, operating shortfalls of the power authority are projected at nearly 3 percent of GDP in 2010. The government's commitment to adopting cost-recovery utility tariffs by end-2009 will therefore be critical for supporting the 2010 budget (MEFP, $\mid 52$ ).
} 
Text Table 4. Mandatory and Inflexible Public Expenditures

\begin{tabular}{lrrrr}
\hline (Percent of GDP, unless otherwise indicated) & 2006 & 2007 & 2008 & 2009 \\
\hline Wages and salaries 1/ & 9.7 & 10.1 & 11.3 & 11.8 \\
Pension, social security, health transfers & 2.2 & 3.8 & 3.2 & 3.8 \\
Interest payments & 3.4 & 3.1 & 3.9 & 4.3 \\
Capital expenditures & 12.4 & 14.4 & 15.7 & 12.6 \\
$\quad$ Foreign-financed and trust funds 2/ & 9.9 & 10.7 & 10.0 & 11.3 \\
$\quad$ Discretionary & 2.5 & 3.6 & 5.7 & 1.3 \\
Memorandum items (\% of revenue and grants) & 92.0 & 96.2 & 103.1 & 107.0 \\
\hline Mandatory and inflexible spending 3/ & & & \\
1/ 2009 wage figure includes "Category I" employment allowances. & \\
2/ Education Fund, Road Fund, District Assembly Fund, Petroleum Fund, and HIPC/MDRI outlays. \\
3/ Wages and salaries, transfers, interest, and foreign-financed and trust fund capital spending.
\end{tabular}

23. A medium-term fiscal strategy is needed, integrating the potential revenues from the oil and gas sector. The authorities provisionally plan to use oil incomes in 2011-12 to finance infrastructure projects; these investments would then be scaled back in subsequent years, to allow part of oil revenues to be saved in a "heritage fund" to support public programs as oil revenues decline. The mission supported a front-loaded investment strategy, given Ghana's infrastructure needs, while cautioning that spending should be based on cautious oil revenue assumptions and take into account the country's absorptive capacity. Firm control will be needed over recurrent spending, to ensure that it does not expand to fill the newly available fiscal space. Equally, oil receipts should not be at the expense of strengthened non-oil revenue mobilization; the latter will be needed if donor support were to decline following Ghana's move to oil producer status. The mission noted that these considerations should be reflected in a fiscal rule for managing oil revenues, a topic to be revisited after the June 2009 technical assistance mission on resource sector taxation. The mission welcomed the authorities intention to ensure that the oil accounts are fully and transparently integrated into the fiscal accounts, and the plan to extend Ghana's EITI participation to the oil sector (MEFP, $₫ 38) .{ }^{6}$

\section{The joint Bank-Fund external debt sustainability analysis (DSA) suggests that} Ghana's external debt dynamics remain subject to moderate risk of debt distress. This assessment is broadly unchanged from the 2008 DSA, though the baseline debt trajectory is more favorable, reflecting the incorporation into the baseline of the Ghana's projected move to oil producer status in 2011. Assuming successful fiscal consolidation and sustained robust growth in the non-mineral economy, net public debt is projected to decline by about 35 percentage points of GDP during 2010-2029, to about 37 percent of GDP (or slightly below the post-HIPC/MDRI debt-GDP ratio in 2006). Stress tests suggest that external debt

\footnotetext{
${ }^{6}$ EITI would also be extended to forestry and fishing.
} 
indicators remain generally resilient to less favorable outcomes, while sustainability of the overall net debt position depends critically on maintaining a strong primary fiscal position.

\section{Monetary, Exchange Rate, and Financial Sector Policies}

To stem the inflationary impact of currency depreciation and higher fuel prices, monetary policy may need to be tightened further. Banking risks have risen, and supervisors will need to be alert to emerging strains.

\section{Monetary policy}

\section{Discussions focused on the factors contributing to rising inflation under the IT} framework. The required elements of an IT regime are in place (Box 2). The mission noted, however, that IT peer countries were typically more aggressive in monetary tightening than Ghana during 2007-08. The authorities noted that inflation in these countries also exceeded target levels, owing to the global food and fuel price shocks. More crucially, in Ghana's case, monetary tightening was overwhelmed by highly expansionary fiscal policies. With an end to fiscal dominance, the Bank of Ghana views its tools for monetary management as adequate for controlling inflation. Based on evidence of tightening credit conditions and easing demand pressures, the central bank projects a decline in inflation to $14 \frac{1}{2} 2$ percent at end-2009, reaching single-digits in 2010 .

26. The case for further monetary tightening has strengthened since the mission. In May, staff saw little scope for near-term easing, given the modest level of real interest rates and the vulnerability of inflation expectations to further currency depreciation. Indeed, the mission cautioned that further tightening might be needed to achieve the central bank's disinflation goal. This argument has been subsequently reinforced by the 30 percent rise in domestic petroleum product prices in early-June, based on rising global oil prices. Further tightening would help resist second-round inflationary pressures. The authorities indicated a readiness to consider a tighter monetary stance in the July MPC meeting, should conditions warrant. 


\section{Box 2: Ghana's Inflation-Targeting Framework}

The Bank of Ghana (BoG) formally launched its inflation targeting (IT) regime in 2007, and has benefited from advice from, among others, the Bank of England and Fund staff. The framework targets the 12-month change in the headline CPI, and the BoG also monitors a number of core inflation measures.

The key institutional arrangements for an IT framework are in place, including central bank policy independence, instrument independence, bi-monthly meetings of a Monetary Policy Committee (MPC), and generally good transparency.

BoG staff produce a range of inflation forecasts for the MPC, using a range of macro models (from simple autoregressive to relatively up-to-date multiple-equation time series models); they also track surveys of inflation expectations. Efforts continue to strengthen the models-for example, to identify a robust link between the fiscal stance and inflation. Real sector data are limited (no monthly production or quarterly GDP data), but the BoG has developed a quarterly composite indicator that tracks GDP trends moderately well (paragraph 13).

The BoG has the operational tools to influence inflationary pressures through credit market conditions. The commercial bank lending rate has closely tracked the BoG prime rate and, more recently, has responded to conditions in the interbank and t-bill markets, which the BoG influences through open market operations.

In the view of staff, the next developmental phase for Ghana's IT regime is to gain greater comfort in basing policies on the available analytical tools. For example, in February 2009, the BoG's quarterly macro model suggested that interest rates would need to rise significantly by the end of the second quarter to counter inflationary expectations. In practice, the policy rate was increased 150 basis points in February, and not subsequently (Figure below).

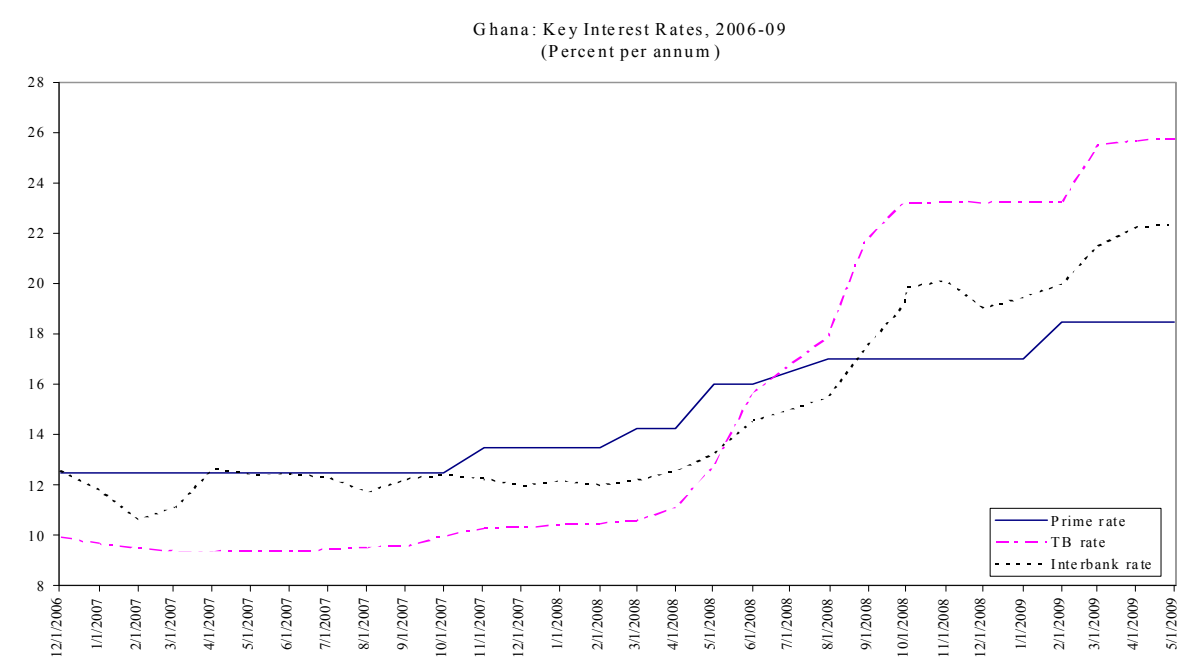

\section{To enhance the IT framework, the authorities intend to strengthen}

communication (MEFP, ๆ58). The mission suggested that greater clarity was needed about the monetary transmission mechanism and Bank of Ghana's policy stance. The authorities 
concurred with staff that a renewed emphasis on communications was needed, including through revamping the central bank website and clarifying its preferred measure of core inflation. The authorities disagreed that more frequent Monetary Policy Committee meetings would be helpful, noting that data limitations preclude a substantial reassessment of the policy stance more often than bi-monthly.

\section{Exchange rate policy}

\section{The authorities intend to maintain a flexible exchange rate regime consistent} with the inflation-targeting framework (MEFP, $\mid 59$ ). They envisage that policy tightening, combined with new external funding, will help stabilize the currency, a necessary condition for successful disinflation. It was agreed that the Bank of Ghana should refrain from intervention in the foreign exchange market, other than through its regular weekly sales of foreign exchange to meet priority demands (e.g., debt service and oil import costs). Consistent with Ghana's low official reserve cover, the authorities intend to use the possible new SDR allocation to rebuild foreign exchange reserves (MEFP, \28). Looking further ahead, the mission suggested that the authorities should aim to develop the role of the private sector in the foreign exchange market to handle the increased turnover that will emerge with the start of oil production.

\section{The recent currency depreciation may have partly corrected for earlier}

overvaluation. Staff analysis suggests that the real effective exchange rate in the first quarter of 2009 may have remained modestly overvalued (Box 3). In the event of further currency pressure, the mission urged tight monetary conditions to limit any inflationary pass-through. The authorities noted that the staff's currency valuation analysis produced mixed results, including some that suggested undervaluation. They interpreted these findings as consistent with their view that the currency was broadly appropriately valued.

\section{Financial sector issues}

\section{Discussions focused on the impact of the global crisis on Ghana's financial} system and the prospective risks. An in-depth assessment by Fund staff was generally positive about supervisory capacity in Ghana, but noted that banking sector risks were rising (Box 4). The mission discussed how to limit spillovers from the global financial crisis and how to strengthen governance and board oversight in state-owned banks. The authorities indicated that a program to address these risks was being rolled out (MEFP, $\mid 61$ ). 


\section{Box 3. Ghana: Exchange Rate Assessment}

As noted in paragraph 12, the large depreciation of the cedi-dollar exchange rate since mid-2008 was offset, in large part, by high inflation and the strength of the dollar. With Ghana's real effective exchange rate in early 2009 remaining stronger than in 2003-04 (figure below), a currency valuation exercise was conducted to assess whether further real effective adjustment is warranted.

Analytical approaches based on developments in the external current account tend to suggest currency overvaluation. The underlying current account deficit after eliminating temporary influences is estimated at 11.5 percent of GDP in 2008, well above the norm of 8.7 percent of GDP derived through the macro-balance approach. It is also larger than the net foreign liabilitystabilizing current account deficit of 9.8 percent of GDP implied by the external sustainability approach. To reduce the external deficit to the latter range, a real effective depreciation of 7-12 percent would be needed.

Approaches that model the real exchange rate directly suggest potential currency undervaluation. Econometric analysis drawing on Ghanaian and cross-country data suggests that the recent currency appreciation can be explained by Ghana's faster output growth (Balassa-Samuelson effects) and positive terms of trade changes. These econometric approaches suggest that the real effective exchange rate could be undervalued by 31-39 percent.

Fund staff tend to discount these econometric findings, given the fragility of the estimation results. In particular, the findings are very sensitive to the estimation time period and the explanatory variables included. Until more robust estimates can be developed, more weight has been given to signs of possible modest overvaluation coming from Ghana's widening current account deficit.

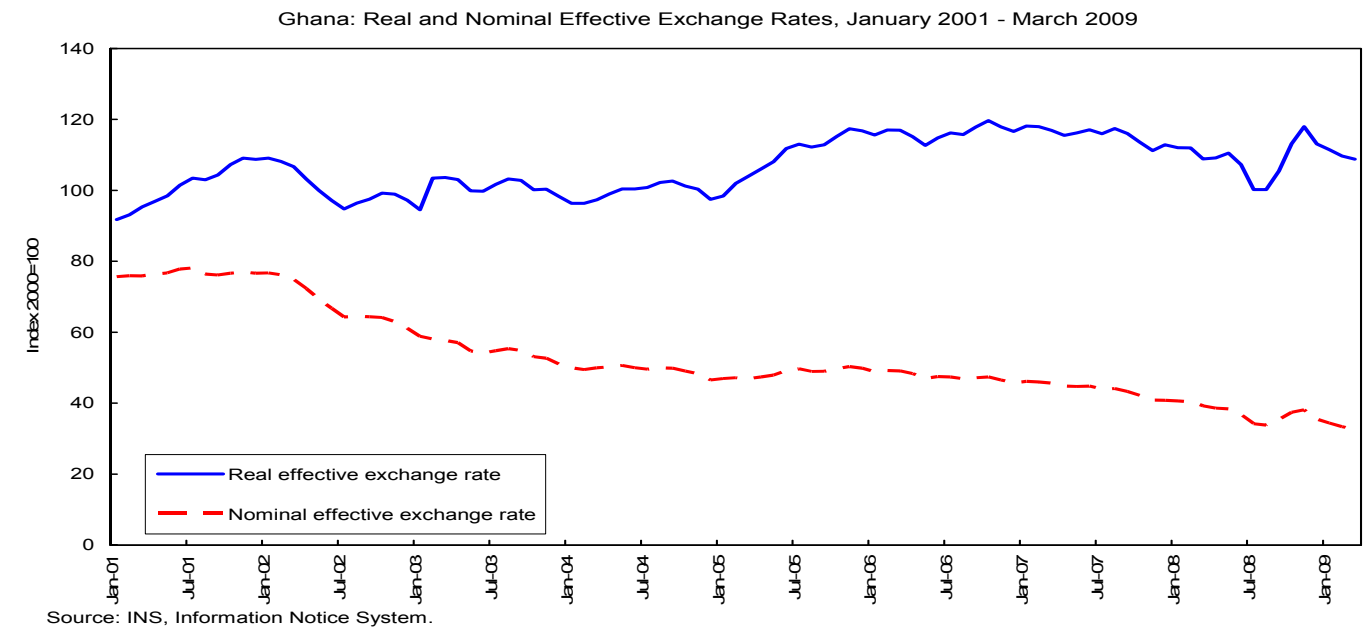




\section{Box 4. Challenges for Ghana's Financial System}

The direct impact of the global financial crisis on Ghana's financial system has been relatively limited. This reflects Ghanaian banks' limited integration with global markets and their reliance on domestic funding. Credit markets remain buoyant, and financial institutions are generally stable. In the debt markets, some foreign investors have exited from government securities, spreads on the international bond have increased, and options for new sovereign issues have narrowed. Domestic inflationary pressures and declining foreign inflows have contributed to higher interest rates in domestic money markets and pressures in the foreign exchange market.

Strong banking sector expansion has created vulnerabilities. Despite strengthened supervision, an environment of rapid financial growth, intense competition, and rising funding costs has encouraged risk taking. Banks' loan portfolios are often concentrated on a few large borrowers or sectors; some foreign currency loans are to unhedged borrowers; and rapid growth of domestic branch networks against a background of rapidly expanding cross border operations by parent banks has increased potential for strategic and operational risk. Some banks' internal controls and risk management practices have been stretched. The ratio of non-performing loans have risen to a year high of 9.6 percent at end-March 2009, with above average NPLs in the forestry, mining, and construction sectors (see below); a couple of banks have also incurred substantial losses from their expansion strategy. Weaknesses in corporate governance and board oversight are also evident in some of the stateowned banks, with one recently experiencing apparent high-level fraud. Banks have begun to limit their risk exposure, tightening lending standards. But this may not be enough to avoid a further deterioration in loan books.

Ghana: Sectoral NPLs

(In percent of total sectoral loans)

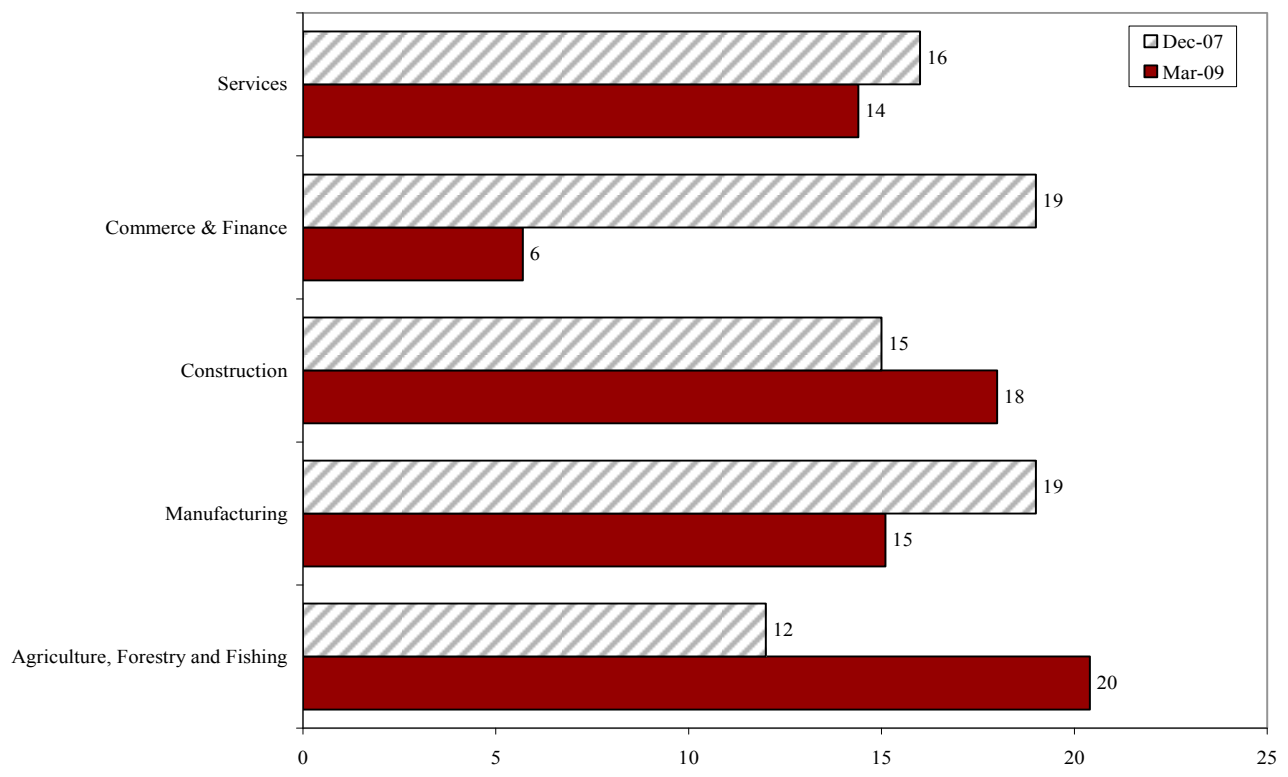

Gaps in cross border supervision and in the financial infrastructure add to the risks. Some of the home supervisors of parent banks do not undertake consolidated supervision, thus there is no oversight of group risks. In some cases, parent banks are registered in jurisdictions where supervisory oversight is weak, prudential regulations lax and banking systems are exhibiting some stress. Though progress has been made in improving the environment for creditor rights, banks continue to experience difficulties in foreclosing on collateral. Also, credit risk assessment of borrowers is made difficult by weaknesses in accounting and auditing practices in SMEs and the credit registry is just being established.

The Bank of Ghana has a well established framework that enables it to monitor risks in the financial system and to respond to financial system stress in a timely manner. These include an established off-site framework for monitoring risks supported by a well-developed stress testing framework. A Lender of Last Resort facility is available, and the BoG has the legal authority to facilitate prompt corrective actions. Notwithstanding this, the operational framework for providing liquidity support is limited in scope, both in the tenors of the loans that can be advanced and in the range of acceptable securities, which could prove limiting under lengthy adverse conditions. There are also no deposit insurance or contingency cross-border crisis arrangements. 


\section{E. Other Issues}

31. National accounts statistics. The mission discussed the outcome of a recent Fund review of Ghana's national accounts rebasing exercise, which suggests that economic activity has been significantly under-estimated, reflecting outdated methodology and lapses in incorporating new source data. Upward revisions could be as large as 50 percent, paralleling revisions observed in other countries' rebasing exercises (see Figure 1).

\section{The NeW PRGF ArRangement}

\section{A. Program Objectives and Design}

32. The PRGF-supported program builds on Ghana's second Poverty Reduction Strategy (GPRS II). ${ }^{7}$ The arrangement will cover the three-year period from Board approval. Proposed access is 105 percent of quota (SDR 387.45 million), somewhat above the norm for a fifthtime PRGF-user (70 percent of quota), reflecting program strength and balance of payments need. The arrangement has eight disbursements (Table 8), with some front loading into 2009-10 when the adjustment effort and balance of payments need are strongest.

33. The main goal of the program is to eliminate Ghana's large fiscal imbalances by 2011 and put in place strengthened institutions for public financial management (PFM). Restoring macroeconomic stability will eliminate the most pressing obstacle to continued strong growth and poverty reduction. At the same time, fiscal consolidation will create the necessary room for maneuver, so that when oil revenues come on stream in 2011 , they can be dedicated to new growth-promoting and poverty-reducing investments that benefit future generations, rather than being diverted to unproductive recurrent spending. To support macroeconomic stabilization, the inflation targeting regime will be further strengthened, notably in the critical area of communications and transparency. Policy transparency would be underpinned by extending the Extractive Industry Transparency Initiative (EITI) to the oil sector (MEFP, ๆ38), and by expanded publication of fiscal data (MEFP, ๆ67).

\section{B. Program Monitoring}

34. The program will be initially monitored on a quarterly basis, given the near-term risks from the global recession. The first two reviews would assess developments relative to performance criteria for end-September and end-December 2009. Subsequently, program monitoring would move to a semi-annual basis, with tests dates for end-June and endDecember through 2011, and a final review based on a test date of end-March 2012. Quantitative targets would be as described below and in MEFP Table 1.

\footnotetext{
${ }^{7}$ Work to develop an updated GPRS III to cover the period starting 2010 has been initiated (MEFP, $\mid$ |56). A status report will be provided at the time of the first review.
} 
- $\quad$ Fiscal performance would be assessed by the overall balance including grants. Given the need for spending restraint, adjusters would limit the scope for new spending commitments in response to higher-than-programmed grant or loan funding. Shortfalls in financing would be partially covered by spending cuts, and partly by higher resort to domestic financing, up to a ceiling (TMU, $\mid 22$ ).

- Debt sustainability would be promoted by limiting nonconcessional financing to high-return infrastructure and energy projects. The program would provide for guarantees for Ghana National Petroleum Company borrowing for oil and gas investments of up to US\$300 million during 2009-10 (MEFP, \54).

- Monetary policy will be subject to review clauses linked to inflation ranges around the target disinflation path. To underpin the IT framework, net domestic assets of the Bank of Ghana will be an indicative target.

\section{Structural Conditionality}

35. Structural conditionality focuses on strengthening fiscal institutions (tax policy, revenue administration, PFM) and addressing recurrent sources of spending pressure (particularly energy subsidies and the public sector wage bill) (MEFP, Table 2).

36. Tax policy. Ghana has the essential pillars of a modern tax system, but two features erode revenue potential and hinder economic growth. The system is overwhelmed by special, often discretionary, exemptions that undermine confidence in the equity and effectiveness of the tax regime. Thresholds for VAT and business taxation are also too low, leading to wasteful use of scarce administrative resources on small taxpayers. Steps taken in June 2009 to rein in exemptions will be intensified in the 2010 budget (MEFP, $\mid 24, \boldsymbol{q} 32$ ). To simplify VAT administration, a review will also be conducted this year to reduce the number of zerorated VAT items and convert domestic zero-rating to standard rate taxation (MEFP, $\mid 32$ ). VAT thresholds and small business taxation will also be revisited (MEFP, $\uparrow 33$ ).

37. Revenue administration. Ghana, formerly in the vanguard of revenue administration reform, has lagged behind best practice in recent years. In particular, international experience has shown the circa-1980s model of a semi-autonomous revenue service under a governing board to be less effective than the single revenue authority model, under which customs and tax administration are managed by a single executive and benefit from information and other synergies. Further, the separate agencies for income tax and VAT administrations have proven inefficient and costly, and therefore Ghana would benefit from the experience of most other countries by moving towards a single integrated domestic tax agency within the intended revenue authority. The authorities intend to launch a modernization strategy with these goals by end-2009 (MEFP, $\uparrow 35$ ).

38. Public expenditure management. Roll out of Ghana's integrated financial management information system (IFMIS) has stalled at the pilot stage, and institutional 
capacity for tracking and controlling spending has been weak, giving rise to reoccurring domestic arrears. The government intends to review by end-2009 the shortcomings of the existing IFMIS system, and develop a plan for its wider adoption or for a move to an alternative system (MEFP, $\{42$ ). In the interim, intensified monitoring, supported by strengthened inter-agency coordination, is being adopted to manage the budget through 2009 (MEFP, $\llbracket 40-41$ ). The authorities are also taking stock of the outstanding claims from 2008, and will adopt policies to avoid new arrears in the future (MEFP, $\mid 53$ ).

39. Public sector reform. A comprehensive public sector modernization program is needed, addressing the size, effectiveness, and remuneration system of the public service. The World Bank will support this reform program. In the near-term, the Fund program aims at strengthening monitoring and control of the payroll numbers through comprehensive computerization of staffing data, centralized hiring procedures, payroll audits, and the launch of a rationalization process for public agencies (MEFP, $\uparrow 45-49$ ).

40. Energy sector reforms. In the past, under-pricing of electricity supplies and petroleum products has required government subsidies, undermined the financial accountability of the state-owned enterprises, and left a legacy of cross-debts and arrears. The World Bank is supporting a financial restructuring and recovery program for the sector. The PRGF is designed to ensure the adoption and maintenance of cost-recovery pricing (MEFP, \52). A key condition will be the adjustment of electricity to cover operating costs from end-2009, with cost-recovery maintained through subsequent quarterly reviews.

\section{Program Financing}

\section{Even with policy adjustment, Ghana faces balance of payments financing gaps of US\$450 million in 2009 and \$325 million in 2010.}

- $\quad$ The fiscal financing gap is projected at US\$250 million in 2009, declining to half that size in 2010. The majority of the gap would be closed in 2009 through a new World Bank Development Policy Loan, which would augment its 2009 funding pipeline by US\$200 million. Ghana is a candidate for a new vulnerability facility being developed by the European Commission, which could close up to half of the remaining gap. The authorities have approached other bilateral donors, including the African Development Bank, and expect to close most, if not all, of any remaining gap for 2009. Donors have noted that while additional financing for 2009 has been difficult to mobilize in the context of established multi-year support agreements, the PRGF arrangement would provide the macroeconomic assurances necessary to maintain existing support levels as programs come up for review. The authorities have indicated that any residual financing shortfall would be met through a combination of further spending restraint and some limited additional use of domestic financing (TMU, $\mathbb{q} 22$ ). 
Further balance of payments financing of \$200 million in 2009 and 2010 would be provided under the PRGF arrangement. This would stabilize gross reserve levels at 2 months import cover, a level that would rise to $2 \frac{1}{2}$ months' cover with the proposed new SDR allocation. The balance of payments financing need assumes that private capital inflows remain low through 2009-10, except for oil sector investments in imported rig equipment. Net private remittances are also projected to decline 30 percent in 2009, with limited recovery before 2011.

\section{E. Program Risks and Mitigation}

42. Program monitoring should be vigilant. The authorities will need to monitor activity and revenue collections closely to ensure that contingency plans for spending restraint can be activated, if needed, to safeguard the fiscal position.

43. Strong communications will also be key. Adjustments to utility pricing to achieve the fiscal deficit target for 2010 will be an important challenge, especially if global oil prices continue to rise. To support this core element of the program, public outreach will be important. Looking further ahead, it will be important to convince interest groups that the momentum of reforms should be sustained after the start of oil production. Although oil revenues will bring the 2011 deficit target within reach, this should not be at the expense of revenue mobilization in the non-oil economy and should not delay steps to rationalize public administration. Without these continuing reforms, recurrent spending programs could expand to absorb the new fiscal space created by Ghana's oil wealth.

\section{Ghana has adequate capacity to meet its financial obligations to the Fund. The} country has an established record of timely servicing its obligations to the Fund, and the DSA places Ghana at a moderate risk of debt distress (paragraph 24). Debt service to the Fund would remain modest in absolute terms, and below 1 percent of exports of goods and services by the end of the program period.

\section{Staff Appraisal}

45. Ghana's current challenges reflect economic mismanagement, exacerbated by global factors. The pursuit of highly expansionary fiscal policies through 2008 despite growing signs of macroeconomic imbalance contributed to high rates of inflation, rising interest rates, and currency pressures. Macroeconomic correction has been complicated by the global financial crisis, which closed off global market funding options and required resort to exceptional financing from development partners.

\section{The external environment remains more favorable than for many countries.}

Ghana's terms of trade have strengthened during the global recession, supporting incomes and growth, while the domestic financial system has proved to be relatively insulated from the global financial storm. Given this, while projections are more than usually uncertain, growth is projected to exceed the global average through 2009-10. There are important risks, 
however, notably to private sector remittances and foreign direct investment. Equally, the terms of trade benefit has declined with the recovery in oil prices. If private sector activity slows sharply, the challenges for macroeconomic management would be complicated.

47. Fiscal consolidation will be central to restoring macroeconomic stability. The deficit target for 2009 is appropriately ambitious, and the focus on expenditure restraint is welcome. Contingency plans for further expenditure tightening will be important to keep the deficit on track in the event of revenue shortfalls. Further fiscal measures will be needed to reduce the deficit to 6 percent of GDP in 2010. An increase in utility tariffs will be needed to cover the rising costs of power generation. Steps to control public wage growth should be pursued, along with options for strengthened revenue mobilization. Even after a significant fiscal consolidation, however, Ghana's medium-term prospects remain subject to important challenges, including the need to reduce public sector debt to more sustainable levels.

\section{Revenues from the start of oil production in 2011 will create important new}

fiscal space. Part should be used to further reduce the fiscal deficit and accumulate assets for the post-oil period, while part could finance an expansion of infrastructure spending after two relatively tight years in 2009-10. However, oil revenues will be modest relative to many producers, and the production period is expected to be relatively short. Given this, every effort should be made to avoid complacency about the fiscal outlook from 2011. Continuing efforts will be needed to strengthen domestic revenue mobilization, including to safeguard against a potential phasing out of donor grants, and expenditure policies should continue to seek improved value for money in public services. Structural fiscal reforms will be critical to enhance fiscal management, including in the rapidly-approaching oil era.

\section{With tighter fiscal policies, the onus for inflation control now falls squarely on} the IT framework. With rising global oil prices and an associated large increase in domestic petroleum product prices, the case for further monetary policy tightening is strong. To reinforce the IT framework, clearer communications are needed about the policy framework and monetary stance.

50. Despite currency depreciation, the real effective exchange rate remains relatively appreciated. With some risks that the currency remains slightly overvalued, continued exchange rate flexibility will be important.

\section{Risks in the banking system will require careful monitoring. Surveillance should} be strengthened, and commercial banks encouraged to reinforce risk management and corporate governance practices. Gaps in cross border supervision require stepped up regional collaboration. Domestically, enforcement of creditor rights should be further strengthened, and further momentum given to the operations of the credit reference bureau. Staff welcomes the authorities' interest in an FSAP update.

\section{Statistical data should be further strengthened for surveillance purposes.}

Improved data on the national accounts are a priority. 
53. Staff supports the authorities' request for a new PRGF arrangement. The focus of the new program on fiscal consolidation and supporting structural fiscal reforms is appropriate. Progress is needed on a range of fronts to regain a vanguard position in public finance management. Reforms are likely to be resisted by pressure groups, particularly as oil production approaches. However, without reform, Ghana risks dissipating its oil revenues with little long-term benefit for growth and poverty reduction. An active communication strategy will be critical to sustaining the momentum of reform.

54. It is proposed that the next Article IV consultation with Ghana take place within 24 months, in accordance with the decision on consultation cycles in program countries. 
Figure 1. Possible Impact of National Accounts Revisions (assuming a 50 percent upward revision to real and nominal GDP) 1/
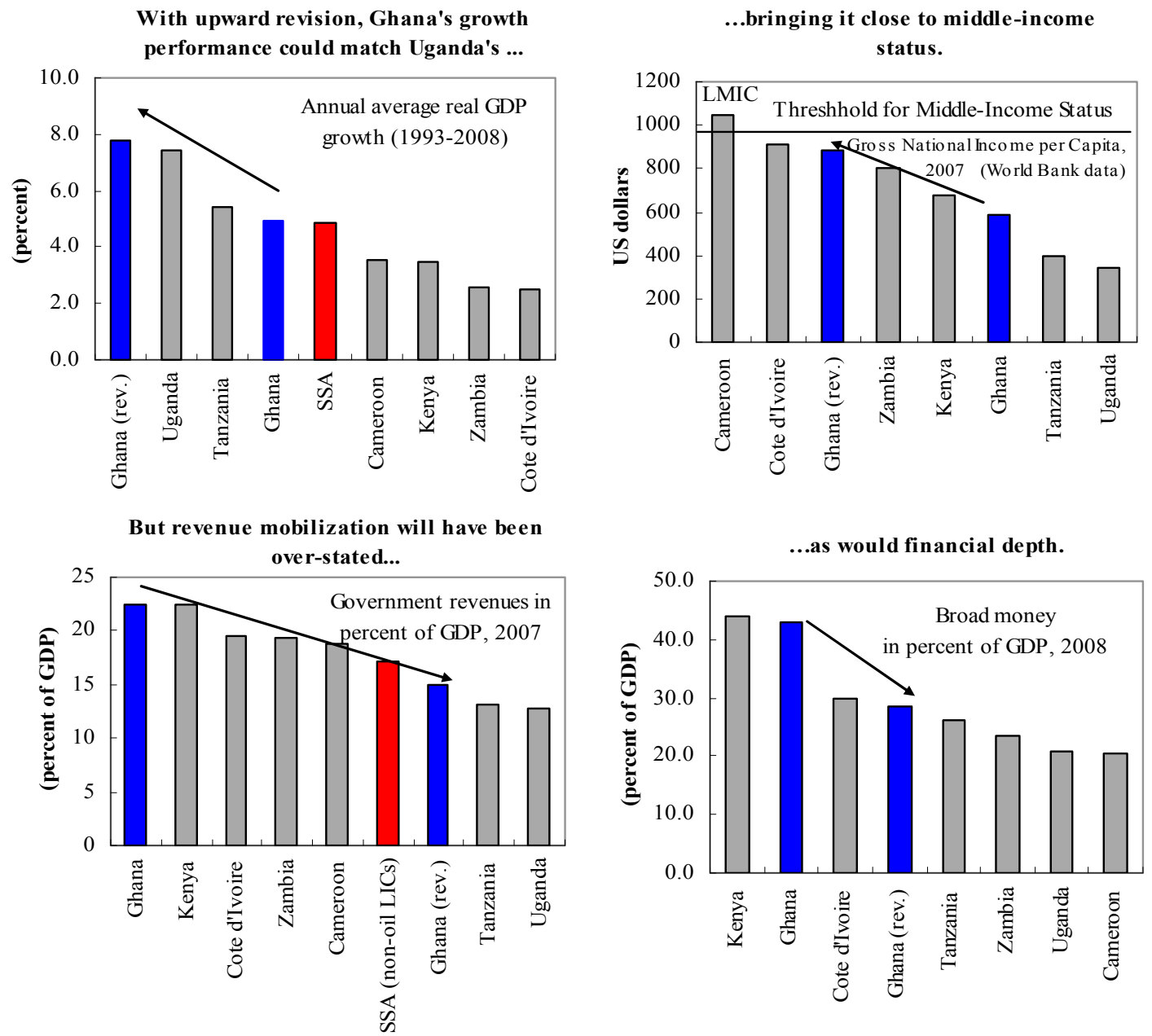

Fiscal imbalances would remain important, with a high public sector wage bill...
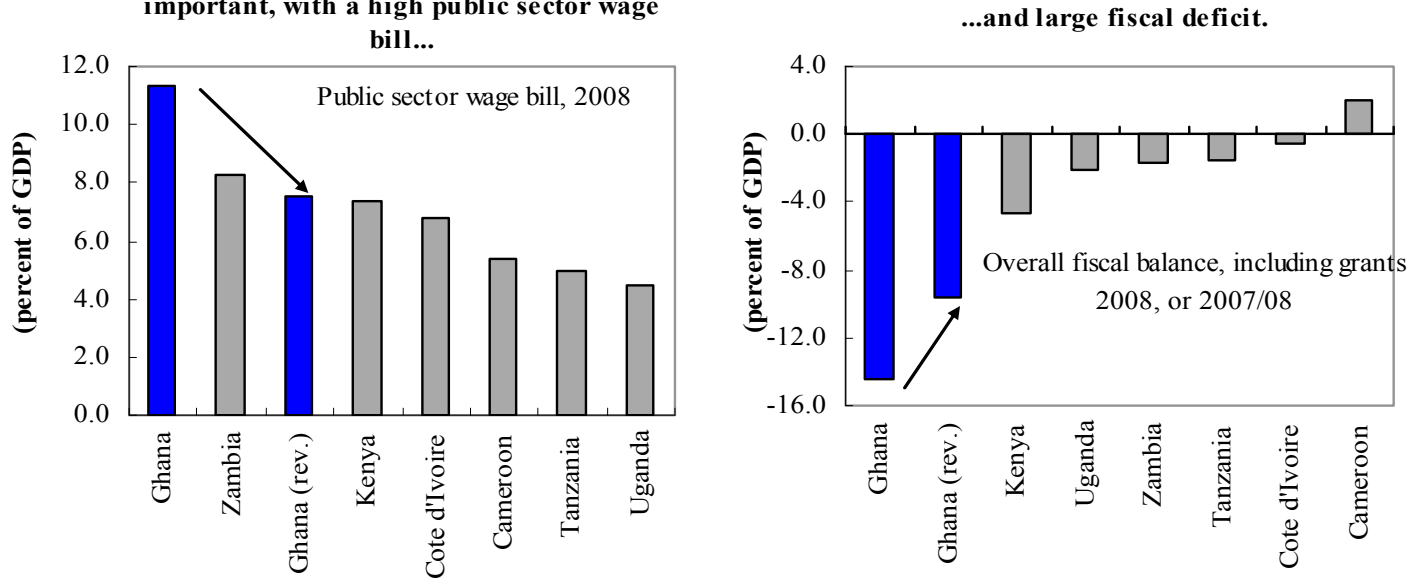

Source: Country authorities, and Fund staff estimates.

1/ The assumed 50 percent upward revision is consistent with preliminary back-of-the-envelope calculations, based on new source data. Update accounts will be available in late-2009 or early-2010. 
Figure 2. Ghana: Business and Financial Indicators

Ghana's business climate is favorable ...

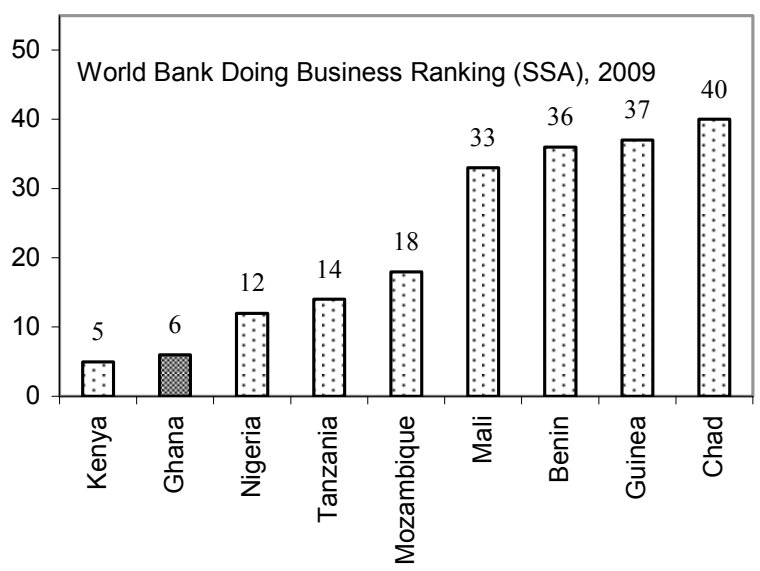

Oil production from 2011 will boost incomes...

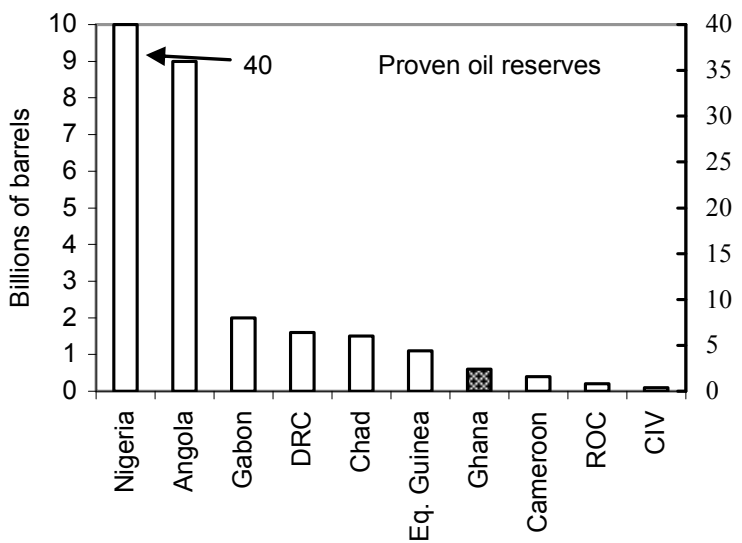

Banking indicators have begun to deteriorate ...

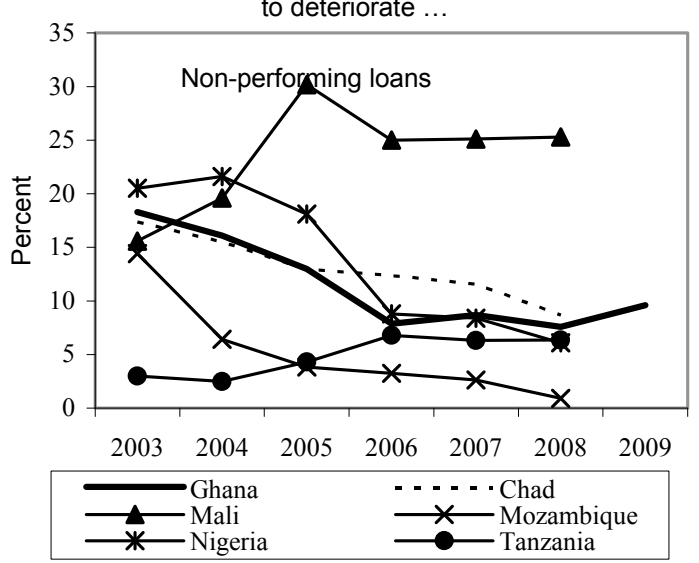

... and governance is strong.

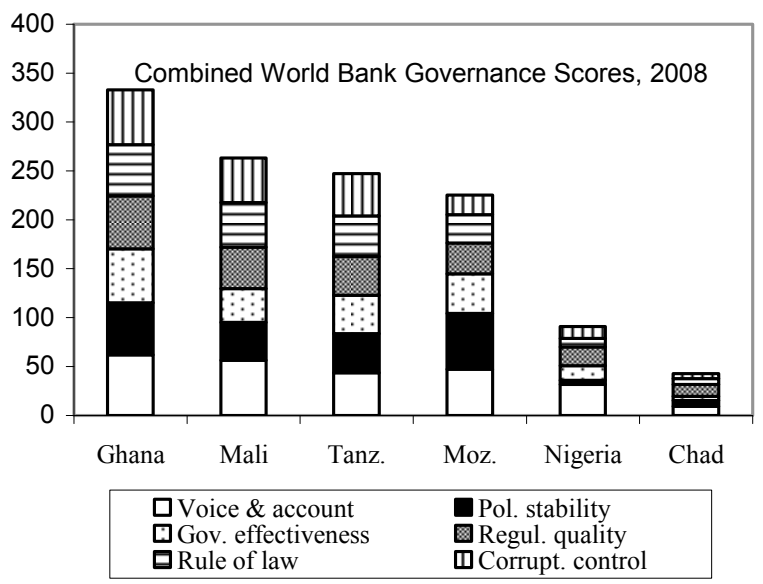

... but a more affordable public sector will remain a priority.

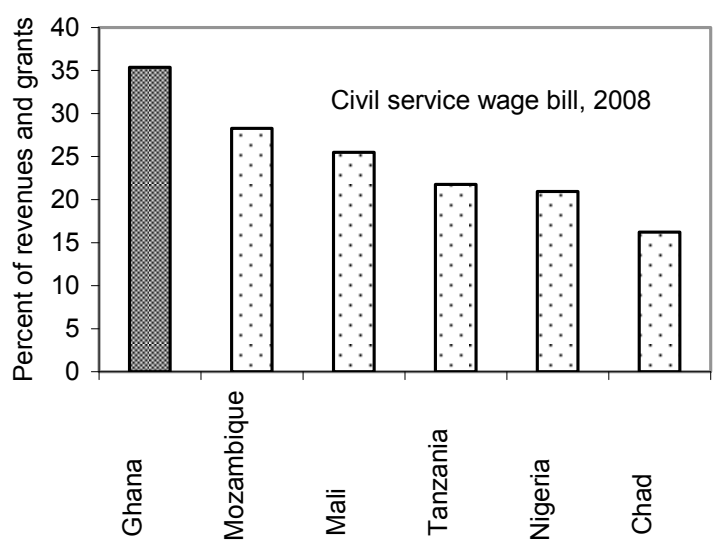

... and inflation control remains a problem.

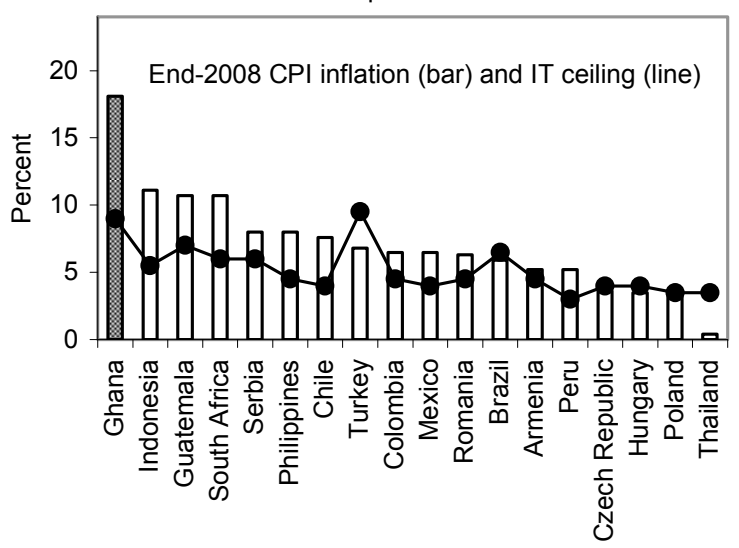

Sources: U.S. Energy Information Administration, OPEC, Annual Statistical Bulletin,2007, and BP Statistical Review of World Energy 2008; World Bank, Cost of Doing Business, 2009, and Governance Indicators, 2008; central bank websites; and IMF Staff calculations and projections. 
Table 1. Ghana: Selected Economic and Financial Indicators, 2006-14

\begin{tabular}{|c|c|c|c|c|c|c|c|c|c|}
\hline & 2006 & $\begin{array}{c}2007 \\
\text { Est. }\end{array}$ & $\begin{array}{c}2008 \\
\text { Est. }\end{array}$ & $\begin{array}{l}2009 \\
\text { Proj. }\end{array}$ & $\begin{array}{l}2010 \\
\text { Proj. }\end{array}$ & $\begin{array}{l}2011 \\
\text { Proj. }\end{array}$ & $\begin{array}{l}2012 \\
\text { Proj. }\end{array}$ & $\begin{array}{l}2013 \\
\text { Proj. }\end{array}$ & $\begin{array}{l}2014 \\
\text { Proj. }\end{array}$ \\
\hline & \multicolumn{9}{|c|}{ (Annual percentage change; unless otherwise specified) } \\
\hline \multicolumn{10}{|l|}{ National accounts and prices } \\
\hline Real GDP & 6.4 & 5.7 & 7.3 & 4.5 & 5.0 & 24.2 & 7.1 & 5.3 & 5.0 \\
\hline Real GDP (nonoil) & 6.4 & 5.7 & 7.3 & 4.5 & 5.0 & 6.2 & 6.2 & 6.2 & 5.9 \\
\hline Real GDP per capita & 3.8 & 3.0 & 4.6 & 1.9 & 2.4 & 21.1 & 4.5 & 2.6 & 2.4 \\
\hline GDP deflator & 12.8 & 13.9 & 16.9 & 17.2 & 11.0 & 6.7 & 3.1 & 4.6 & 5.5 \\
\hline Consumer price index (annual average) & 10.2 & 10.7 & 16.5 & 18.2 & 10.5 & 8.4 & 4.5 & 5.0 & 5.0 \\
\hline Consumer price index (end of period) & 10.9 & 12.7 & 18.1 & 14.6 & 9.2 & 8.0 & 5.0 & 5.0 & 5.0 \\
\hline \multicolumn{10}{|l|}{ External sector } \\
\hline Exports, f.o.b. (percentage change, in US\$) & 33.0 & 11.9 & 26.3 & 9.8 & 5.9 & 51.1 & 7.6 & 4.9 & 5.6 \\
\hline Excluding oil exports & 33.0 & 11.9 & 26.3 & 9.8 & 5.9 & 3.4 & 3.9 & 6.6 & 7.5 \\
\hline Imports, f.o.b. (percentage change, in US\$) & 26.3 & 19.4 & 27.3 & -9.4 & 8.5 & 14.0 & 4.9 & 8.0 & 1.7 \\
\hline Export volume (excluding oil) & 15.4 & -3.7 & 7.5 & 7.2 & 7.3 & 9.8 & 6.8 & 7.0 & 5.9 \\
\hline Import volume & 14.4 & 14.6 & 14.1 & 6.4 & 2.0 & 12.1 & 3.8 & 7.2 & 1.4 \\
\hline Terms of trade & 4.4 & 11.5 & 5.3 & 20.3 & -7.3 & -7.4 & -3.8 & -1.2 & 1.2 \\
\hline Nominal effective exchange rate (end of period) & -8.2 & -10.8 & -13.1 & $\ldots$ & $\ldots$ & $\ldots$ & $\ldots$ & $\ldots$ & $\ldots$ \\
\hline Real effective exchange rate (end of period), (depreciation -) & 1.2 & 1.7 & 2.6 & $\ldots$ & $\ldots$ & $\ldots$ & $\ldots$ & $\ldots$ & $\ldots$ \\
\hline Cedis (new) per U.S. dollar (end of period) & 0.92 & 0.97 & 1.27 & $\cdots$ & $\cdots$ & $\cdots$ & $\cdots$ & $\cdots$ & \\
\hline \multicolumn{10}{|l|}{ Money and credit } \\
\hline Net domestic assets ${ }^{1}$ & 13.4 & 32.6 & 38.6 & 8.6 & 7.0 & 2.8 & -1.9 & -2.1 & 0.6 \\
\hline Real private sector credit (\% annual changes) & 28.6 & 41.9 & 27.3 & 9.0 & 15.0 & 10.0 & 10.0 & 10.0 & 10.0 \\
\hline Broad money (including foreign currency deposits) & 38.8 & 35.9 & 40.2 & 21.7 & 16.9 & 27.9 & 18.6 & 14.8 & 15.4 \\
\hline Velocity (GDP/average broad money) & 3.5 & 3.0 & 2.8 & 3.0 & 3.0 & 3.2 & 3.0 & 2.8 & 2.7 \\
\hline Prime rate (Bank of Ghana; percent; end of period) & 12.5 & 13.5 & 17.0 & $\ldots$ & $\ldots$ & $\ldots$ & $\ldots$ & $\ldots$ & \\
\hline & \multicolumn{9}{|c|}{ (Percent of GDP) } \\
\hline \multicolumn{10}{|l|}{ Investment and saving } \\
\hline Gross investment & 30.4 & 33.8 & 36.2 & 30.6 & 37.3 & 37.4 & 34.9 & 36.1 & 32.8 \\
\hline Private $^{2}$ & 18.0 & 19.5 & 20.5 & 18.0 & 25.4 & 24.5 & 22.4 & 23.4 & 20.0 \\
\hline Central government ${ }^{3}$ & 12.4 & 14.4 & 15.7 & 12.6 & 11.9 & 12.9 & 12.5 & 12.8 & 12.8 \\
\hline Gross national saving & 20.5 & 21.8 & 16.9 & 17.6 & 21.4 & 27.9 & 26.6 & 26.6 & 26.8 \\
\hline Private $^{2}$ & 12.6 & 14.0 & 12.1 & 12.9 & 9.4 & 11.9 & 10.2 & 9.4 & 9.8 \\
\hline Central government $^{3}$ & 7.8 & 7.7 & 4.8 & 7.2 & 8.5 & 12.9 & 13.4 & 14.1 & 13.9 \\
\hline New fiscal measures (net S/l impact) & $\ldots$ & $\ldots$ & $\ldots$ & 0.0 & 3.5 & 3.1 & 3.1 & 3.1 & 3.1 \\
\hline Foreign savings & 9.9 & 12.0 & 19.3 & 13.0 & 15.9 & 9.5 & 8.3 & 9.5 & 6.0 \\
\hline & \multicolumn{9}{|c|}{ (Percent of nonoil GDP) } \\
\hline \multicolumn{10}{|l|}{ Government operations } \\
\hline Total revenue & 21.9 & 22.7 & 22.8 & 23.9 & 24.3 & 30.5 & 31.1 & 30.7 & 30.0 \\
\hline Grants & 5.4 & 6.1 & 4.7 & 6.0 & 5.0 & 3.9 & 4.0 & 3.8 & 3.8 \\
\hline Total expenditure & 34.4 & 37.3 & 41.0 & 36.7 & 37.9 & 41.0 & 40.3 & 39.0 & 38.5 \\
\hline New fiscal measures & $\ldots$ & $\ldots$ & $\ldots$ & 0.0 & 3.5 & 3.1 & 3.1 & 3.1 & 3.1 \\
\hline Overall balance (including grants) & -7.5 & -9.2 & -14.5 & -9.4 & -6.0 & -4.5 & -2.8 & -2.1 & -2.1 \\
\hline Net domestic financing & 5.0 & 1.3 & 9.8 & 4.8 & 0.8 & 0.2 & -0.8 & -0.8 & 0.3 \\
\hline Gross government debt & 42.0 & 51.2 & 58.2 & 66.4 & 66.6 & 66.0 & 63.7 & 64.7 & 64.9 \\
\hline Domestic debt & 24.8 & 26.4 & 29.0 & 28.5 & 24.9 & 22.1 & 19.6 & 20.6 & 21.9 \\
\hline External debt ${ }^{4}$ & 17.2 & 24.8 & 29.2 & 37.9 & 41.7 & 43.9 & 44.1 & 44.0 & 43.0 \\
\hline Net government debt (net of oil fund) & 42.0 & 51.2 & 58.2 & 66.4 & 66.6 & 66.0 & 63.7 & 60.8 & 58.1 \\
\hline & \multicolumn{9}{|c|}{ (Percent of GDP; unless otherwise specified) } \\
\hline \multicolumn{10}{|l|}{ External sector } \\
\hline Current account balance (including official transfers) & -9.9 & -12.0 & -19.3 & -13.0 & -15.9 & -9.5 & -8.3 & -9.5 & -6.0 \\
\hline Current account balance (excluding official transfers) & -13.0 & -15.8 & -23.5 & -18.0 & -19.9 & -11.9 & -10.8 & -11.9 & -8.5 \\
\hline NPV of external debt outstanding & $\ldots$ & 19.8 & 20.9 & 27.9 & 30.9 & 27.8 & 27.9 & 28.3 & 28.1 \\
\hline percent of exports of goods and services & $\ldots$ & 49.2 & 47.6 & 53.4 & 56.8 & 44.9 & 44.8 & 46.7 & 47.5 \\
\hline Gross international reserves (millions of US $\$)^{5}$ & 2,270 & 2,837 & 2,036 & 1,950 & 2,350 & 3,300 & 4,395 & 5,299 & 6,110 \\
\hline months of imports of goods and services & 2.7 & 2.7 & 2.2 & 1.9 & 2.0 & 2.7 & 3.4 & 4.0 & 4.3 \\
\hline Total donor support (millions of US\$) & 979 & 1,171 & 1,478 & 1,722 & 1,474 & 1,262 & 1,311 & 1,070 & 1,065 \\
\hline percent of GDP & 7.7 & 7.8 & 9.2 & 12.0 & 9.9 & 6.9 & 6.8 & 5.1 & 4.7 \\
\hline \multicolumn{10}{|l|}{ Memorandum items: } \\
\hline Nominal GDP (billions of GHc) & 11.672 & 14.0 & 17.6 & 21.6 & 25.1 & 33.3 & 36.8 & 40.5 & 44.9 \\
\hline
\end{tabular}

Sources: Data provided by Ghanaian authorities; and IMF staff estimates and projections.

${ }^{1}$ Percent of broad money at the beginning of the period

${ }^{2}$ Including public enterprises and errors and omissions.

${ }^{3}$ Before new fiscal measures.

${ }^{4}$ Includes potential new exceptional financing starting in 2009.

${ }^{5}$ Before possible new SDR allocation of US\$420 million in 2009. 
Table 2A. Ghana: Summary of Central Government Budgetary Operations, 2006-14 1/

\begin{tabular}{|c|c|c|c|c|c|c|c|c|c|c|}
\hline & \multirow[t]{2}{*}{2006} & \multirow[t]{2}{*}{2007} & \multirow{2}{*}{$\begin{array}{r}2008 \\
\text { Est. }\end{array}$} & \multicolumn{2}{|c|}{2009} & \multirow{2}{*}{$\begin{array}{c}2010 \\
\text { Proj. }\end{array}$} & \multirow{2}{*}{$\begin{array}{c}2011 \\
\text { Proj. }\end{array}$} & \multirow{2}{*}{$\begin{array}{c}2012 \\
\text { Proj. }\end{array}$} & \multirow{2}{*}{$\begin{array}{c}2013 \\
\text { Proj. }\end{array}$} & \multirow{2}{*}{$\begin{array}{r}2014 \\
\text { Proj. }\end{array}$} \\
\hline & & & & Budget & Art. IV & & & & & \\
\hline & \multicolumn{10}{|c|}{ (Percent of nonoil GDP, unless otherwise specified) } \\
\hline Total revenue and grants & 27.3 & 28.8 & 27.5 & 29.8 & 30.0 & 29.3 & 34.3 & 35.1 & 34.6 & 33.8 \\
\hline Revenue & 21.9 & 22.7 & 22.8 & 23.7 & 23.9 & 24.3 & 30.5 & 31.1 & 30.7 & 30.0 \\
\hline Tax revenue & 19.9 & 20.1 & 19.9 & 20.5 & 20.7 & 21.0 & 24.2 & 24.5 & 24.2 & 23.7 \\
\hline Direct taxes & 6.2 & 6.7 & 7.1 & 7.3 & 7.3 & 7.4 & 10.8 & 11.2 & 10.9 & 10.3 \\
\hline o/w Oil sector & $\ldots$ & $\ldots$ & $\ldots$ & $\ldots$ & $\ldots$ & $\ldots$ & 3.6 & 3.9 & 3.7 & 3.1 \\
\hline Indirect taxes & 9.1 & 9.3 & 8.7 & 9.0 & 9.0 & 9.1 & 8.8 & 8.8 & 8.8 & 8.8 \\
\hline Trade taxes & 4.6 & 4.1 & 4.1 & 4.3 & 4.5 & 4.6 & 4.6 & 4.6 & 4.6 & 4.6 \\
\hline Nontax revenue & 2.0 & 2.6 & 2.9 & 3.2 & 3.2 & 3.3 & 6.2 & 6.6 & 6.5 & 6.3 \\
\hline o/w Oil sector & $\ldots$ & $\ldots$ & $\ldots$ & $\ldots$ & $\ldots$ & $\ldots$ & 3.0 & 3.3 & 3.2 & 3.0 \\
\hline Grants & 5.4 & 6.1 & 4.7 & 6.1 & 6.0 & 5.0 & 3.9 & 4.0 & 3.8 & 3.8 \\
\hline Total expenditure & 34.4 & 37.3 & 41.0 & 36.6 & 36.7 & 37.9 & 41.0 & 40.3 & 39.0 & 38.5 \\
\hline Recurrent Non-interest expenditure & 18.6 & 19.8 & 21.5 & 19.9 & 19.8 & 22.1 & 22.4 & 22.5 & 22.1 & 21.5 \\
\hline Wages and salaries & 9.7 & 10.1 & 11.3 & 11.8 & 11.8 & 11.7 & 11.3 & 11.2 & 10.8 & 10.3 \\
\hline Goods and services & 3.7 & 4.0 & 3.7 & 1.9 & 1.9 & 1.9 & 2.0 & 2.2 & 2.2 & 2.3 \\
\hline Transfers & 2.5 & 4.1 & 3.5 & 4.2 & 4.3 & 4.2 & 4.2 & 4.2 & 4.0 & 4.0 \\
\hline Energy, utility and related subsidies & 1.8 & 0.2 & 1.5 & 0.3 & 0.3 & 3.2 & 3.6 & 3.8 & 3.9 & 3.7 \\
\hline Energy sector social safety net & 0.3 & 0.2 & 0.3 & 0.3 & 0.3 & 0.3 & 0.3 & 0.2 & 0.2 & 0.2 \\
\hline Utility company $\&$ refinery subsidies ${ }^{2}$ & 1.5 & 0.0 & 1.2 & 0.0 & 0.0 & 2.9 & 3.4 & 3.5 & 3.6 & 3.5 \\
\hline Reserve Fund & 0.8 & 1.4 & 1.6 & 1.6 & 1.6 & 1.2 & 1.2 & 1.2 & 1.2 & 1.2 \\
\hline Interest costs & 3.4 & 3.1 & 3.9 & 3.9 & 4.4 & 3.8 & 3.6 & 3.0 & 2.0 & 2.1 \\
\hline domestic & 2.6 & 2.3 & 2.7 & 2.4 & 2.9 & 2.4 & 2.3 & 1.7 & 0.8 & 0.9 \\
\hline foreign & 0.8 & 0.8 & 1.1 & 1.5 & 1.5 & 1.3 & 1.3 & 1.2 & 1.2 & 1.2 \\
\hline Capital expenditure (total) & 12.4 & 14.4 & 15.7 & 12.9 & 12.6 & 12.0 & 15.1 & 14.8 & 15.0 & 14.9 \\
\hline Domestic & 7.9 & 9.2 & 10.5 & 6.0 & 5.8 & 6.2 & 10.9 & 9.9 & 10.2 & 10.3 \\
\hline o/w from oil revenue & $\ldots$ & $\cdots$ & $\cdots$ & $\ldots$ & $\cdots$ & $\cdots$ & 5.7 & 6.1 & 2.6 & 2.6 \\
\hline Foreign & 4.5 & 5.2 & 5.2 & 6.9 & 6.8 & 5.8 & 4.2 & 4.9 & 4.8 & 4.6 \\
\hline Arrears clearance and VAT refunds & -0.5 & -0.7 & -1.0 & -2.7 & -2.7 & -0.9 & -0.9 & -0.7 & -0.6 & -0.5 \\
\hline Overall balance $^{3}$ & -7.5 & -9.2 & -14.5 & -9.4 & -9.4 & -6.0 & -4.5 & -2.8 & -2.1 & -2.1 \\
\hline Discrepancy & 0.1 & -0.3 & 0.8 & 0.0 & 0.0 & 0.0 & 0.0 & 0.0 & 0.0 & 0.0 \\
\hline Total financing & 7.7 & 8.9 & 15.3 & 9.4 & 9.4 & 6.0 & 4.5 & 2.8 & 2.1 & 2.1 \\
\hline Divestiture receipts (net) & 0.0 & 0.8 & 4.0 & 0.0 & 0.0 & 0.0 & 0.0 & 0.0 & 0.0 & 0.0 \\
\hline Foreign (net) & 1.9 & 6.1 & 1.0 & 2.3 & 4.0 & 4.7 & 3.8 & 3.2 & 2.5 & 1.8 \\
\hline Exceptional financing (debt relief, bilateral) & 0.8 & 0.7 & 0.4 & 0.6 & 0.6 & 0.6 & 0.5 & 0.4 & 0.3 & 0.0 \\
\hline Domestic (net) & 5.0 & 1.3 & 9.8 & 6.5 & 4.8 & 0.8 & 0.2 & -0.8 & -0.8 & 0.3 \\
\hline Banking system & 0.4 & -2.3 & 7.1 & 6.5 & 2.6 & -1.7 & 0.1 & -0.4 & -0.4 & 0.2 \\
\hline Non-bank & 4.5 & 3.6 & 2.7 & 3.0 & 2.2 & 2.5 & 0.1 & -0.4 & -0.4 & 0.2 \\
\hline \multicolumn{11}{|l|}{ Memorandum items: } \\
\hline Total poverty spending & 10.6 & 9.4 & 9.5 & 8.4 & 8.4 & 8.4 & 8.4 & 8.4 & 8.4 & 8.4 \\
\hline HIPC and MDRI spending & 2.6 & 2.8 & 1.6 & 1.1 & 1.0 & 1.3 & 1.3 & 1.2 & 1.1 & 1.0 \\
\hline Tax exemptions & $\ldots$ & 1.6 & 2.7 & 2.2 & 2.2 & 1.7 & 1.8 & $\ldots$ & $\ldots$ & $\cdots$ \\
\hline Primary balance & -4.2 & -6.1 & -10.7 & -5.6 & -5.1 & -2.2 & -0.9 & 0.1 & -0.1 & -0.1 \\
\hline Domestic primary balance ${ }^{4}$ & -5.1 & -7.0 & -10.1 & -4.8 & -4.3 & -1.4 & -0.5 & 1.0 & 0.9 & 0.7 \\
\hline Total government debt & 42.0 & 51.2 & 58.2 & 66.4 & 66.4 & 66.6 & 66.0 & 63.7 & 64.7 & 64.9 \\
\hline Domestic debt & 24.8 & 26.4 & 29.0 & 28.5 & 28.5 & 24.9 & 22.1 & 19.6 & 20.6 & 21.9 \\
\hline External debt & 17.2 & 24.8 & 29.2 & 37.9 & 37.9 & 41.7 & 43.9 & 44.1 & 44.0 & 43.0 \\
\hline Annual oil revenue & $\cdots$ & $\cdots$ & $\cdots$ & $\cdots$ & $\cdots$ & 0.0 & 6.6 & 7.3 & 6.9 & 6.1 \\
\hline Oil Fund & $\ldots$ & $\ldots$ & $\ldots$ & $\ldots$ & $\ldots$ & 0.0 & 0.0 & 0.0 & 3.9 & 6.8 \\
\hline Nominal nonoil GDP (millions of GHc) & 11,672 & 14,046 & 17,618 & 21,404 & 21,577 & 25,146 & 28,532 & 31,046 & 34,541 & 38,656 \\
\hline
\end{tabular}

Sources: Ghanaian authorities; and IMF staff estimates and projections.

$1 /$ Above-the-line data for domestic recurrent and capital expenditure are presented on a cash basis.

2/ For 2010 onwards, reflects the projected operating deficit of the electricity company, given the utility tariff structure in place as of June 2009 .

$3 /$ Includes cumulative fiscal measures.

4/ Defined as total revenue plus VAT refunds (negative), less noninterest recurrrent spending and domestic capital expenditure. 
Table 2B. Ghana: Summary of Central Government Budgetary Operations, 2006-14 1/

\begin{tabular}{|c|c|c|c|c|c|c|c|c|c|c|}
\hline & \multirow[t]{2}{*}{2006} & \multirow[t]{2}{*}{2007} & 2008 & \multicolumn{2}{|c|}{2009} & \multirow{2}{*}{$\begin{array}{l}2010 \\
\text { Proj. }\end{array}$} & \multirow{2}{*}{$\begin{array}{c}2011 \\
\text { Proj. }\end{array}$} & \multirow{2}{*}{$\begin{array}{c}2012 \\
\text { Proj. }\end{array}$} & \multirow{2}{*}{$\begin{array}{l}2013 \\
\text { Proj. }\end{array}$} & \multirow{2}{*}{$\begin{array}{c}2014 \\
\text { Proj. }\end{array}$} \\
\hline & & & Est. & Budget & Art. IV & & & & & \\
\hline Total revenue and grants & 3,192 & 4,052 & 4,839 & 6,385 & 6,470 & 7,359 & 9,792 & 10,893 & 11,935 & 13,070 \\
\hline Revenue & 2,557 & 3,195 & 4,019 & 5,083 & 5,167 & 6,103 & 8,689 & 9,666 & 10,609 & 11,593 \\
\hline Tax revenue & 2,326 & 2,824 & 3,505 & 4,394 & 4,472 & 5,279 & 6,908 & 7,615 & 8,370 & 9,169 \\
\hline o/w Oil sector & $\ldots$ & $\ldots$ & $\ldots$ & $\ldots$ & $\ldots$ & $\ldots$ & 1,036 & 1,226 & 1,261 & 1,214 \\
\hline Indirect taxes & 1,066 & 1,307 & 1,533 & 1,917 & 1,939 & 2,280 & 2,514 & 2,735 & 3,043 & 3,405 \\
\hline Trade taxes & 542 & 577 & 719 & 923 & 968 & 1,148 & 1,303 & 1,418 & 1,577 & 1,765 \\
\hline Nontax revenue & 231 & 370 & 513 & 689 & 696 & 823 & 1,781 & 2,051 & 2,239 & 2,424 \\
\hline o/w Oil sector & $\ldots$ & $\ldots$ & $\ldots$ & $\ldots$ & & $\ldots$ & 847 & 1,034 & 1,108 & 1,158 \\
\hline Grants & 635 & 857 & 821 & 1,302 & 1,302 & 1,256 & 1,103 & 1,228 & 1,326 & 1,477 \\
\hline Total expenditure & 4,009 & 5,245 & 7,229 & 7,827 & 7,927 & 9,523 & 11,709 & 12,499 & 13,488 & 14,878 \\
\hline Recurrent Non-interest expenditure & 2,167 & 2,788 & 3,789 & 4,250 & 4,269 & 5,567 & 6,380 & 6,986 & 7,619 & 8,317 \\
\hline Wages and salaries & 1,137 & 1,419 & 1,988 & 2,534 & 2,536 & 2,933 & 3,237 & 3,473 & 3,727 & 4,000 \\
\hline Goods and services & 428 & 565 & 648 & 415 & 415 & 484 & 571 & 667 & 767 & 889 \\
\hline Transfers & 298 & 580 & 618 & 902 & 919 & 1,059 & 1,201 & 1,307 & 1,383 & 1,535 \\
\hline Energy, utility and related subsidies & 215 & 32 & 260 & 54 & 54 & 795 & 1,035 & 1,171 & 1,335 & 1,436 \\
\hline Energy sector social safety net & 37 & 28 & 48 & 54 & 54 & 64 & 75 & 76 & 77 & 77 \\
\hline Utility company $\&$ refinery subsidies ${ }^{2}$ & 177 & 4 & 212 & 0 & 0 & 730 & 960 & 1,096 & 1,258 & 1,359 \\
\hline Miscellaneous payments/Reserve Fund & 90 & 192 & 275 & 345 & 345 & 297 & 337 & 366 & 408 & 456 \\
\hline Interest costs & 393 & 440 & 679 & 825 & 940 & 948 & 1,021 & 918 & 687 & 801 \\
\hline domestic & 303 & 322 & 482 & 508 & 623 & 615 & 644 & 530 & 276 & 330 \\
\hline foreign & 90 & 118 & 197 & 317 & 317 & 333 & 377 & 388 & 412 & 471 \\
\hline Capital expenditure (total) & 1,449 & 2,017 & 2,760 & 2,752 & 2,718 & 3,009 & 4,308 & 4,595 & 5,181 & 5,760 \\
\hline Domestic & 921 & 1,291 & 1,844 & 1,276 & 1,242 & 1,555 & 3,098 & 3,085 & 3,520 & 3,999 \\
\hline $\mathrm{o} / \mathrm{w}$ from oil revenue & & & & & & & 1,883 & 2,260 & 1,036 & 1,160 \\
\hline Foreign & 528 & 726 & 916 & 1,476 & 1,476 & 1,454 & 1,209 & 1,510 & 1,661 & 1,760 \\
\hline Arrears clearance and VAT refunds & -63 & -100 & -168 & -577 & -577 & -220 & -243 & -227 & -216 & -201 \\
\hline Overall balance ${ }^{3}$ & -881 & $-1,293$ & $-2,558$ & $-2,018$ & $-2,034$ & $-1,509$ & $-1,284$ & -880 & -709 & -823 \\
\hline Discrepancy & 17 & -46 & 133 & 0 & 0 & 0 & 0 & 0 & 0 & 0 \\
\hline Total financing & 898 & 1,247 & 2,690 & 2,018 & 2,034 & 1,509 & 1,284 & 880 & 709 & 823 \\
\hline Divestiture receipts (net) & 1 & 115 & 707 & 0 & 0 & 0 & 0 & 0 & 0 & 0 \\
\hline Foreign (net) & 225 & 861 & 171 & 491 & 866 & 1,175 & 1,080 & 1,003 & 877 & 690 \\
\hline Exceptional financing (debt relief, bilateral) & 93 & 92 & 78 & 135 & 135 & 138 & 145 & 115 & 115 & 0 \\
\hline Domestic (net) & 579 & 180 & 1,735 & 1,392 & 1,033 & 195 & 59 & -238 & -283 & 133 \\
\hline Banking system & 52 & -324 & 1,255 & 1,392 & 553 & -434 & 29 & -119 & -142 & 67 \\
\hline Non-bank & 535 & 504 & 479 & 0 & 480 & 629 & 29 & -119 & -142 & 67 \\
\hline Nominal nonoil GDP (millions of GHc) & 11,672 & 14,046 & 17,618 & 21,404 & 21,577 & 25,146 & 28,532 & 31,046 & 34,541 & 38,656 \\
\hline
\end{tabular}

1/ Above-the-line data for domestic recurrent and capital expenditure are presented on a cash basis.

2/ For 2010 onwards, reflects the projected operating deficit of the electricity company, given the utility tariff structure in place as of June 2009 .

3 / Including cumulative fiscal measures. 
Table 3. Ghana: Monetary Survey, 2006-14

\begin{tabular}{|c|c|c|c|c|c|c|c|c|c|}
\hline & $\begin{array}{l}2006 \\
\text { Dec. }\end{array}$ & $\begin{array}{l}2007 \\
\text { Dec. }\end{array}$ & $\begin{array}{r}2008 \\
\text { Dec }\end{array}$ & $\begin{array}{r}2009 \\
\text { Dec } \\
\text { Proj. }\end{array}$ & $\begin{array}{r}2010 \\
\text { Dec } \\
\text { Proj. }\end{array}$ & $\begin{array}{r}2011 \\
\text { Dec } \\
\text { Proj. }\end{array}$ & $\begin{array}{r}2012 \\
\text { Dec } \\
\text { Proj. }\end{array}$ & $\begin{array}{r}2013 \\
\text { Dec } \\
\text { Proj. }\end{array}$ & $\begin{array}{r}2014 \\
\text { Dec } \\
\text { Proj. }\end{array}$ \\
\hline \multicolumn{10}{|c|}{ (Annual percentage change) } \\
\hline Reserve money & 32.3 & 30.5 & 27.1 & 16.5 & 14.9 & 24.3 & 17.0 & 13.2 & 13.8 \\
\hline Broad money (M2+) & 38.8 & 35.9 & 40.2 & 21.7 & 16.9 & 27.9 & 23.0 & 18.9 & 17.8 \\
\hline Currency & 27.0 & 27.7 & 27.8 & 23.2 & 17.2 & 14.1 & 9.4 & 11.9 & 12.6 \\
\hline Deposits & 43.1 & 38.6 & 43.8 & 21.3 & 16.9 & 31.6 & 26.1 & 20.3 & 18.8 \\
\hline \multicolumn{10}{|c|}{ (Contribution to reserve money growth, in percent) } \\
\hline Monetary authorities & & & & & & & & & \\
\hline Net foreign assets of reserve bank & 58.4 & 42.9 & -30.9 & 10.0 & 20.9 & 60.0 & 54.8 & 45.2 & 39.9 \\
\hline Net domestic assets of reserve bank & -26.1 & -12.3 & 57.9 & 6.5 & -6.0 & -35.7 & -37.8 & -32.1 & -26.1 \\
\hline \multicolumn{10}{|l|}{ Of which } \\
\hline Credit to government (net) & -2.0 & -17.1 & 68.2 & 23.7 & 9.5 & -21.0 & -27.7 & -20.7 & -14.9 \\
\hline Credit to deposit money banks & 6.6 & 0.6 & 12.4 & 3.5 & -7.2 & -5.8 & -6.4 & -6.1 & -5.5 \\
\hline Credit to private sector & -0.3 & 0.2 & -0.2 & 0.0 & 0.0 & 0.0 & 0.0 & 0.0 & 0.0 \\
\hline Other items net (assets + ) & -30.5 & 4.0 & -22.4 & -20.7 & -8.4 & -9.0 & -3.8 & -5.2 & -5.7 \\
\hline Reserve money & 32.3 & 30.5 & 27.1 & 16.5 & 14.9 & 24.3 & 17.0 & 13.2 & 13.8 \\
\hline Currency outside banks & 21.4 & 21.1 & 20.7 & 17.4 & 13.6 & 11.4 & 7.0 & 8.3 & 8.6 \\
\hline Bank reserves & 9.9 & 10.5 & 4.3 & -0.8 & 1.3 & 12.8 & 10.0 & 4.9 & 5.2 \\
\hline \multirow[t]{2}{*}{ Nonbank deposits } & 1.0 & -1.0 & 2.1 & 0.0 & 0.0 & 0.0 & 0.0 & 0.0 & 0.0 \\
\hline & \multicolumn{9}{|c|}{ (Contribution to broad money growth, in percent) } \\
\hline \multicolumn{10}{|l|}{ Monetary survey } \\
\hline Net foreign assets & 20.4 & 8.1 & -6.0 & 3.8 & 5.9 & 13.2 & 12.0 & 9.8 & 8.3 \\
\hline Net domestic assets & 18.4 & 27.8 & 46.2 & 14.0 & 8.5 & 8.6 & 6.7 & 6.1 & 6.8 \\
\hline Claims on government (net) & 5.3 & -9.1 & 22.5 & 5.6 & -3.8 & 0.2 & -0.7 & -0.7 & 0.3 \\
\hline Claims on non-government $2 /$ & 24.0 & 38.5 & 31.9 & 14.0 & 14.6 & 10.5 & 8.2 & 8.0 & 7.8 \\
\hline PE's and cocoa & 3.8 & 9.3 & $\ldots$ & $\ldots$ & $\ldots$ & $\ldots$ & $\ldots$ & $\ldots$ & $\ldots$ \\
\hline Excl. PE's and cocoa & 20.2 & 29.2 & $\ldots$ & $\ldots$ & $\ldots$ & $\ldots$ & $\ldots$ & $\ldots$ & $\ldots$ \\
\hline Other items net (assets + ) & -10.9 & -1.6 & -8.2 & -5.6 & -2.3 & -2.1 & -0.9 & -1.2 & -1.2 \\
\hline Broad money (M2+) & 38.8 & 35.9 & 40.2 & 17.8 & 14.5 & 21.8 & 18.7 & 15.9 & 15.1 \\
\hline Currency & 7.1 & 6.7 & 6.3 & 3.9 & 3.1 & 2.3 & 1.4 & 1.7 & 1.7 \\
\hline Deposits & 31.7 & 29.3 & 33.9 & 13.9 & 11.4 & 19.5 & 17.2 & 14.2 & 13.5 \\
\hline \multicolumn{10}{|l|}{ Of which: } \\
\hline Foreign currency deposits & 8.0 & 2.1 & 14.3 & 8.6 & 4.2 & 7.0 & 7.8 & 7.3 & 6.4 \\
\hline \multirow[t]{2}{*}{ Broad money (M2) } & 30.9 & 33.8 & 25.9 & 9.2 & 10.3 & 14.8 & 10.8 & 8.6 & 8.7 \\
\hline & \multicolumn{9}{|c|}{ (Ratios, unless specified otherwise) } \\
\hline \multicolumn{10}{|l|}{ Memorandum items: } \\
\hline Currency-deposit ratio & 31.8 & 29.3 & 26.0 & 26.4 & 26.5 & 23.0 & 20.0 & 18.6 & 17.6 \\
\hline DMBs' reserves-deposit ratio (effective) & 9.4 & 9.9 & 8.1 & 6.5 & 5.9 & 7.7 & 8.6 & 8.3 & 8.2 \\
\hline Money multiplier (M2/reserve money) & 2.5 & 2.7 & 2.8 & 2.8 & 2.8 & 2.8 & 2.9 & 2.9 & 3.0 \\
\hline Velocity (GDP/M2) & 3.5 & 3.0 & 2.8 & 3.0 & 3.0 & 3.2 & 3.0 & 2.8 & 2.7 \\
\hline Real private sector credit growth (annual percentage change) & 28.7 & 41.6 & 25.5 & 9.0 & 15.0 & 10.0 & 10.0 & 10.0 & 10.0 \\
\hline
\end{tabular}

Sources: Ghanaian authorities; and IMF staff estimates and projections. 
Table 4. Ghana: Balance of Payments, 2006-14

\begin{tabular}{|c|c|c|c|c|c|c|c|c|c|c|}
\hline & 2005 & 2006 & $\begin{array}{r}2007 \\
\text { Est. }\end{array}$ & $\begin{array}{r}2008 \\
\text { Est. }\end{array}$ & $\begin{array}{r}2009 \\
\text { Proj. }\end{array}$ & $\begin{array}{c}2010 \\
\text { Proj. }\end{array}$ & $\begin{array}{c}2011 \\
\text { Proj. }\end{array}$ & $\begin{array}{c}2012 \\
\text { Proj. }\end{array}$ & $\begin{array}{c}2013 \\
\text { Proj. }\end{array}$ & $\begin{array}{r}2014 \\
\text { Proj. }\end{array}$ \\
\hline \multicolumn{11}{|c|}{ (Millions of US dollars) } \\
\hline Balance on Current Account (including grants) & -885 & $-1,265$ & $-1,805$ & $-3,110$ & $-1,869$ & $-2,362$ & $-1,732$ & $-1,616$ & $-1,986$ & $-1,359$ \\
\hline Trade balance & $-2,544$ & $-3,025$ & $-3,894$ & $-4,999$ & $-3,513$ & $-3,964$ & $-2,251$ & $-2,110$ & $-2,594$ & $-2,234$ \\
\hline Exports f.o.b. & 2,803 & 3,728 & 4,172 & 5,270 & 5,786 & 6,125 & 9,254 & 9,960 & 10,445 & 11,027 \\
\hline Cocoa & 908 & 1,187 & 1,133 & 1,487 & 1,976 & 1,978 & 1,916 & 1,827 & 1,859 & 1,976 \\
\hline Petroleum & 0 & 0 & 0 & 0 & 0 & 0 & 2,918 & 3,374 & 3,427 & 3,479 \\
\hline Other & 949 & 1,264 & 1,306 & 1,536 & 1,339 & 1,347 & 1,413 & 1,508 & 1,627 & 1,766 \\
\hline Imports, f.o.b. & $-5,347$ & $-6,754$ & $-8,066$ & $-10,268$ & $-9,299$ & $-10,089$ & $-11,505$ & $-12,069$ & $-13,040$ & $-13,261$ \\
\hline Non-oil & $-4,218$ & $-5,108$ & $-5,971$ & $-7,912$ & $-7,346$ & $-7,971$ & $-9,150$ & $-9,505$ & $-10,273$ & $-10,333$ \\
\hline Oil & $-1,129$ & $-1,646$ & $-2,095$ & $-2,357$ & $-1,953$ & $-2,119$ & $-2,355$ & $-2,565$ & $-2,766$ & $-2,929$ \\
\hline Balance on services and income & -354 & -277 & -305 & -758 & -442 & -480 & $-1,946$ & $-2,225$ & $-2,401$ & $-2,485$ \\
\hline Ut whıch: Interest payments & -160 & -110 & -87 & -130 & -162 & -158 & -162 & -161 & -165 & -184 \\
\hline Balance on transfers & 2,013 & 2,038 & 2,394 & 2,647 & 2,086 & 2,082 & 2,466 & 2,718 & 3,010 & 3,360 \\
\hline Official transfers (net) & 462 & 393 & 560 & 676 & 719 & 595 & 450 & 485 & 506 & 547 \\
\hline Capital and Financial Account & 1,002 & 1,933 & 2,365 & 2,443 & 1,215 & 2,437 & 2,594 & 2,623 & 2,889 & 2,211 \\
\hline Capital account (net) & 0 & 3,363 & 485 & 0 & 0 & 0 & 0 & 0 & 0 & 0 \\
\hline Of which: MDRI debt relief ${ }^{1}$ & 0 & 3,363 & 485 & 0 & 0 & 0 & 0 & 0 & 0 & 0 \\
\hline Financial Account (net) & 309 & $-2,472$ & 660 & 538 & 491 & 512 & 531 & 645 & 577 & 482 \\
\hline \multicolumn{11}{|l|}{ Official financing (medium and long-term loans) } \\
\hline Inflows & 500 & 501 & 1,279 & 730 & 686 & 693 & 755 & 775 & 718 & 718 \\
\hline Amortization & -191 & $-2,973$ & -619 & -192 & -195 & -181 & -224 & -131 & -141 & -236 \\
\hline Private capital & 559 & 1,019 & 1,061 & 2,307 & 1,114 & 1,950 & 2,063 & 1,928 & 2,263 & 1,679 \\
\hline Divestiture investments & & 0 & 115 & 900 & 0 & 0 & 0 & 0 & 0 & 0 \\
\hline Other FDI & & 636 & 855 & 1,212 & 1,084 & 1,900 & 1,983 & 1,828 & 2,163 & 1,575 \\
\hline Of which, oil-related FDI & & $\ldots$ & $\ldots$ & $\ldots$ & 398 & 1,095 & 1,095 & 663 & 806 & 0 \\
\hline Suppliers' credits and other & & 383 & 91 & 196 & 30 & 50 & 80 & 100 & 100 & 104 \\
\hline Short term capital & 134 & 23 & 159 & -402 & -250 & -25 & 0 & 50 & 50 & 50 \\
\hline Errors and omissions & 193 & -131 & -147 & -274 & -140 & 0 & 0 & 0 & 0 & 0 \\
\hline Financing & -309 & -537 & -413 & 941 & 654 & -75 & -862 & $-1,007$ & -904 & -852 \\
\hline Exceptional financing & $\ldots$ & $\ldots$ & $\ldots$ & $\ldots$ & 250 & 125 & 0 & & & \\
\hline Multilaterals & & $\ldots$ & $\ldots$ & $\ldots$ & 200 & 125 & 0 & & & \\
\hline IDA & & $\ldots$ & $\ldots$ & $\ldots$ & 200 & 125 & 0 & & & \\
\hline Others & & $\ldots$ & $\ldots$ & $\ldots$ & 0 & 0 & 0 & & & \\
\hline Official Bilaterals & & $\cdots$ & $\ldots$ & $\ldots$ & 50 & 0 & 0 & & & \\
\hline Paris Club & & $\ldots$ & $\ldots$ & $\ldots$ & 50 & 0 & 0 & & & \\
\hline Non-Paris Club & & $\ldots$ & $\ldots$ & $\ldots$ & 0 & 0 & 0 & & & \\
\hline Commercial & & $\ldots$ & $\ldots$ & $\ldots$ & 0 & 0 & 0 & & & \\
\hline Net foreign assets & -309 & -537 & -567 & 800 & 404 & -200 & -862 & $-1,007$ & -904 & -852 \\
\hline Net international reserves (-, incr.) & -309 & -537 & -567 & 800 & 404 & -200 & -862 & $-1,007$ & -904 & -852 \\
\hline Increase in gross reserves (-) & -309 & -537 & -413 & 941 & 86 & -400 & -950 & $-1,095$ & -904 & -811 \\
\hline Use of Fund credit & 0 & -265 & 0 & 0 & 200 & 200 & 88 & 88 & 0 & -41 \\
\hline Other NFA changes (increase -) & 0 & 0 & 0 & 0 & 118 & 0 & 0 & 0 & 0 & 0 \\
\hline Bilateral payment agreements & 0 & 0 & 0 & 0 & 0 & 0 & 0 & 0 & 0 & 0 \\
\hline \multicolumn{11}{|l|}{ Memorandum items: } \\
\hline Current account bal., incl. official transfers (\% of GDP) & -8.3 & -9.9 & -12.0 & -19.3 & -13.0 & -15.9 & -9.5 & -8.3 & -9.5 & -6.0 \\
\hline Current account bal., excl. official transfers (\% of GDP) & -12.6 & -13.0 & -15.8 & -23.5 & -18.0 & -19.9 & -11.9 & -10.8 & -11.9 & -8.5 \\
\hline Trade balance (percent of GDP) & -23.7 & -23.8 & -26.0 & -31.1 & -24.4 & -26.7 & -12.3 & -10.9 & -12.4 & -9.9 \\
\hline \multicolumn{11}{|l|}{ Gross international reserves ${ }^{2}$} \\
\hline In millions of US dollars & 1,895 & 2,270 & 2,837 & 2,036 & 1,950 & 2,350 & 3,300 & 4,395 & 5,299 & 6,110 \\
\hline In months of imports of goods and services & 2.7 & 2.7 & 2.7 & 2.2 & 1.9 & 2.0 & 2.7 & 3.4 & 4.0 & 4.3 \\
\hline External debt (percent of GDP) & 59.2 & 17.1 & 23.9 & 25.1 & 35.1 & 40.0 & 36.4 & 36.8 & 37.0 & 36.5 \\
\hline External debt service paid (in percent of exports of GNFS) & 14.7 & 11.8 & 3.7 & 4.6 & 4.8 & 4.2 & 3.4 & 2.4 & 2.4 & 3.2 \\
\hline Total donor support (percent of GDP) & 9.0 & 7.0 & 7.3 & 8.7 & 9.8 & 8.7 & 6.6 & 6.5 & 4.9 & 4.7 \\
\hline of which, official transfers (percent of GDP) & 4.3 & 3.1 & 3.7 & 4.2 & 5.0 & 4.0 & 2.5 & 2.5 & 2.4 & 2.4 \\
\hline Terms of trade $(1990=100)$ & 104 & 108 & 120 & 127 & 153 & 141 & 131 & 126 & 125 & 126 \\
\hline Change in the Terms of Trade & -6.9 & 4.4 & 11.5 & 5.3 & 20.3 & -7.3 & -7.4 & -3.8 & -1.2 & 1.2 \\
\hline GDP (US\$) & 10,726 & 12,729 & 14,984 & 16,085 & 14,385 & 14,870 & 18,321 & 19,423 & 20,872 & 22,505 \\
\hline
\end{tabular}

Sources: Bank of Ghana; and IMF staff estimates and projections.

${ }^{1}$ Including MDRI debt relief from the Fund and IDA in 2006, and the African Development Fund in 2007.

${ }^{2}$ Before possible new SDR allocation of US\$420 million in 2009. 
Table 5. Ghana: Financial Soundness Indicators, 2004-09

(Percent, end-of-period, unless otherwise specified)

\begin{tabular}{|c|c|c|c|c|c|c|}
\hline & 2004 & 2005 & 2006 & 2007 & 2008 & $\begin{array}{r}2009 \\
\text { March }\end{array}$ \\
\hline \multicolumn{7}{|l|}{ Capital adequacy: } \\
\hline Regulatory capital ratio & 13.9 & 16.2 & 15.8 & 14.8 & 13.8 & 14.6 \\
\hline Regulatory tier 1 capital ratio & 64.2 & 71.6 & 15.0 & 13.6 & 12.8 & 13.3 \\
\hline \multicolumn{7}{|l|}{ Asset quality: } \\
\hline Nonperforming loans to total gross loans & 16.3 & 13.0 & 7.9 & 6.4 & 7.7 & 9.6 \\
\hline Credit to total assets & 44.0 & 48.7 & 45.0 & 50.3 & 52.3 & 54.0 \\
\hline Loan loss provision to total gross loans & 13.8 & 8.5 & 7.4 & 5.5 & 6.3 & 7.6 \\
\hline \multicolumn{7}{|l|}{ Earnings and profitability: } \\
\hline Return on assets, before taxes (average) & 5.8 & 4.6 & 4.8 & 3.7 & 3.2 & 3.4 \\
\hline Return on equity, before taxes (average) & 33.7 & 23.6 & 39.6 & 35.8 & 23.7 & 21.6 \\
\hline Interest margin to gross income & 62.9 & 64.0 & 51.8 & 46.1 & 41.3 & 37.9 \\
\hline Interest spread $^{1}$ & 20.3 & 19.3 & 18.3 & 18.3 & 20.8 & 23.9 \\
\hline \multicolumn{7}{|l|}{ Liquidity: } \\
\hline Core liquid assets to total assets ratio & 25.4 & 20.7 & 23.5 & 23.4 & 25.2 & 22.6 \\
\hline Broad liquid assets to total assets ratio & 53.5 & 47.0 & 46.3 & 40.7 & 39.4 & 37.3 \\
\hline Core liquid assets to short-term liabilities ratio & 34.6 & 42.8 & 31.4 & 31.0 & 33.5 & 29.7 \\
\hline Broad liquid assets to short-term liabilities ratio & 72.8 & 97.4 & 61.9 & 54.1 & 52.4 & 49.1 \\
\hline \multicolumn{7}{|l|}{ Exposure to foreign exchange risk: } \\
\hline Share of foreign currency deposits in total deposits & 29.3 & 26.3 & 28.1 & 22.3 & 28.4 & 30.3 \\
\hline Share of foreign liabilities in total liabilities & 2.8 & 2.4 & 4.0 & 8.1 & 7.0 & 6.5 \\
\hline
\end{tabular}

Source: Bank of Ghana.

${ }^{1}$ Average lending rate minus average (saving and demand) deposit rate. 
Table 6. Ghana: External Financing Requirements and Sources, 2006-14 (millions of US dollars)

\begin{tabular}{|c|c|c|c|c|c|c|c|c|c|}
\hline & \multirow[b]{2}{*}{2006} & \multicolumn{2}{|c|}{ Est. } & \multicolumn{6}{|c|}{ IMF Staff Projections } \\
\hline & & 2007 & 2008 & 2009 & 2010 & 2011 & 2012 & 2013 & 2014 \\
\hline Total requirements & $-5,006$ & $-3,552$ & $-3,178$ & $-2,697$ & $-3,538$ & $-3,356$ & $-3,330$ & $-3,541$ & $-2,958$ \\
\hline Current account (excl. official transfers) & $-1,658$ & $-2,366$ & $-3,786$ & $-2,588$ & $-2,957$ & $-2,182$ & $-2,094$ & $-2,484$ & $-1,898$ \\
\hline Debt amortization & $-2,973$ & -619 & -192 & -195 & -181 & -224 & -131 & -141 & -236 \\
\hline Gross reserves accumulation & -375 & -567 & 800 & 86 & -400 & -950 & $-1,105$ & -915 & -824 \\
\hline Total sources & 5,006 & 3,552 & 3,178 & 2,697 & 3,538 & 3,356 & 3,330 & 3,541 & 2,958 \\
\hline IMF & -265 & 0 & 0 & 200 & 200 & 88 & 88 & 0 & -41 \\
\hline Other IFIs & 0 & 0 & 0 & 250 & 125 & 0 & 0 & 0 & 0 \\
\hline Capital flows & 5,270 & 3,552 & 3,178 & 2,247 & 3,213 & 3,268 & 3,242 & 3,541 & 2,999 \\
\hline Foreign direct investment (net) & 636 & 855 & 1,212 & 1,084 & 1,900 & 1,983 & 1,832 & 2,166 & 1,580 \\
\hline Disbursement from official creditors $1 /$ & 894 & 1,840 & 1,406 & 1,405 & 1,288 & 1,205 & 1,260 & 1,224 & 1,266 \\
\hline Other flows 2/ & 3,740 & 857 & 560 & -242 & 25 & 80 & 150 & 150 & 154 \\
\hline
\end{tabular}

Source: Ghanaian authorities and Fund staff calculations

$1 /$ includes loans and grants

$2 /$ includes all other net financial flows and errors and omissions 
Table 7. Ghana: Indicators of Capacity to Repay the Fund, 2009-2022 1/

\begin{tabular}{|c|c|c|c|c|c|c|c|c|c|c|c|c|c|c|}
\hline & 2009 & 2010 & 2011 & 2012 & 2013 & 2014 & 2015 & 2016 & 2017 & 2018 & 2019 & 2020 & 2021 & 2022 \\
\hline & \multicolumn{14}{|c|}{ Projections } \\
\hline \multicolumn{15}{|c|}{ Fund obligations based on existing credit (in millions of SDRs) } \\
\hline Principal & 0.0 & 0.0 & 10.5 & 21.1 & 21.1 & 21.1 & 21.1 & 10.6 & 0.0 & 0.0 & 0.0 & 0.0 & 0.0 & 0.0 \\
\hline Charges and interest & 0.7 & 0.8 & 0.8 & 0.7 & 0.6 & 0.5 & 0.4 & 0.3 & 0.3 & 0.3 & 0.3 & 0.3 & 0.3 & 0.3 \\
\hline \multicolumn{15}{|c|}{ Fund obligations based on existing and prospective credit (in millions of SDRs) } \\
\hline Principal & 0.0 & 0.0 & 10.5 & 21.1 & 21.1 & 21.1 & 54.9 & 67.6 & 71.7 & 77.5 & 77.5 & 43.7 & 20.5 & 5.8 \\
\hline Charges and interest & 0.8 & 1.9 & 2.3 & 2.6 & 2.6 & 2.5 & 2.3 & 1.9 & 1.6 & 1.2 & 0.8 & 0.5 & 0.4 & 0.3 \\
\hline \multicolumn{15}{|l|}{ Total obligations based on existing and prospective credit } \\
\hline In millions of SDRs & 0.8 & 1.9 & 12.9 & 23.7 & 23.7 & 23.5 & 57.2 & 69.5 & 73.3 & 78.7 & 78.3 & 44.2 & 20.8 & 6.1 \\
\hline In millions of US $\$$ & 1.3 & 2.8 & 19.5 & 35.9 & 36.0 & 35.9 & 87.1 & 105.9 & 111.6 & 119.9 & 119.3 & 67.3 & 31.7 & 9.4 \\
\hline In percent of gross international reserves & 0.1 & 0.1 & 0.6 & 0.8 & 0.7 & 0.6 & 1.2 & 1.4 & 1.6 & 1.7 & 1.6 & 0.9 & 0.5 & 0.1 \\
\hline In percent of exports of goods and services & 0.0 & 0.0 & 0.2 & 0.3 & 0.3 & 0.3 & 0.6 & 0.7 & 0.8 & 0.8 & 0.7 & 0.4 & 0.2 & 0.0 \\
\hline In percent of debt service $2 /$ & 0.4 & 0.8 & 4.9 & 11.4 & 10.9 & 8.1 & 14.6 & 13.9 & 6.5 & 10.0 & 8.2 & 3.9 & 1.6 & 0.4 \\
\hline In percent of GDP & 0.0 & 0.0 & 0.1 & 0.2 & 0.2 & 0.2 & 0.4 & 0.4 & 0.4 & 0.4 & 0.4 & 0.2 & 0.1 & 0.0 \\
\hline In percent of quota & 0.2 & 0.5 & 3.5 & 6.4 & 6.4 & 6.4 & 15.5 & 18.8 & 19.9 & 21.3 & 21.2 & 12.0 & 5.6 & 1.7 \\
\hline \multicolumn{15}{|l|}{ Outstanding Fund credit $2 /$} \\
\hline In millions of SDRs & 240.8 & 376.1 & 423.9 & 461.3 & 440.2 & 419.1 & 364.2 & 296.6 & 224.9 & 147.5 & 70.0 & 26.3 & 5.8 & 0.0 \\
\hline In millions of US\$ & 365.3 & 570.5 & 642.1 & 699.7 & 669.3 & 638.4 & 554.7 & 451.8 & 342.6 & 224.6 & 106.6 & 40.0 & 8.9 & 0.0 \\
\hline In percent of gross international reserves & 18.7 & 24.3 & 19.5 & 15.9 & 12.6 & 10.4 & 7.9 & 6.1 & 4.9 & 3.1 & 1.5 & 0.6 & 0.1 & 0.0 \\
\hline In percent of exports of goods and services & 4.8 & 7.0 & 5.6 & 5.7 & 5.3 & 4.8 & 3.9 & 3.1 & 2.3 & 1.4 & 0.7 & 0.2 & 0.0 & 0.0 \\
\hline In percent of debt service $2 /$ & 102.1 & 167.7 & 160.7 & 222.0 & 203.0 & 143.8 & 92.7 & 59.1 & 19.9 & 18.7 & 7.4 & 2.3 & 0.4 & 0.0 \\
\hline In percent of GDP & 2.5 & 3.8 & 3.5 & 3.6 & 3.2 & 2.8 & 2.3 & 1.8 & 1.3 & 0.8 & 0.3 & 0.1 & 0.0 & 0.0 \\
\hline In percent of quota & 65.2 & 101.9 & 114.9 & 125.0 & 119.3 & 113.6 & 98.7 & 80.4 & 61.0 & 40.0 & 19.0 & 7.1 & 1.6 & 0.0 \\
\hline Net use of Fund credit (in millions of SDRs) & 134.5 & 133.4 & 45.6 & 34.8 & -23.7 & -23.5 & -57.2 & -69.5 & -73.3 & -78.7 & -78.3 & -44.2 & -20.8 & -6.1 \\
\hline Disbursements & 135.3 & 135.3 & 58.4 & 58.4 & 0.0 & 0.0 & 0.0 & 0.0 & 0.0 & 0.0 & 0.0 & 0.0 & 0.0 & 0.0 \\
\hline Repayments & 0.8 & 1.9 & 12.9 & 23.7 & 23.7 & 23.5 & 57.2 & 69.5 & 73.3 & 78.7 & 78.3 & 44.2 & 20.8 & 6.1 \\
\hline \multicolumn{15}{|l|}{ Memorandum items: } \\
\hline Nominal GDP (in millions of US\$) & 14,385 & 14,870 & 18,321 & 19,423 & 20,872 & 22,505 & 24,167 & 25,441 & 27,001 & 28,845 & 30,898 & 33,166 & 35,802 & 38,857 \\
\hline Exports of goods and services (in millions of US\$) & 7,561 & 8,156 & 11,397 & 12,181 & 12,746 & 13,410 & 14,115 & 14,358 & 14,862 & 15,524 & 16,265 & 17,179 & 18,306 & 19,652 \\
\hline Gross international reserves (in millions of US $\$$ ) & 1,950 & 2,350 & 3,300 & 4,395 & 5,299 & 6,110 & 7,051 & 7,459 & 7,054 & 7,212 & 7,276 & 7,172 & 6,922 & 6,855 \\
\hline Debt service (in millions of US\$) $2 /$ & 357.6 & 340.2 & 399.6 & 315.2 & 329.7 & 444.0 & 598.6 & 763.9 & $1,722.9$ & $1,201.5$ & $1,446.4$ & $1,713.5$ & $2,030.6$ & $2,325.7$ \\
\hline Quota (millions of SDRs) & 369 & 369 & 369 & 369 & 369 & 369 & 369 & 369 & 369 & 369 & 369 & 369 & 369 & 369 \\
\hline
\end{tabular}

Tources: Fund staff estimates and projections.

1/ Includes prospective PRGF disbursements of SDR 387.45 million (105 percent of quota)

2/ Total debt service includes IMF repayments. 
Table 8. Ghana: Proposed Schedule of Disbursements Under the PRGF Arrangement, 2009-12 $1 /$

\begin{tabular}{|c|c|c|}
\hline Amount & Date Available & Conditions Necessary for Disbursement \\
\hline SDR 67.65 million & July 15,2009 & $\begin{array}{l}\text { Executive Board approval of the three-year PRGF } \\
\text { arrangement }\end{array}$ \\
\hline SDR 67.65 million & December 15, 2009 & $\begin{array}{l}\text { Observance of the performance criteria for } \\
\text { September } 30,2009 \text {, and completion of the first } \\
\text { review under the arrangement }\end{array}$ \\
\hline SDR 67.65 million & March 15, 2010 & $\begin{array}{l}\text { Observance of the performance criteria for } \\
\text { December } 31,2009 \text {, and completion of the second } \\
\text { review under the arrangement }\end{array}$ \\
\hline SDR 67.65 million & September 15, 2010 & $\begin{array}{l}\text { Observance of the performance criteria for June } 30 \text {, } \\
2010 \text {, and completion of the third review under the } \\
\text { arrangement }\end{array}$ \\
\hline SDR 29.22 million & March 15, 2011 & $\begin{array}{l}\text { Observance of the performance criteria for } \\
\text { December } 31,2010 \text {, and completion of the fourth } \\
\text { review under the arrangement }\end{array}$ \\
\hline SDR 29.21 million & September 15, 2011 & $\begin{array}{l}\text { Observance of the performance criteria for June } 30 \text {, } \\
\text { 2011, and completion of the fifth review under the } \\
\text { arrangement }\end{array}$ \\
\hline SDR 29.21 million & March 15, 2012 & $\begin{array}{l}\text { Observance of the performance criteria for } \\
\text { December } 31,2011 \text {, and completion of the sixth } \\
\text { review under the arrangement }\end{array}$ \\
\hline SDR 29.21 million & June 15,2012 & $\begin{array}{l}\text { Observance of the performance criteria for March } \\
31,2012 \text {, and completion of the seventh review } \\
\text { under the arrangement }\end{array}$ \\
\hline SDR 387.45 million & \multicolumn{2}{|c|}{ Total for the PRGF arrangement } \\
\hline
\end{tabular}

\footnotetext{
${ }^{1}$ In addition to the generally applicable conditions under the Poverty Reduction and Growth Facility arrangement.
} 
Table 9. Ghana: Selected Indicators on the Millennium Development Goals, 1990-2007

\begin{tabular}{|c|c|c|c|c|}
\hline & 1990 & 1995 & 2000 & 2007 \\
\hline \multicolumn{5}{|l|}{ Eradicate extreme poverty and hunger } \\
\hline Income share held by lowest $20 \%$ & 7.0 & .. & 5.6 & 5.2 \\
\hline Malnutrition prevalence, weight for age ( $\%$ of children under 5 ) & 24.1 & 25.1 & 20.3 & .. \\
\hline Poverty headcount ratio at $\$ 1.25$ a day (PPP) ( $\%$ of population) & 49 &.. & 39 & 30 \\
\hline Prevalence of undernourishment (\% of population) & 34 & 16 & .. & 9 \\
\hline \multicolumn{5}{|l|}{ Achieve universal primary education } \\
\hline Literacy rate, youth female (\% of females ages $15-24$ ) & .. & .. & 65 & 76 \\
\hline Persistence to last grade of primary, total (\% of cohort) & 78 &.. & 59 & .. \\
\hline Primary completion rate, total ( $\%$ of relevant age group) & 61 &.. & 62 & 71 \\
\hline Total enrollment, primary (\% net) & .. & .. & 61 & 72 \\
\hline \multicolumn{5}{|l|}{ Promote gender equality and empower women } \\
\hline Proportion of seats held by women in national parliaments (\%) & .. & .. & 9 & 11 \\
\hline Ratio of female to male enrollments in tertiary education & .. & .. & 34 & 54 \\
\hline Ratio of female to male primary enrollment & 84 & 90 & 93 & 99 \\
\hline \multicolumn{5}{|l|}{ Reduce child mortality } \\
\hline Immunization, measles ( $\%$ of children ages $12-23$ months) & 61 & 70 & 84 & 95 \\
\hline Mortality rate, infant (per 1,000 live births) & 76 & 71 & 71 & 73 \\
\hline Mortality rate, under-5 (per 1,000) & 120 & 111 & 112 & 115 \\
\hline \multicolumn{5}{|l|}{ Improve maternal health } \\
\hline Adolescent fertility rate (births per 1,000 women ages $15-19$ ) & .. & 86 & 77 & 55 \\
\hline Births attended by skilled health staff ( $\%$ of total) & 40 & 44 & 44 & 50 \\
\hline Maternal mortality ratio (modeled estimate, per 100,000 live births) & .. & .. & .. & 560 \\
\hline Pregnant women receiving prenatal care (\%) & 82 & 86 & 88 & 92 \\
\hline \multicolumn{5}{|l|}{ Combat HIVIAIDS, malaria, and other diseases } \\
\hline Children with fever receiving antimalarial drugs ( $\%$ of children under age 5 with fever) & .. & .. & 61 & 61 \\
\hline Incidence of tuberculosis (per 100,000 people) & 223 & 217 & 211 & 203 \\
\hline Prevalence of HIV, total (\% of population ages 15-49) & 0.1 & 1.8 & 2.4 & 1.9 \\
\hline \multicolumn{5}{|l|}{ Ensure environmental sustainability } \\
\hline CO2 emissions (metric tons per capita) & 0.2 & 0.3 & 0.3 & 0.3 \\
\hline Improved water source (\% of population with access) & 56 & 64 & 72 & 80 \\
\hline Nationally protected areas (\% of total land area) &.. & .. &.. & 15.9 \\
\hline \multicolumn{5}{|l|}{ Develop a global partnership for development } \\
\hline Aid per capita (current US\$) & 36 & 36 & 30 & 49 \\
\hline Debt service (PPG and IMF only, \% of exports, excluding workers' remittances) & 36 & 22 & 24 & 3 \\
\hline Internet users (per 100 people) & 0.0 & 0.0 & 0.1 & 3.8 \\
\hline Mobile cellular subscriptions (per 100 people) & 0 & 0 & 1 & 32 \\
\hline Telephone lines (per 100 people) & 0 & 0 & 1 & 2 \\
\hline \multicolumn{5}{|l|}{ Other } \\
\hline Fertility rate, total (births per woman) & 5.7 & 5.1 & 4.6 & 3.8 \\
\hline GNI per capita, Atlas method (current US\$) & 380 & 350 & 320 & 590 \\
\hline GNI, Atlas method (current US\$) (billions) & 5.8 & 6.3 & 6.5 & 13.8 \\
\hline Gross capital formation (\% of GDP) & 14.4 & 20.0 & 24.0 & 33.7 \\
\hline Life expectancy at birth, total (years) & 57 & 58 & 58 & 60 \\
\hline Literacy rate, adult total ( $\%$ of people ages 15 and above) &.. & .. & 58 & 65 \\
\hline Population, total (millions) & 15.6 & 17.9 & 20.1 & 23.5 \\
\hline Employment to population ratio, $15+$, total $(\%)$ & 69 & 67 & 67 & 65 \\
\hline Trade ( $\%$ of GDP) & 42.7 & 57.4 & 116.0 & 92.9 \\
\hline
\end{tabular}

Source: World Development Indicators database, 2009. 


\section{APPENDIX I-LETTER OF INTENT}

June 26, 2009

Mr. Dominique Strauss-Kahn

Managing Director

International Monetary Fund

Washington, DC 20431

Dear Mr. Strauss-Kahn:

After another peaceful, democratic and competitive election in Ghana in December 2008, a new government of the National Democratic Congress Party took over power in January 2009. The Government sees its political mandate as an opportunity to manage the economy to achieve prosperity for all Ghanaians.

While the country achieved significant gains in the macroeconomic and social areas through 2005 , the last three years were characterized by severe imbalances brought on by external shocks that were compounded by expansionary domestic policies.

The new government is committed to addressing the economic imbalances, re-stabilizing the economy, and placing it on a path of sustained high growth in order to accelerate the pace of progress toward the Millennium Development Goals and middle income status for Ghana.

The attached Memorandum of Economic and Financial Policies (MEFP) reviews recent economic developments and policies, and outlines adjustment and reform policies intended under the program. In support of the policies in the MEFP, the government requests that the Executive Board of the IMF approve a Poverty Reduction and Growth Facility (PRGF) arrangement with access in the amount of SDR 387.45 million (105 percent of quota).

The Government of Ghana will provide such information as the Fund may request in connection with progress in implementing its economic and financial policies. The government believes that the policies set out in the attached MEFP are adequate to achieve the objectives of its program, but it will take any further measures that may become appropriate for this purpose. The Government will consult with the Fund on the adoption of these measures, and in advance of revisions to the policies contained in the MEFP, in accordance with the Fund's policies on such consultations. 
Sincerely yours,

Dr. Kwabena Duffuor

Minister of Finance and

Dr. Paul A. Acquah

Economic Planning

Governor

Bank of Ghana 


\section{APPENDIX I-ATTACHMENT I \\ Memorandum of Economic And Financial Policies, 2009-12}

\section{Introduction}

1. The Government of Ghana has adopted a comprehensive program of macroeconomic stabilization and reform. This memorandum sets out Ghana's economic and financial policies for the period July 2009-June 2012, to be supported by the International Monetary Fund under a three-year PRGF arrangement.

2. Ghana successfully went through a keenly contested presidential and parliamentary elections in December 2008. A clear winner emerged after two rounds of balloting in the presidential elections with a slim majority. There was, subsequently, a smooth handover of power from the New Patriotic Party to the National Democratic Congress, the second such transfer since Ghana ushered in multi-party democratic governance in 1992. This outcome is a manifestation of the consolidation of the democratic gains, making Ghana a strong functioning democracy in Africa, and stands as the single most remarkable achievement in the country's governance history.

\section{Recent economic and social achievements}

3. Real GDP growth increased steadily from 3.7 percent in 2000 to 7.3 percent in 2008 . This growth was fostered by significant debt relief which provided the country with fiscal space to embark on critical infrastructure investments, particularly in the energy and road sectors, as well as targeted social spending, all under the Ghana Poverty Reduction Strategy (GPRS). The combination of higher output growth, declining inflation, and improved social spending under the GPRS framework contributed significantly to lower poverty levels. The national incidence of poverty declined from 39.5 percent in 1998/99 to 28.5 percent in 2005/06. At this rate, Ghana is poised to achieve the Millennium Development Goal (MDG) of halving extreme poverty ahead of 2015 .

4. In the education sector, gross enrolment ratios have increased. A major initiative for improved enrolment ratios was the abolition of mandatory school fees for basic education and the introduction of capitation grants in the 2005-06 academic year.

5. In the health sector, there have been progressive improvements in the delivery of a number of important outputs. Most notable are: increase in life expectancy from 55 years in 2003 to 57.9 in 2006; the introduction of a pre-paid National Health Insurance Scheme in 2004; and the introduction of free maternal care for expectant mothers. These, together, have put healthcare within the reach of the poor and vulnerable groups. 
6. Gender disparities are gradually declining in some areas of service provision, such as in primary education, where the country has almost achieved gender parity. Recent estimates suggest that gross enrolment ratios have been higher for girls than for boys putting Ghana on track to achieve MDG 3 (gender parity in primary enrolment).

7. The improved macroeconomic environment during the period paved the way for Ghana to make a debut on the international capital markets in October 2007, raising US\$750 million as additional capital targeted at infrastructural development for growth, especially in the key area of energy.

8. In the financial sector, important structural and institutional reforms have also been undertaken recently. In particular, a comprehensive legal and regulatory framework and strengthened risked-based prudential supervision policies have been put in place to further deepen the financial sector and safeguard the safety and soundness of the financial system.

\section{Macroeconomic stress during 2007-08}

9. In spite of the progress in the macroeconomic and structural areas, the economy has come under severe stress since 2006. The macroeconomic situation deteriorated sharply on the back of both domestic and external shocks. In 2006-07, Ghana suffered a severe energy crisis as a result of severe drought, leading to a significant shift from a predominant hydro to thermal power generation at a time of rising crude oil prices, with adverse impacts on the economy.

10. The global food and fuel price increases in 2007-08 adversely impacted most subSaharan African countries, including Ghana. In the context of these global shocks and the 2008 elections, public sector spending increased substantially, raising the fiscal deficit from 7.5 percent of GDP in 2006 to 14.5 percent of GDP in 2008. Contributing to the strong fiscal expansion were high energy-related subsidies, increased infrastructure investment, higher wages and salaries, and a rise in social mitigation expenditures to dampen the effects of the global price shocks.

11. The global financial crisis has contributed to further balance of payments pressures. While export proceeds have not, thus far, been significantly impacted, private remittances are slowing, there has been some outflow of portfolio investments, and the outlook for foreign direct investment is not encouraging. Official access to global market financing is now extremely limited. This has reinforced the urgency of macroeconomic adjustment and efforts to identify new external financing from development partners.

12. Strong public spending growth, combined with rapid credit expansion and rising oil import costs contributed to a widening of the external current account deficit from 9.9 percent of GDP in 2006 to 19.3 percent of GDP in 2008. In 2008, the overall balance of 
payments recorded a deficit of US\$941 million compared with a surplus of US\$413 million in 2007 (inclusive of the balances on sovereign bond proceeds). The 2008 deficit was mainly financed by a drawdown of reserves, leading to a decline in the stock of gross international reserves, by US $\$ 800$ million to US $\$ 2,036$ million at the end of 2008 , equivalent to 2.2 months of import cover.

13. Real GDP growth for 2008 remained strong at an estimated 7.3 percent, up from 5.7 percent in 2007. The pass through from higher global commodity price shocks, combined with fiscal expansion, resulted in a rise in headline inflation from 12.7 percent in December 2007 to 18.1 per cent by end-2008. The Bank of Ghana responded by increasing the prime rate by a cumulative 350 basis points in 2008 , ending the year at 17 percent.

14. Inflationary pressures have remained strong in the first four months of 2009, with 12-month inflation in the 20 percent range from January through April 2009. This reflects sharp increases in some components of the non-food category of the inflation basket, mainly as a result of sharp depreciation of the cedi against the major international currencies in the first quarter. The prime rate was further increased by another 150 basis points to 18.5 percent in February 2009, and the Bank of Ghana stands ready to tighten further as needed.

15. The Ghana cedi weakened against the US dollar and the Euro following a surge in demand for foreign exchange in 2008. In the interbank market, the Cedi depreciated by 38 percent against the US dollar. The larger than expected twin deficits of 2008, the drawdown in reserves, and the uncertainties in the domestic and global financial markets continued to weigh heavily on the Ghana cedi in the first quarter of 2009, with the cedi registering a further 16 percent against the dollar through end-May.

16. The banking system remains sound, well capitalized and fairly liquid. In 2008, the sector witnessed a reduction in concentration, intensified competition and expansion in branch networks.

\section{The Government's Economic Program}

\section{Medium-term objectives}

17. The Government is committed to pursuing measures that will ensure the attainment of macroeconomic stability. The strategy will be accomplished through fiscal discipline that hinges on prudent public expenditure management, enhanced domestic revenue mobilization, adherence to public procurement rules, restructuring of the utility companies to reduce the subsidy burden to the budget, public sector reforms, with particular emphasis on wage reforms, and encouraging the private sector to participate in the accelerated growth agenda through Public Private Partnerships (PPPs). 
18. To restore and consolidate fiscal and debt sustainability, the government intends to reduce the fiscal deficit (excluding divestiture receipts) from 14.9 percent of GDP in 2008 to 9.4 percent in $2009,6.0$ percent in 2010, and to 4.5 percent in 2011 . Further reduction is planned beyond 2011. Deficit reduction will be driven, to the extent possible, by cuts in low-priority public spending, with a view to reducing public expenditure in relation to GDP, and shifting the balance from recurrent spending to infrastructure investments. Strengthened revenue mobilization would aim to increase revenues in relation to GDP. Consistent with the fiscal deficit targets, domestic financing of the budget would be reduced to low levels over the medium term. This would free financial resources for private sector investments and job creation.

19. To help achieve the above fiscal goals, tax reforms and improved revenue administration will enhance the revenue mobilization effort. There will also be decisive measures to improve public expenditure management, enhance public financial management, restructure state-owned enterprises (SOEs), in particular the utility companies, and ensure the success of the public sector reforms.

20. In line with the above policies, the macroeconomic goals for 2011 include real nonoil GDP growth of a little over 6 per cent; gross international reserves close to three months of import cover; and stabilization of the total public debt relative to GDP, and with subsequent reduction to reduce risks of debt distress over the medium term.

21. Monetary policy will support the government's fiscal consolidation efforts over the medium term with a focus on stabilizing price and exchange rate expectations. Under the inflation targeting (IT) framework, monetary policy will aim at achieving a medium term inflation target of 7-9 percent within a forward looking time horizon of 18-24 months.

\section{Macroeconomic program for 2009}

22. The government's 2009 budget, approved in March 2009, was based on a growth projection of 5.9 percent. Although the outlook for the global economy has subsequently deteriorated, the government anticipates that Ghana will be somewhat insulated from the global recession on account of buoyant prices for its two main exports, cocoa and gold, and because of strong rain-based agricultural production. For fiscal prudence, the revenue projections have been updated, to reflect revised nominal GDP estimates. The GDP deflator figures, which are somewhat higher than earlier estimated, reflect inflation developments in the first quarter. This is broadly offset by lower growth forecasts of 4.5 percent prepared by Fund staff which recognizes downside risks to external demand and financing in the context of the global recession. Based on these calculations the earlier budget revenue projections have been largely retained. If growth and revenue collections over-perform, this may provide scope to reduce the deficit below the budget target. At the same time, if growth and revenue collections fall short, the government has contingency plans to maintain the deficit target 
through cuts in discretionary spending, including by delaying non-critical investment projects.

23. The 2009 budget included a combination of expenditure savings and revenue measures. On the expenditure side, cuts were made in spending on overseas travels, workshops, and conferences; by reducing the number of Ministries from 27 to 23; eliminating subsidies to the Volta River Authority (VRA) for oil imports; eliminating transfers to the Bulk Oil Storage and Transport Company (BOST); delaying the contribution to the West Africa Gas Pipeline Escrow Account until 2010; reducing non-developmental capital spending; and by reallocating HIPC relief funds across programs to reflect the most recent poverty-reduction priorities. On revenues, collections are being enhanced by increasing the airport tax, road tolls, fees and charges, and by more effective application of the communication service tax.

24. Data through the first quarter of 2009 suggests that revenue and non-interest expenditures are broadly on track. However, with treasury bill interest rates remaining high through May 2009, interest costs are projected to exceed earlier estimates for 2009 as a whole. To achieve the targeted fiscal deficit of 9.4 percent of GDP, additional fiscal savings totaling 1.0 percent of GDP (GHф 209 million) have been identified. Specifically:

a) The provision in the 2009 budget for wage structure reform (GH\& 50 million) has been eliminated, due to the postponement of reform to 2010 and later;

b) The agreed 2009 salary increase for medical workers was lower than budgeted, yielding savings of GHф 34 million;

c) The 2009 allocation for domestic investment spending has been reduced by GHc31 million, by delaying purchases of vehicles, office equipment, and other miscellaneous items. Social protection programs, particularly in health, education, and sanitation, will be preserved;

d) Tax exemptions will be reduced, yielding projected savings of GHф 40 million. Effective June 17, 2009, special permits and general concessions will no longer be available on a discretionary basis, and the Ghana Investment Promotion Center will no longer have discretionary power to grant exemptions. These exemptions would be available, in future, only with parliamentary approval, through the Ministry of Finance.

e) Customs and excise revenue collections are more buoyant than earlier projected, and the 2009 revenue projection has been increased by GH $\notin 43$ million. 
f) The government is tabling legislation to establish a National Stabilization Levy, comprising an additional 5 percent profit tax, effective through end-2010, applicable to companies in the following sectors: banking, insurance, other financial services, communications, mining, and brewing. The projected yield in 2009 is GHф11 million, with a full-year yield in 2010 of GHф 22 million or more.

25. The government is confident that these measures will have a combined yield of at least 1 percent of GDP. As noted above, the government has contingency plans to maintain the deficit target through further cuts in discretionary spending, including by delaying noncritical investment projects, in the event of a fiscal shortfall.

26. To further strengthen expenditure control, a temporary pause has also been imposed on the issuance of commencement certificate for new investment projects, with on-going projects subject to a review in order to prioritize them and better sequence their implementation. The government also intends to review the cash management by earmarked funds to identify potential unused resources, provided that this does not jeopardize povertyreducing expenditures.

27. Based on potential domestic financing for the budget of 4.8 percent of GDP in 2009, and updated projections for external disbursements, Ghana has a financing gap of $\$ 250$ million. This would be covered by a Development Policy Loan from the World Bank which would augment planned World Bank support by $\$ 200$ million in 2009, and by exceptional budget support from development partners. In the event of a shortfall in foreign program grant and loan financing, the government will offset this in part through spending cuts and in part through increased resort to domestic financing, up to a maximum of GHф 75 million (see details in the Technical Memorandum of Understanding).

28. Fiscal tightening in 2009 , combined with a tight monetary policy stance, is projected to ease pressure on the balance of payments. With exceptional budget support and projected Fund financing, gross international reserves are projected at slightly below two months of import cover of goods and services at end-2009. To further strengthen Ghana's international reserves position, the authorities do not intend to draw on the proposed new SDR allocation.

29. Ghana intends to maintain and strengthen its inflation targeting regime. The disinflation process will be supported by fiscal consolidation, including as greater stability returns to the foreign exchange market. For 2009, the Bank of Ghana will aim for a progressive convergence on the medium-term inflation target range, with inflation projected to decline to a $12-$ month rate of $14 \frac{1}{2}$ percent by end-2009. 


\section{The Structural Reform Agenda}

\section{Strengthening fiscal institutions}

30. Achieving success in the medium term will depend on strengthening fiscal institutions and enhancing revenue mobilization and a strong public expenditure management agenda. Key policy initiatives are summarized below.

\section{Tax Policy and Tax Administration}

31. Ghana in the 1990s reformed its revenue administration and tax policy with some success. It created new administrative structures, introduced the value-added tax, and made changes to the personal income tax schedule in order to increase the incentives to work and save. The corporate tax has been successively reduced from $35 \%$ to $25 \%$. A decade and a half later, the tax system is beginning to face new challenges emanating especially from ad hoc changes to parent acts and the adoption of ad hoc exemptions and discretionary waivers. Against this background, the government requested IMF technical assistance on tax policy and revenue administration which was provided in April-May 2009. Their recommendations will inform the new directions for Ghana's tax system.

32. Tax policy. The government is committed to streamline the tax legislation process so that all legislation affecting the tax system is reviewed and tabled by the Ministry of Finance and Economic Planning. As a first step, the government is committed to conduct reviews by September to inform the 2010 Budget in the following areas:

a) A review will be conducted of the nature and scope of tax exemptions and discretionary waivers by sectors, beneficiaries, and their rationale, including those related to any remaining firm-level customs tariff exemptions, with the objective of phasing out unneeded tax holidays and other business incentives, and curtailing the broad discretion given to other institutions;

b) the number of zero-rated items (excluding exports) will be reviewed with the goal of eliminating the zero-rating, or, if necessary, converting some to VAT exemptions.

33. Over the medium-term, to broaden the tax base and minimize distortions, reviews will also be conducted on:

c) the VAT regime, especially the threshold and refunds regime to strengthen the effectiveness of the VAT;

d) options to simplify the taxation of small businesses below the VAT threshold; 
e) the coverage of the VAT to the service sector; and

f) the taxation of all investment income and the final taxation of dividends.

34. The base of the income tax will also be broadened to cover the large potential taxpayers in the private and informal sectors who currently are not paying any income tax. The retention of internally generated funds by MDAs will also undergo serious review.

35. Revenue administration. Ghana's three tax revenue agencies operate independently, share no activities and have very little exchange of information at the operational level. This results in high compliance cost for tax payers, high administrative costs for government, and high opportunities for tax evasion. The government intends to consolidate and centralize management of the three agencies, with a specific objective to merge the VAT and income tax services into a single, integrated tax administration. A modernization strategy for these and other aspects of revenue administration is to be developed and approved by Cabinet before end-2009.

36. Efforts will also be directed towards closing the various leakages from the tax system. Leakages have been associated with customs valuation and invoicing, transit goods, free zone exemptions and bonded warehousing facility; VAT collection and payment to the VAT office; and management of the withholding tax by the Internal Revenue Service (IRS). Preventive services of the Customs, Excise and Preventive Service will be strengthened to reduce smuggling and the abuse of the transit goods arrangement.

37. Oil revenue management. Revenues from our oil production activities are expected to come on board by 2011. Like all oil-producing economies, we are faced with a number of challenges, including how much of the oil revenues should be saved, how to insulate fiscal policy from fluctuations in oil prices, and how to protect the economy from possible exchange rate appreciation (the so-called Dutch Disease). The goal is to ensure a cautious phasing of petroleum revenues into the economy. This will be guided by the country's absorptive capacity, by the balance between how much to spend to accelerate growth and reduce poverty, and by how much to save for the future.

38. The government intends to continue with the national dialogue on the use of oil revenues and with the ongoing work on the technical details of oil revenue management. Work on a petroleum regulatory bill and an oil revenue management bill are ongoing and will benefit from an IMF technical assistance mission scheduled for June 2009. Public consultations on the allocation of petroleum revenues and on the guidelines for the management of the funds will be held prior to legislation. In this area, the government intends to ensure that oil revenues are fully and transparently included within the budget. To further ensure the transparent treatment of oil and gas revenues, the government intends to 
extend Ghana's participation in the Extractive Industry Transparency Initiative (EITI) to cover this sector, as well as fishing.

39. Enhancing Fiscal Responsibility. Recent experience suggests that maintaining fiscal discipline remains a major challenge in fiscal management. As first step to instituting fiscal responsibility laws, the government intends, in the short-term, to lay out some fiscal rules that will strengthen fiscal discipline and help ensure desired fiscal policy outcomes. These rules will also provide a solid institutional foundation for managing the oil revenues that are projected to come on stream in 2011.

\section{Expenditure management initiatives}

40. Although public financial management reforms have advanced in recent years, continued expenditure slippages and budget over-runs expose weaknesses in the system. Budgetary processes have improved over the years, but current developments indicate the lack of adequate institutional and human capacity (a) to track expenditures at the budget implementation stage and (b) to effectively manage commitments from the Ministries, Departments and Agencies. The result is the inability to block unapproved spending at the commitment stage, with risk of an over-run in commitments and emergence of domestic arrears. The first step to addressing the problem is to strengthen Public Expenditure Monitoring Unit (PEMU) in its human resources and in its processes of monitoring and reporting of expenditure paths. Another important step is the establishment of a cash management framework that will provide an early warning system for management.

41. Cash Management. As part of the resolve to strengthen fiscal management, the government has already adopted a strengthened cash management system involving the Ministry of Finance and Economic Planning, the Bank of Ghana, and the Controller and Accountant General. The three institutions have also initiated a process of developing a Treasury Single Account (TSA) which brings all government accounts under a single composite account structure. The TSA will allow for better cash flow management and better planning of financing the public sector borrowing requirement. Revenue Management and Expenditure Management Committees have been established to support the cash management framework and the work of the EPCC.

42. Budget management systems. To further strengthen the government's capacity for budget monitoring and control, it intends to press forward with plans for comprehensive adoption of an integrated financial management information system (IFMIS). The existing system has underperformed in the past due to technical factors, and has been rolled out to only a limited number of ministries. Accordingly, this system will be reviewed with a view to either developing a plan to address its shortcomings in advance of comprehensive adoption or to lay the basis for moving to an alternative system. This review will be conducted by end-December 2009. 
43. Other expenditure reforms. The efficiency of public spending will be enhanced by

re-introducing public expenditure tracking surveys; improving evaluation of investments and projects and identifying programs that do not work, and for which less should be spent or stopped altogether; strengthening performance orientation in the public sector through effective implementation of performance management and development system (PMDS); and strengthening the legal framework for public/private partnerships (PPPs).

\section{Public Sector Reform and Payroll Management}

44. The heavy burden of the public sector wage bill remains a major concern. Between 2002 and 2008, wages absorbed an average of 44.5 percent of total tax revenue. This number is expected to rise to 49.5 percent in 2009 . The main objective of the reform is to strengthen the link between public sector pay and productivity; maintain the competitiveness of public sector incomes relative to the private sector; and determine the optimal number of employees needed to efficiently deliver public services. The government intends to adopt a comprehensive program of public sector reform, with the support of the World Bank.

45. Payroll management. Incomplete automation has allowed Ministries, Departments, and Agencies (MDAs) to significantly exceed approved hiring budgets. The government is committed to strengthening the existing database management system for personnel and payroll data of all public sector institutions. Sixty-five of the 114 agencies have migrated to the new payroll database as at end of April 2009, though this accounts for just 4 percent of the total staff strength of the subvented agencies. The government intends to migrate all subvented agencies by end-September 2009. A committee would be established to monitor progress of the migration exercise.

46. Hiring controls. To contain the wage bill, the government has adopted a selective hiring freeze under which new recruitment is principally in the education and health services. At the same time, the validation of personnel into the centralized personnel database has been strengthened to ensure that new recruits are consistent with budgetary provisions. This will be comprehensive across the public sector including all subvented agencies by end-September 2009.

47. Payroll audits. An annual audit of the personnel database will be conducted by a joint team of the Auditor General and the Internal Audit Agency. In addition, as an initial exercise, a headcount of all staff of the Ghana Education Service will be conducted as part of the efforts to ensure fiscal consolidation whilst improving the personnel database in the sector. The headcount exercise is expected to be completed by end-September 2009.

48. Subvented agency restructuring. Over the medium-term, a functional review of the public service will be undertaken to provide an objective basis for a plan and schedule for right-sizing. The restructuring of subvented agencies will be stepped up, and those that are no 
longer relevant to the government's objectives will be liquidated, while those that need to be partially or fully commercialized will be instructed to do so within an agreed time frame. Institutional responsibilities for this reform effort will be established by end-2009 with a view to making good subsequent progress in auditing and restructuring the subvented agencies.

49. Pay reform. The government will ensure that discussions on a simplified "single spine" public sector salary structure take account of affordability within the medium-term wage and salary budget. Consistent with the government's objective of ensuring that the outcome of public pay negotiations informs the annual budget, the government intends to put in place measures to complete such negotiations before the Budget is prepared and passed by Parliament. It is, thus, expected that public sector salary and salary-related negotiations would be completed by September before the budget is finalized and submitted to Parliament in November.

\section{Statutory Funds}

50. Another area of institutional policy reform has to do with the increasing rigidities in the budget and the limiting effects on policy maneuvering. Current rigidities arise partly from the existence of a number of statutory funds and committed expenditure items determined by contracts and agreements with development partners. Excluding wages and salaries, the total of these payments in the provisional budget outturn for 2008 was 48.5 per cent of total payments, and for the 2009 the estimate is about 60 per cent. Government plans to review the legal provisions and management of some of these statutory funds, with the view to introducing some flexibilities and efficiencies in the overall fiscal management.

\section{Energy sector reform}

51. Financial restructuring. The balance sheets of the majority of the state-owned enterprises are under serious financial distress. Government plans to move speedily to ensure that the Power Sector Financial Restructuring and Recovery Study commissioned in May 2008 is completed. This study will review of the financial status of all three power sector utilities and propose a financial restructuring and recovery program that will ensure their long-term viability. The study is expected to produce a comprehensive financial recovery plan to improve upon financial operating ratios, reduce short-term indebtedness and reduce arrears of each of the power utilities to suppliers and creditors. Additionally, the Plan will clearly mark out VRA's core and non-core assets, effect full transfer of national transmission grid assets from VRA to GRIDCO and ring-fence assets and liabilities associated with GoGsponsored gas-turbine power projects. 
52. Energy subsidies. To address the cost of energy subsidies, the government intends to (a) retain bi-weekly price adjustments for petroleum pricing, which are designed to ensure cost recovery for the oil refinery and bulk importers; (b) adjust electricity pricing by end-2009 to bring the average tariff to cost-recovery levels - that is, to a level that will allow the VRA to cover, at a minimum, its operating costs without resort to government subsidies, other than the lifeline subsidy for low-income consumers, which cost GHф 46 million in 2008 ( 0.3 percent of GDP); and (c) the electricity tariff structure will be subject to quarterly reviews starting in 2010 to ensure continuing cost recovery.

\section{Management of Domestic Arrears}

53. Provision has been made within the budget to settle in $2009 \mathrm{GH} \notin 534$ million of domestic arrears carried over from 2008. MDAs have been required to report any other potential domestic arrears from 2008 by end-September 2009, and a due diligence process has been launched to validate reported liabilities. If the arrears from 2008 exceed the amounts provided for in the budget, MDAs will be required to settle them, where possible, within their 2009 budget ceilings. Where this is not possible, remaining amounts will be addressed as a priority in the 2010 budget. At the same time, to avoid possible new arrears pressures, all MDAs have been instructed to take steps to avoid over-commitments.

\section{Debt Management}

54. To support the government's goals for debt and fiscal sustainability, the Government will adopt a comprehensive public debt management strategy, providing a clear framework for borrowing, establishing the principles that should guide the debt manager's decision regarding the currency composition, maturity, interest rates and other risks of the debt portfolio. Under this strategy the government intends to explore all avenues for concessional financing, and will seek to avoid nonconcessional borrowing in foreign currency wherever possible. In some cases, high-return infrastructure projects may require market-related financing (such as for energy, road, and rail projects, and public private partnerships), and may not be feasible without state guarantees. The government will evaluate these projects on a case-by-case basis, based on economic rate of return, impact on debt sustainability, and alternatives for achieving the same developmental goals. The government will provide to the Fund a semi-annual listing of projects being considered for market-related foreign financing, with a first report provided at the time of the first program review. On this basis, the government would request that the limits under the PRGF arrangement imposed on 
contracting or guaranteeing new external nonconcessional debt be set so as to accommodate such projects. ${ }^{1}$

\section{Poverty reduction strategy}

55. To mitigate the risks associated with slower growth for low income and vulnerable groups, the social protection and safety nets will be strengthened and expanded. Current protection for the most vulnerable include "lifeline" scheme for utility costs, the Livelihood Empowerment Against Poverty (LEAP) cash transfer program, school feeding program, and free maternal care for almost 500,000 women. Others include grants and subsidies for basic education, and national youth employment program. New initiatives include the provision of school uniforms to about 1.6 million pupils in public basic schools in deprived communities throughout the country. Free exercise books will also be provided for every pupil in all public basic schools. In addition, the current capitation grant will be increased by 50 percent. In the context of a move to full cost recovery utility pricing, a review will be conducted of the possible need to expand support provided to poor households.

56. The current Ghana Poverty Reduction Strategy (GPRS) covers the period through end-2009. The government will shortly develop a strategy to extend the GPRS to 2010 and beyond. This extended GPRS will underpin the government's program of adjustment and reform described in this memorandum.

\section{Monetary Policy, Exchange Rate Policy, and Financial Sector Issues}

57. One of the key aspects of BOG's IT framework is to build confidence and anchor expectations. The 2007-08 global financial crisis and its domestic pass-through effect seems to have dislodged inflation and exchange rate expectations temporarily but the Central Bank remains committed to the path of disinflation. In line with this, the BOG has recently increased the prime rate by 150 basis points, to 18.5 percent. It is projected that inflationary pressures will begin to ease by the second half of 2009, paving the way for reduction in the policy rate. Policies will aim at reducing inflation below 10 percent during 2010.

58. To strengthen the IT framework, the BOG will continue to build its forecasting and analytical capacity to support monetary policy implementation, drawing on the experience of other IT countries. The BOG will liaise closely with Fund staff in this area. In addition, the

\footnotetext{
${ }^{1}$ During the coming year, the government has identified a potential need for debt guarantees during 2009-10 of up to US\$300 million to the Ghana National Petroleum Company (GNPC) to facilitate its participation in the first phase of a program to exploit offshore gas deposits starting in 2011. The project would be commercially viable, and is anticipated to involve majority private sector equity participation. Debt service would be financed from gas sales to domestic or regional refineries, and risks to Ghana's debt sustainability outlook would be low
} 
communication strategy, which includes a press conference after each MPC, will be further strengthened. The Bank of Ghana intends to restructure its website by end-year to consolidate and give greater prominence to its inflation targeting policies and goals, and provide more user-friendly access to MPC press releases, inflation reports, and other IT materials. This transparency will help to reshape public expectations and in the process provide a forum for greater accountability by the Bank of Ghana.

59. The Bank of Ghana will continue to maintain a flexible exchange rate regime, with foreign exchange market interventions limited to smoothing short-term fluctuations.

\section{Financial supervision and regulation}

60. The Bank of Ghana launched a multi year program for risk-based supervision of banks in 2007 which is supported by the Canadian Office of the Superintendent of Financial Institutions (OSFI). It has set 2010 as the implementation year for BASEL II and in collaboration with the banking industry, agreed to adopt the Standardized Approach for credit risk and operational risk, and Standardized Measurement Method for market risk. An updated Financial Sector Strategic Plan (FINSSPII), a blueprint for financial sector development, will be completed during 2009, with implementation targeted to start in 2010.

61. The Bank of Ghana is alert to the risks that weak governance structures and inadequate risk monitoring systems can play in the banking system, including in state-owned financial institutions. The Bank of Ghana is addressing risks in this area in the multi-year banking supervision program. Banks have been required to appoint compliance officers; the committee overseeing the BASEL II process meets quarterly to discuss risk management policies; and the Bank of Ghana liaises more closely with external auditors in conducting bank supervision to supplement information available from their written reports. Vigilance has led to early Bank of Ghana intervention in some cases of problems inconsistent with prudential regulations.

62. To further extend the capacity of the banking system to play a key role in a rapidly growing economy, the Bank of Ghana has increased the capital requirements of banks. The Bank of Ghana has set the new minimum capital requirement at GH $\notin 60$ million to be met by end of 2009. Banks with majority domestic shareholders however, are required to increase their minimum capital to GH $\notin 25$ million by end 2010 , and then to $\mathrm{GH} \notin 60$ million by end 2012.

63. Major financial sector reforms initiated recently and in the early stages of implementation will be brought to full implementation during the program period. Notable among these are (i) National Pensions Act, 2008 which will establish a regulatory framework for a three-tier pension system; (ii) the Financial Services Bill, to provide the legal and regulatory framework for providing nonbank financial services to non-residents; (iii) the full 
blown establishment of the Financial Intelligence Centre under the Anti Money Laundering Act., 2008; (iv) the Borrowers and Lenders Act; and (v) the Home Mortgage Finance Act.

64. The reform of the domestic debt market is an important component of our efforts to deepen financial markets and to improve the efficiency of the government's debt operations. Among the measures to be implemented in the medium term are a rationalization of the primary dealer system, the annual publication of an issue calendar to guide portfolio managers to plan an orderly rebalancing of their portfolios, and the implementation of a comprehensive investor relations program targeted at both domestic and international participants in the government debt market.

65. Given the extensive changes in Ghana's financial sector since the last Bank-Fund Financial Sector Assessment Program (FSAP) update, the government would welcome a new FSAP update at the earliest possible opportunity.

\section{Risks and Contingencies}

66. Key economic and financial downside risks to the program include lower-thanexpected remittances and private capital inflows, a more rapid than expected increase in global oil prices, and a greater than anticipated economic slowdown in trading partner countries. If these risks materialize, the government stands ready to adjust its policies, in close consultation with IMF staff, to ensure the achievement of a sustainable external position by the end of the program period.

\section{Data Provision and Program Monitoring}

\section{Statistics}

67. The following statistical data will be made available to the public as part of information dissemination strategy of Government and to allow for transparency in government operations:

- National accounts. With technical assistance from the IMF, the National Accounts numbers have been revised and rebased to 2004. The Ghana Statistical Service plans to publish the new numbers by end December 2009. The GSS also plans to produce quarterly national accounts after the publication of the new national accounts series.

- Inflation data. Inflation (CPI) data will be announced by the $10^{\text {th }}$ of the month following the one that the outturn relates to. The Prime Building Cost Index (PBCI) will be produced and disseminated quarterly. The Producer Price Index (PPI) will be produced and disseminated with a month's lag. 
- A population and housing census will be undertaken in 2010. Preparatory work has started, and the census report is expected to be produced and disseminated starting May 2011; and

- Public finance data. The Controller and Accountant General will release the monthly statement of the Final Accounts of government with a maximum of six weeks lag. The Ministry of Finance will publish monthly fiscal outturns on its website with a maximum lag of eight weeks, starting with the January to March 2009 accounts and historic monthly data for 2008, which will be published by end-June 2009. Annual data on the breakdown of public sector staff strength by MDA in 2008, together with corresponding data on the aggregate wage bill by MDA will be published on the Ministry's website by end-September 2009, with estimates for 2009 and subsequent years published within three months of end-year.

- Nonconcessional financing. The Ministry of Finance will provide a listing and status report for projects being considered for nonconcessional financing for end-June and end-December of each year (see para. 52).

\section{Program monitoring}

68. To ensure coordinated implementation of the government's program, the Economic Policy Coordinating Committee (EPCC), with participants from the Ministry of Finance, Bank of Ghana, and other affected ministries, departments, and agencies (MDAs) will meet on a regular basis to track progress under the various reform agendas. The EPCC, together with the Ministry of Finance's Public Expenditure Monitoring Unit, will have particular responsibility for oversight of public spending to ensure compliance with budget limits.

69. Furthermore, with the view to enhancing data dissemination and transparency, the Ministry of Finance will post a broad range of macroeconomic data on a timely manner on its website on a continuous basis, including (a) quarterly fiscal estimates, with a lag of no more than 8 weeks, starting with the 2009 first quarter accounts and comparable quarterly data for 2008; (b) the Controller and Accountant General's Department's (CAGD) monthly statement of the Final Accounts of the government with a maximum lag of 6 weeks; (c) annual data on the staff strength of public sector workers and aggregate wage bill, broken down by MDAs.

70. Quantitative performance criteria and structural benchmarks for 2009-2010 are set out in Tables 1 and 2. Progress on structural reforms in the key areas previously mentioned, namely tax policy and revenue administration, public expenditure management, public sector reform, energy sector reform, the inflation targeting framework, and financial supervision and regulation, will be monitored in the context of reviews. To facilitate such monitoring, structural benchmarks for 2009-10 are set out in Table 2. In addition, given the need for close monitoring in the context of an uncertain global environment, the program includes quarterly 
reviews for 2009 followed by semi-annual reviews starting in 2010. The first review of the program is expected to be completed by end-December 2009, with end-September 2009 as the test date for the quantitative performance criteria; the second and third reviews under the program are expected to be completed by end-April and end-October 2010, with endDecember 2009 and end-June 2010 as the test dates. Detailed definitions and reporting requirements for all performance criteria are contained in the Technical Memorandum of Understanding (TMU) attached to this letter.

71. We believe that the policies specified in the MEFP provide a basis for sustaining growth, reducing inflation, and alleviating poverty — but we stand ready to take additional measures if required. The government will provide the Fund with the information needed to assess progress in implementing our program as specified in the TMU, and will consult with the Fund staff on any measures that may be appropriate at the initiative of the Government or whenever the Fund requests a consultation. The Government intends to make this letter and the TMU available to the public. In this context, it authorizes the IMF to arrange for them to be posted on the Fund's website, subsequent to Executive Board approval.

72. Accordingly, we are requesting Board approval of the policies set forth in the MEFP, and disbursement of the first loan installment, totaling SDR 387.45 million. 


\begin{tabular}{|c|c|c|c|c|c|c|c|}
\hline & \multirow{2}{*}{$\begin{array}{l}2008 \\
\text { Dec. } \\
\text { Act. }\end{array}$} & \multicolumn{3}{|c|}{2009} & \multirow{2}{*}{$\begin{array}{r}\text { Dec } \\
\text { Prog. }{ }^{2}\end{array}$} & \multicolumn{2}{|c|}{2010} \\
\hline & & Mar. & $\begin{array}{ll}\text { Jun. } \\
\text { Proi. }\end{array}$ & $\begin{aligned} & \text { Sep. } \text {. } \\
& \text { Proq }^{2}\end{aligned}$ & & $\begin{array}{l}\text { Mar. } \\
\text { Proq }^{3}\end{array}$ & Jun. \\
\hline \multicolumn{8}{|l|}{ Quantitative Performance Criteria } \\
\hline Overall fiscal deficit of the government (ceiling; in millions of cedis) ${ }^{4}$ & 2,558 & 344 & 788 & 1470 & 2034 & 192 & 742 \\
\hline Net international reserves of the Bank of Ghana (floor; millions of U.S. dollars) ${ }^{5}$ & 1,301 & -404 & -637 & -728 & -404 & -81 & -37 \\
\hline \multicolumn{8}{|l|}{ Continuous Performance Criteria } \\
\hline Non-accumulation of external arrears (ceiling; millions of U.S. dollars) & 0 & 0 & 0 & 0 & 0 & 0 & 0 \\
\hline Contracting or guaranteeing of new external nonconcessional external debt (ceiling; millions of U.S. dollars) & $\ldots$ & $\ldots$ & $\ldots$ & 300 & 300 & 300 & 300 \\
\hline Oil and gas sector projects ${ }^{7}$ & $\ldots$ & $\ldots$ & $\ldots$ & 300 & 300 & 300 & 300 \\
\hline Other sectors & $\ldots$ & $\ldots$ & & 0 & 0 & 0 & 0 \\
\hline \multicolumn{8}{|l|}{ Inflation Consultation } \\
\hline \multicolumn{8}{|l|}{ Twelve-month consumer price inflation (percent) ${ }^{8}$} \\
\hline Outer band (upper limit) & $\ldots$ & $\ldots$ & $\ldots$ & 19.5 & 17.6 & 15.2 & 12.7 \\
\hline Inner band (upper limit) & & & & 18.5 & 16.6 & 14.2 & 11.7 \\
\hline Central target rate of inflation & 18.1 & 20.5 & 19.7 & 16.5 & 14.6 & 12.2 & 9.7 \\
\hline Inner band (lower limit) & $\ldots$ & $\ldots$ & $\ldots$ & 14.5 & 12.6 & 10.2 & 7.7 \\
\hline Outer band (lower limit) & $\ldots$ & $\ldots$ & $\ldots$ & 13.5 & 11.6 & 9.2 & 6.7 \\
\hline \multicolumn{8}{|l|}{ Indicative Target } \\
\hline Net domestic financing of the government (ceiling, in millions of cedis) ${ }^{9}$ & 479 & 243 & 591 & 828 & 1,033 & 47 & 92 \\
\hline Net domestic assets of the Bank of Ghana (ceiling; millions of cedis) ${ }^{10}$ & 299 & 41 & 382 & 662 & 146 & -103 & -128 \\
\hline
\end{tabular}

${ }^{1}$ All items as defined in the attached Technical Memorandum of Understanding (TMU). Performance criterion.

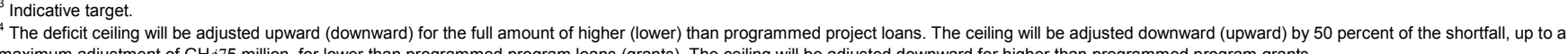

${ }^{5}$ Net international reserves at end-December 2008 was US $\$ 1,300.6$ million. The NIR floor will be adjusted upward by any new SDR allocation. The floor will be adjusted upward for higher than

programmed program loans and grants.

US $\$ 300$ million, cumulative from July 1,2009 .

${ }^{7}$ The projects would be conducted by the GNPC.

${ }^{8}$ Consultation arrangements triggered should inflation breach the inner or outer bands are described in the TMU.

${ }^{9}$ Indicative target. The ceiling will be adjusted upward by 50 percent of the shortfall, up to a maximum adjustment of $\mathrm{GH} \notin 75$ million, for a shorfall in program loans and grants. The ceiling will be adjusted

downward for higher than programmed program grants and loans.

${ }^{10}$ At program exchange rate (see TMU, paragraph 2). Net domestic assets at end-December 2008 was GH\&298.5 million. The NDA ceiling will be adjusted downward by any new SDR allocation. 
Table 2. Ghana: Structural Conditionality under PRGF Arrangement, 2009-2010 1/

Measures

\section{PRIOR ACTIONS}

- Adoption of budget to limit fiscal deficit to 9.4 percent of GDP in 2009.

- Adoption of measures yield at least 1.0 percent of GDP to offset projected expenditure over-runs in the 2009 budget.

- Selective public sector hiring freeze, with exemptions mainly limited to health and education trainees.

- Reinstatement of automatic bi-weekly price adjustments for petroleum products.

\section{Structural BenChMarks}

Tax policy and revenue administration

- Complete comprehensive reviews of zero-rated VAT items and the nature and scope of tax exemptions and discretionary waivers, as defined in paragraph 32 .

- Cabinet approval of a modernization strategy for revenue administration, as defined in paragraph 35 .

Public expenditure management

- Review of the effectiveness of the existing budget information management system, and decision on whether it should be modernized or replaced.

Public sector reform and payroll management

- Steps to strengthen oversight and control of public service recruitment and staffing, as defined in paragraphs 45-47.

- Establish institutional responsibility for the restructuring, commercialization, or liquidation of subvented agencies.

Monetary policy

- Adoption and launch of program to strengthen communication of framework for inflation targeting and disinflation over program period.
Timing

Macroeconomic rationale (MEFP para)

Implemented.

A first step toward fiscal and debt sustainability ( $\boldsymbol{\$} 18)$.

Implemented.

To preserve macroeconomic stability and avoid crowding out private sector credit ( $\mid 24)$.

Implemented. To strengthen control of the high and growing public payroll ( $(46)$.

Implemented. To eliminate energy subsidies ( $(\llbracket 52)$.

End-Sep. 2009 Strengthen revenue mobilization as part of the fiscal consolidation strategy $(\Phi 32)$.

End-Dec. 2009 Strengthen revenue mobilization as part of the fiscal consolidation strategy ( $₫ 35)$.

End-Dec. 2009 Strengthen monitoring and control of budget execution ( $(\llbracket 42)$.

End-Sep. 2009 To strengthen oversight and control of the high and growing public payroll ( $\mid 45-47)$.

End-Dec. 2009 To promote fiscal savings by rationalizing subvented agency numbers ( $(48)$.

End-Dec. 2009 To support the disinflation program ( $₫ 58)$.

1/ Structural conditionality for the third and fourth reviews, to cover the period through end-June and endDecember 2010 will be determined in the first and second reviews. 


\section{APPENDIX I-ATTACHMENT II}

\section{TeChNical Memorandum of Understanding}

\section{Arrangement Under the Poverty Reduction and Growth Facility July 2009-June 2012}

1. This technical memorandum of understanding (TMU) defines the variables subject to quantitative targets (performance criteria and indicative targets), as specified in the Letter of Intent (LOI). It also describes the methods to be used to assess the program performance and the information requirements to ensure adequate monitoring of the targets. The authorities will consult with the Fund before modifying measures contained in this letter, or adopting new measures that would deviate from the goals of the program, and provide the Fund with the necessary information for program monitoring.

2. Program exchange rate: The exchange rates for the purpose of the program of the Ghanaian cedi (GHф) to the U.S. dollar will be GH $\varnothing 1.45$ per US\$ 1 , which is calculated as the average of buying and selling exchange rates reported by banks to the Bank of Ghana as of June 19,2009. The exchange rates to other currencies will be calculated as the average of buying and selling exchange rates against the U.S. dollar as of June 19, 2009.

\section{Quantitative Program Indicators}

3. For program monitoring purposes, the performance criteria are set for end-September 2009, end-December 2009, and end-June 2010, while indicative target is set for end-March 2010. The performance criteria under the arrangement are: (a) a ceiling on the overall fiscal deficit (including grants) of the government; (b) a floor on the net international reserves of the Bank of Ghana; (c) a continuous zero ceiling for the accumulation of new external arrears; and (d) a ceiling on the contracting or guaranteeing of new external nonconcessional debt. Indicative targets are established as: (a) a ceiling on the net domestic financing of the government; and (b) as a ceiling on the net domestic assets of the Bank of Ghana. A target is set for the twelve-month rate of consumer price inflation, with triggers discussions or consultations with the Fund if inflation moves outside specified inner and outer bands. Performance criteria, indicative targets, and adjusters are calculated as cumulative flows from the beginning of the calendar year. 


\section{A. Government}

4. Definition: The government is defined as comprising the central government, all special funds (the Education Trust Fund, the Road Fund, the District Assembly Common Fund, and the National Health Insurance Fund), and all subvented and other government agencies that are classified as government in the Bank of Ghana (BOG) Statement of Accounts (SOA). The Social Security and National Insurance Trust (SSNIT) and public enterprises, including Cocobod, are excluded from the definition of government.

5. The fiscal deficit is defined as the difference between total expenditures and the sum of total revenues and total grants.

6. Revenues comprise all tax and nontax revenues of the government (both in cedi and in foreign currencies), excluding foreign grants and divestiture receipts. Revenue will be measured on a cash basis as gross inflows to the government's uncommitted treasury collections accounts (as reported by the BOG).

7. Expenditures comprise all spending from uncommitted accounts for Items 1-4, as captured by the accounts of the Controller and Accountant General's Department (CAGD), transfers, payments to statutory funds, HIPC-financed expenditure, and VAT refunds. Reporting will be based on the current National Expenditure Tracking System (NETS) accounting system and its associated 15-digit chart of accounts and will be fully reconciled with BOG bank statements on spending (outflows) from the ministries, departments, and agencies' (MDA's) operational accounts (plus any residual use of existing treasury drawing/overdraft accounts). Expenditure will also be verified by comparing it with accounts produced by the Budget and Public Expenditure Management System (BPEMS) accounting system, until such time as it becomes fully operational.

8. The government will continue to report poverty-related expenditures, including the use of funds from the enhanced Heavily Indebted Poor Countries (HIPC) Initiative. Budgeted poverty spending for these categories will be taken from each year's final appropriations bill and will include spending financed by the government or donors or from internally generated funds. Actual poverty-related spending will be identified using the last 3 digits of the 15-digit chart of accounts of the CAGD's current NETS and the subcomponent that is financed through HIPC Initiative debt relief. This data will be supplemented with that proportion of transfers to the District Assembly Common Fund, the Ghana Educational Trust Fund, and the Road Fund, which are deemed by those entities to be poverty-related. Accordingly, actual poverty spending will exclude some donor-supported expenditure not currently captured by the CAGD. 
9. Net domestic financing of government is defined as the change in net credit to government by the banking system (i.e., the Bank of Ghana plus deposit money banks) plus the net change in holdings of treasury bills and other government securities by the nonbank sector, excluding divestiture receipts. Such credit will also exclude treasury bills issued for open market operation purposes from January 1, 2003 onward (the holdings of which are excluded from the BOG Treasury Department's Debt Registry of central government securities, and the proceeds of which are sterilized in deposits held as other BOG liabilities, as defined in the monetary template provided to the IMF on December 3, 2003).

10. Outstanding net credit to the government by the Bank of Ghana comprises the sum of claims on government (SOA codes 0401 and 050101-4), including overdrafts of the government with the BOG, less government deposits (1101 including the main HIPC Initiative receiving account, and 1202) as defined in the monetary template.

11. Outstanding net credit by deposit money banks comprises deposit money bank (DMB) holdings of government securities at cost of purchase value, as reported by the BOG Treasury Department's Debt Registry, plus overdrafts less government deposits (the BOG's definition of government will be used for program purposes) as reported by DMBs in the revised BSD2 report forms (and defined in the Monetary Template), plus deferred accrued interest on their holdings of inflation-indexed bonds.

12. Nonbank financing is the difference between total net cash receipts to the treasury main cash account (issues/redemptions account when it becomes operational) from the sale/repurchase of government securities, less the corresponding net cash value received from the BOG and DMBs as indicated on the Debt Registry by holder at discount value, plus deferred accrued interest on their holdings of inflation-indexed bonds. For each test date, any adjustment by the BOG to the data reported by individual DMBs, on account of their misclassification of government or for other reasons, will be reported to the Fund.

\section{B. Consultation Mechanism on Inflation}

\section{The quarterly consultation band for the twelve-month rate of consumer price} inflation (as measured by the headline consumer price index (CPI) published by the Ghana Statistical Services) are specified in the text table: Inflation Targets. Projected CPI for endSeptember 2009, end-December 2009, and end-June 2010 will be subject to the consultation mechanism, while those for end-June 2009 and end-March 2010 are indicative targets. 
Text Table: Inflation Targets

\begin{tabular}{|l|c|c|c|c|c|}
\hline & Jun. 2009 & Sep. 2009 & Dec. 2009 & Mar. 2010 & Jun. 2010 \\
\hline Outer band (upper limit) & $\ldots$ & 19.5 & 17.6 & 15.2 & 12.7 \\
\hline Inner band (upper limit) & $\ldots$ & 18.5 & 16.6 & 14.2 & 11.7 \\
\hline Central inflation target & 19.7 & 16.5 & 14.6 & 12.2 & 9.7 \\
\hline Inner band (lower limit) & $\ldots$ & 14.5 & 12.6 & 10.2 & 7.7 \\
\hline Outer band (lower limit) & $\ldots$ & 13.5 & 11.6 & 9.2 & 6.7 \\
\hline
\end{tabular}

14. When the observed year-on-year CPI inflation rate falls outside a specific band, this would trigger consultation with the Fund.

- The authorities will complete consultation with the Executive Board of the Fund on the proposed policy response before requesting further purchases under the program when the observed year-on-year CPI inflation rate moves outside the outer band as specified for the end of each quarter in the above table. The authorities will not be able to request any further disbursements under the PRGF arrangement if inflation moves outside of the outer band until the consultation with the Executive Board has taken place.

- The authorities will conduct discussions with the Fund staff when the observed year-on-year CPI inflation rate falls outside the inner band as specified for the end of each quarter in the above table.

15. In line with our accountability principles, we are committed to report to the public the reasons for any breach of the outer bands, and our policy response.

\section{Bank of Ghana}

16. Net foreign assets are defined in the monetary survey as short- and long-term foreign assets minus liabilities of the BOG that are contracted with nonresidents. Short-term foreign assets include: monetary gold (valued at the spot market rate for gold, US\$/fine ounce, London), holdings of SDRs, reserve position and HIPC Initiative trust investment in the IMF, the HIPC Initiative Umbrella SDR account (all as reported by the IMF), foreign notes and travelers checks, foreign securities, positive balances with correspondent banks, and other positive short-term or time deposits. Short-term foreign liabilities include foreign currency liabilities contracted by the BOG at original maturities of one year or less (including 
overdrafts), outstanding liabilities to the IMF, and deposits of international institutions at the BOG. Long-term foreign assets and liabilities are comprised of: other foreign assets (BOG statement of accounts code 303), investments abroad (a subset of 60201), other long-term liabilities to nonresidents (a subset of 1103), and bilateral payment agreements (305). All values not in U.S. dollars are to be converted to U.S. dollars at the exchange rates prevailing at end-June 2009 and then into cedis at the program exchange rate. A more detailed listing of accounts to be included in the measure of net foreign assets is contained in the monetary template referred to in paragraph 7 above.

17. Net international reserves of the BOG are defined for program monitoring purposes and in the balance of payments as short-term foreign assets of the BOG, minus short-term external liabilities. To the extent that short-term foreign assets are not fully convertible external assets readily available to and controlled by the BOG (that is, they are pledged or otherwise encumbered external assets, including, but not limited to, the HIPC umbrella SDR account), they will be excluded from the definition of net international reserves. Net international reserves are also defined to include net swap transactions (receivable less payable) and exclude all positive foreign currency deposits at the BOG held by resident deposit money banks, public institutions, nonfinancial public enterprises, other financial institutions, and the private sector. All values not in U.S. dollars are to be converted to U.S. dollars at the exchange rates prevailing at end-June 2009 and then into cedis at the program exchange rate. A more detailed listing of accounts to be included in the measure of net international reserves is contained in the monetary template referred to in the paragraph above.

18. Net domestic assets of the Bank of Ghana are defined as the difference between reserve money and net foreign assets of the BOG, excluding the HIPC Umbrella SDR account, converted from U.S. dollars to cedis at the program exchange rate.

\section{External Debt and Debt Service}

19. Short-term external debt is defined as the outstanding stock of external debt with an original maturity of one year or less, including overdraft positions and debt owed or guaranteed by the government or the BOG. ${ }^{1}$ Data on the BOG's short-term external debt are those reported from the statement of accounts template as short-term liabilities to nonresident

${ }^{1}$ (A) The term "debt" has the meaning set forth in point No. 9 of the Guidelines on Performance Criteria with Respect to Foreign Debt (Decision No. 12274-(00/85) August 24, 2000). This includes overdrafts on accounts with correspondent banks. (B) Excluded from this performance criterion are normal import-related credits, pre-export financing credits of public enterprises, cocoa loans collateralized by cocoa contracts, and individual leases with a value of less than US\$100,000. 
commercial banks (BOG statement of accounts code 1201 plus 301 overdrafts plus Crown Agent).

20. Nonconcessional medium- and long-term external debt is defined as outstanding stock of external debt contracted or guaranteed by the government or Bank of Ghana with an original maturity of more than one year. ${ }^{2}$ Medium- and long-term debt and its concessionality will be reported by the Aid and Debt Management Unit of the Ministry of Finance and Economic Planning, and will be measured in U.S. dollars at current exchange rates.

21. External payment arrears would accrue when undisputed interest or amortization payments of the government are not made within the terms of the contract, or in conformity with the terms for interim relief provided under the enhanced HIPC Initiative and the deferral agreed with the Paris Club on December 10, 2001.

\section{E. Adjustors to the Program Targets}

\section{Program's quantitative targets are subject to the following adjustors:}

- Overall fiscal deficit of the government: the deficit ceiling will be adjusted upward (downward) for higher (lower) than programmed project loans; downward (upward) by 50 percent of lower than programmed program loans (grants), up to a maximum adjustment of GH $\notin \$ 75$ million; and downward for higher than programmed program grants by full amount.

\footnotetext{
2 (A) This performance criterion applies not only to debt as defined in point No. 9 of the Guidelines on Performance Criteria with Respect to Foreign Debt (Decision No. 12274-(00/85) August 24, 2000) but also to commitments contracted or guaranteed for which value has not been received.

(B) Excluded from this performance criterion are: debts with a grant element equivalent to 35 percent or more, calculated using currency-specific discount rates based on OECD commercial interest reference rates; borrowing for specific high-return public infrastructure investment projects, subject to prior consultation with the Fund. For 2009/10, the ceiling is set at US\$300 million to allow for possible guarantees to the Ghana National Petroleum Company to facilitate its participation in the first phase of a program to exploit offshore gas deposits. The grant element of each loan will be assessed with regard to the terms of the loan provided by a foreign official entity in connection with the loan in question. Loans provided by a private entity will not be considered concessional unless accompanied by a grant or grant element provided by a foreign official entity, such as both components constitute an integrated financing package with a combined grant element equal to at least 35 percent.
} 
- Net international reserves of the Bank of Ghana: the floor will be adjusted upward for any new SDR allocation. The NIR floor will also be adjusted upward for any excess of budget grants and loans relative to the program baseline.

- Net domestic financing of the government: the ceiling will be adjusted upward by 50 percent of any shortfall in programmed program loans and grants, up to a maximum adjustment of GH $\not 75$ million. For higher than programmed loans and grants, the ceiling will be adjusted downward by full amount.

- Net domestic assets of the Bank of Ghana: the ceiling will be adjusted downward for any new SDR allocation.

\section{F. Provision of Data to the Fund}

23. Data with respect to the variables subject to performance criteria and indicative targets will be provided to Fund staff on a monthly basis with a lag of no more than eight weeks (except for select data for which the reporting lag is explicitly specified in Table 1). The authorities will transmit promptly to Fund staff any data revisions. For any information (and data) that is (are) relevant for assessing performance against program objectives but is (are) not specifically defined in this memorandum, the authorities will consult with Fund staff. 
Table 1. Ghana: Data to be Reported to the IMF

Fiscal data (to be provided by the MOFEP)

Central budget operations for revenues, expenditures and financing.

Functional breakdown by Ministry, Department, and Agency of expenditure authorizations, payment vouchers issued, payment vouchers liquidated, and arrears. This data will also identify poverty-related and expenditures financed through the HIPC Initiative, as well as the inflows and disbursements from the HIPC receiving and drawing accounts at the BOG.

Divestiture receipts received by the budget (in cedis and foreign exchange, net of divestiture transactions costs).

Monetary data (to be provided by the BOG)

Net domestic assets and net international reserves of the BOG.

Detailed balance sheet of the monetary authorities.

Monetary survey detailing the consolidated balance sheet of commercial banks using the new BSD2 Report Form.

Summary position of government committed and uncommitted accounts at BOG, as well as total financing from BOG. Accompanying table showing composition of other receipts and other expenditure.

Composition of banking system and nonbanking system net claims on government.

Debt registry showing structure and holders of domestic government debt, at face value and at discount. Similar table showing holders of treasury bills for open market operations.

Balance of Payments (to be provided by the BOG)

Export and import data on value, volume, and unit values, by major categories and other major balance of payments variables.

Foreign exchange cash flow.
Monthly, within six weeks of the end of each month.

Monthly, within six weeks of the end of each month.

Monthly, within six weeks of the end of each month.

Monthly, within two weeks of the end of each month.

Monthly, within four weeks of the end of each month.

Monthly, within six weeks of the end of each month.

Monthly, within four weeks of the end of each month.

Monthly, within four weeks of the end of each month.

Monthly, within four weeks of the end of each month.

Quarterly, with a maximum lag of two months. Monthly, within four weeks of the end of the month. 
Table 1. Ghana: Data to be Reported to the IMF (concluded)

\section{External debt and foreign assistance data (to be provided by MOFEP)}

Information on the concessionality of all new external loans contracted by the government or with a government guarantee.

For the coming quarter: (i) total debt service due by creditor, (ii) amount of HIPC Initiative relief on each transaction, and (iii) debt service paid and the transfers to the HIPC Initiative account by creditor for the previous month. Report should cover government and government-guaranteed debt (as defined in this document).

External debt and external debt service incurred by enterprises with government ownership above 50 percent, even if loans have not been explicitly guaranteed by the government.

Disbursements of grants and loans by creditor

Other data (to be provided by GSS)

Overall consumer price index.

National accounts by sector of production, in nominal and real terms.

Electricity pricing (to be provided by the Ministry of Energy)

Data on the tariff structure and the cost of producing electricity.

Petroleum pricing (to be provided by the Ministry of Energy)

(i) a breakdown of costs, including the ex-refinery price, duties, levies, and margins, for each of the individual petroleum products; and

(ii) the indicative maximum price approved in the bi-weekly review of petroleum pricing for each of the individual petroleum products.
Monthly, within four weeks of the end of each quarter.

Quarterly within four weeks of the end of each quarter.

Quarterly, within three weeks of the end of each quarter.

Quarterly, within four weeks of the end of each quarter.

Monthly, within two weeks of the end of each month.

Annual, within three months of the end of each year (switching to quarterly when they become available).

Quarterly, within four weeks of the end of each quarter.

Bi-weekly, within two days of the completion of the pricing review. 


\title{
INTERNATIONAL DEVELOPMENT ASSOCIATION \\ INTERNATIONAL MONETARY FUND
}

\author{
GHANA
}

\section{Joint IMF and World Bank Debt Sustainability Analysis}

\author{
Prepared by the staffs of the World Bank and \\ the International Monetary Fund \\ Approved by Michael Atingi-Ego and Dominique Desruelle (IMF) \\ and Carlos Alberto Braga and Sudhir Shetty (World Bank)
}

June 29, 2009

This debt sustainability analysis (DSA) updates that prepared for the 2008 Article IV consultation, discussions for which were completed in June 2008. ${ }^{1}$ The starting point for the DSA is less favorable than a year ago, reflecting the rise in the debt-to-GDP ratio between end-2007 and end-2008 on account of the large fiscal deficit in 2008. At the same time, notwithstanding the large fiscal deficit in 2008, the new government's goals for fiscal consolidation are more ambitious than those discussed a year ago. Further, there is now greater assurance that oil production will start in 2011, and this assumption is now included in the macroeconomic baseline. The combination of a more ambitious fiscal consolidation sustained over the medium to long term, together with stronger real GDP growth and higher export levels post-oil production all contribute to a more favorable DSA baseline than in 2008. Public sector net debt is projected to fall to less than 40 percent of GDP by the end of the projection period from 60-70 percent of GDP in 2008- 2012. By contrast, DSA baseline projections in 2008 showed public debt rising to exceed 80 percent of GDP over a 20 -year period. Notwithstanding this improvement, stress test analysis suggests that Ghana remains at moderate risk of debt distress, in line with the 2008 DSA.

Key risks to Ghana's debt sustainability relate to the medium- to long-term fiscal outlook, as well as the prospects for growth in a post-oil economy. The baseline depends on determined

\footnotetext{
${ }^{1}$ The DSA was prepared by IMF and World Bank staffs in collaboration with the Ghanaian authorities.
} 
fiscal adjustment in the near term, combined with rigorous efforts to limit borrowing needs over the medium- to long-term. Failure to reduce the primary deficit from 2009 levels would be associated with a near doubling of the public debt-to-GDP ratio over two decades. Similarly, the baseline assumes sustained, strong growth in the non-oil, non-mineral economy and a diversified and competitive export base. This will require macroeconomic stability, continued improvements in the business climate, and prudent management of Ghana's oil revenues.

\section{BACKGROUND}

1. Ghana's public debt at end-2008 was an estimated 58 percent of GDP. This compares with a projection of 51 percent of GDP in the 2008 DSA. The less favorable starting position for the current DSA reflects the larger than previously assumed fiscal deficit in 2008 (141/2 percent of GDP, or 4 percentage points higher than previously projected), as well as the impact of currency depreciation on the foreign debt-to-GDP ratio in 2008. Public sector external and domestic debts were similar in scale at end-2008, both close to US\$4 billion (29 percent of GDP each).

\section{External debt has risen rapidly since 2006 - up from 17 to 29 percent of GDP.} This reflects Ghana's $\$ 750$ million Eurobond issue at end-2007, together with new concessional bilateral financing, and new borrowing contracted from the IDA since 2006, following the Multilateral Debt Reduction Initiative (MDRI). Data on private sector external debt are of low quality, but appear to have remained broadly stable at about 15 percent of GDP between 2007 and 2008 .

\section{The sharp rise in Ghana's external (and total) public debt during 2006-08}

illustrates the risks to the DSA. A highly expansionary fiscal position financed by external borrowing triggered a very rapid deterioration in the debt position. This trend was amplified by the resulting balance of payments pressures and currency depreciation, which led to the revaluation of foreign currency-denominated claims relative to domestic GDP. This debt surge was effectively stemmed when Ghana's access to market financing was closed off as a result of the global financing crisis. Avoiding future such episodes of debt deterioration will require more determined fiscal management as well as more cautious debt management policies. 
Ghana: Total public Debt, 2004-08

\begin{tabular}{|c|c|c|c|c|c|}
\hline & 2004 & 2005 & 2006 & 2007 & 2008 \\
\hline & \multicolumn{5}{|c|}{ (In millions of U.S. dollars) } \\
\hline 1. External debt & 6,448 & 6,348 & 2,177 & 3,586 & 4,035 \\
\hline Multilateral Institutions & 5,287 & 5,565 & 1,327 & 1,710 & 2,028 \\
\hline IMF & 447 & 424 & 158 & 167 & 163 \\
\hline IDA & 4,012 & 4,336 & 803 & 1,137 & 1,320 \\
\hline AfDB & 551 & 555 & 141 & 153 & 230 \\
\hline Other & 277 & 251 & 225 & 254 & 315 \\
\hline Official bilateral & 960 & 636 & 760 & 978 & 1,168 \\
\hline Non-concessional $^{1}$ & 201 & 147 & 90 & 898 & 839 \\
\hline 2. Domestic debt & 1,868 & 1,997 & 3,133 & 3,819 & 4,020 \\
\hline Banking system & 1,402 & 1,755 & 2,431 & 2,598 & 2,588 \\
\hline Non-bank sector & 466 & 242 & 637 & 785 & 920 \\
\hline Non-residents & 0 & 0 & 66 & 437 & 363 \\
\hline Other $^{2}$ & 0 & 0 & 0 & 0 & 149 \\
\hline 3. Total public debt $(1+2)$ & 8,315 & 8,345 & 5,310 & 7,405 & 8,055 \\
\hline \multicolumn{6}{|l|}{ Memorandum items } \\
\hline Total public debt ${ }^{3}$ & 94.2 & 78.3 & 42.0 & 51.2 & 58.2 \\
\hline External debt & 73.1 & 59.6 & 17.2 & 24.8 & 29.2 \\
\hline Domestic debt & 21.2 & 18.8 & 24.8 & 26.4 & 29.0 \\
\hline
\end{tabular}

Source: Ministry of Finance and Bank of Ghana.

${ }^{1}$ Includes a bond placement in September 2007.

${ }^{2}$ Includes Jubilee bond and other standard credits.

${ }^{3}$ In percent of GDP.

\section{BASELINE SCENARIO}

\section{A. Fiscal and oil sector assumptions}

4. The baseline scenario features early correction of Ghana's large fiscal deficit. During 2009-10, the budget is strengthened by planned cuts in public spending, relative to GDP, based on a rationalization of capital spending, efforts to contain wage bill expansion, and the elimination of energy subsidies. At the same time, the revenue-to-GDP ratio is projected to rise, reflecting the planned elimination of customs tariff and other tax 
exemptions and rationalization of VAT thresholds and coverage. Moreover, government revenues are projected to receive a boost from the planned implementation of a single revenue authority for income, customs, and VAT tax collections. As a consequence, the fiscal deficit is projected to decline from 141/2 percent of GDP in 2008 to 6 percent in $2010 .^{2}$

\section{The fiscal accounts also benefit from 2011 onwards from Ghana's projected} move to oil producer status. This is now more assured than a year ago, even if a declaration of commercial viability remains to be issued (as of mid-June 2009). The DSA baseline reflects planned production from the Jubilee I offshore oil field, but does not include possible gas production or production from other oil fields for which exploration is still underway. Oil production is assumed to average 120 thousand barrels per day during 2012-15, with a subsequent steady decline. Based on the latest WEO projections, the price of Ghana's oil exports would increase to US $\$ 83$ per barrel by 2014; prices are assumed to stabilize at this level over the medium term in nominal terms. Oil revenues are projected to peak at about 6 percent of GDP during 2011-2017 (see Text Table). The government's incomes reflect a combination of income tax and royalty collections, production sharing agreements, and provision for an additional oil entitlement if favorable oil prices result in a rate of return in excess of specific thresholds. The latter element is tentatively projected to increase from under 1 percent of GDP during 2011-2018 to more than 1 1/2 percent of GDP during 2019-25.

6. The DSA is strengthened, temporarily, by projected oil revenue savings. The government is in the process of setting up a framework that would guide the utilization of oil resources. For purposes of this DSA, we assume that one-third of tax and royalty revenues would be saved, while two-thirds would be spent on growth-enhancing investment projects. ${ }^{3}$ For the additional oil entitlement, which is a less predictable source of revenue, the DSA assumes that one-half is saved, with the remainder spent, as above, on investment projects. Oil savings are projected to reach a cumulative 6 percent of GDP by 2017, effectively reducing public sector net indebtedness. ${ }^{4}$ The saved oil revenues would support spending over the long-term. When oil revenues begin a projected decline from 2019, spending would be sustained in part by drawing down the accumulated oil savings. The oil savings account would be depleted by 2028 and thereafter oil-related spending would be limited to about 1 percent of GDP, in line with residual oil revenues. Although the 2008 DSA baseline did not

\footnotetext{
${ }^{2}$ The 8.5 percent of GDP reduction in the fiscal deficit during 2008-2010 is projected to occur through a 1.1 percent of GDP increase in revenues, 3.8 percent of GDP decline in expenditures, 0.1 percent of GDP reduction in arrears, and an additional 3.5 percent of GDP in fiscal measures.

${ }^{3}$ These projects are expected to address the key bottlenecks in developing the industrial and manufacturing capacity of Ghana, including transportation infrastructure, telecommunication, power and energy, as well enhancing the productivity of agriculture. Project selections would be guided by adequate cost-benefit analysis yielding high rates of return.

${ }^{4}$ The DSA assumes that oil savings are invested abroad and earn a yield in line with the U.S. dollar Libor interest rate.
} 
include potential oil production, this possibility was examined in an alternative scenario. ${ }^{5}$ This scenario assumed that oil revenues would be saved throughout the DSA period, in contrast to the assumption here that the revenue stream would be fully spent by the end of the DSA. As a result, the impact of oil production in the 2008 alternative scenario was more positive than in the DSA baseline prepared for this report.

Long-Term Fiscal Baseline, 2009-2029

(In percent of GDP)

\begin{tabular}{|c|c|c|c|}
\hline & $\frac{\text { Pre-oil }}{(2009-2010)}$ & $\begin{array}{r}\text { Oil surplus } \\
\text { saved } \\
(2011-2018)\end{array}$ & $\begin{array}{r}\text { Oil savings } \\
\text { consumed } \\
(2019-2029)\end{array}$ \\
\hline Revenues and grants, of which: & 29.1 & 29.3 & 29.2 \\
\hline Non-oil revenues & 23.6 & 20.7 & 25.0 \\
\hline Oil revenues & 0.0 & 5.7 & 2.7 \\
\hline Of which: additional oil entitlement & 0.0 & 0.8 & 1.4 \\
\hline Grants & 5.5 & 2.9 & 1.5 \\
\hline Expenditures, of which: & 37.2 & 33.0 & 33.3 \\
\hline Oil-financed projects & 0.0 & 3.8 & 3.4 \\
\hline Other fiscal items, net ${ }^{1}$ & -0.4 & -1.8 & -2.1 \\
\hline Overall balance & -7.7 & -1.9 & -2.0 \\
\hline \multicolumn{4}{|l|}{ Memorandum items: } \\
\hline Non-oil balance & -7.7 & -3.7 & -1.3 \\
\hline Primary balance & -3.8 & 0.2 & 0.1 \\
\hline Net public debt (end-period) & 66.6 & 44.2 & 36.1 \\
\hline Gross public debt & 66.6 & 57.0 & 36.1 \\
\hline Oil savings balance (-) & 0.0 & 12.8 & 0.0 \\
\hline
\end{tabular}

${ }^{1}$ Arrears clearance, VAT refunds, and new fiscal measures in 2010 budget.

\section{B. Macroeconomic assumptions}

7. The DSA baseline assumes prudent economic policies to foster stable macroeconomic conditions. A decline in inflation to single-digit levels and strong productivity growth would support broad-based growth in the non-extractive sectors (Box 1).

\footnotetext{
${ }^{5}$ See Figure 4 of the 2008 DSA.
} 


\section{Box 1: Baseline Macroeconomic Assumptions}

Real GDP growth: After averaging more than 6 percent annually during 2004-08, output growth is set to slow to below 5 percent during 2009-10 reflecting fiscal tightening and global slowdown. Real GDP would expand by about 20 percent in 2011 as oil production begins. In the nonoil sector, growth is projected to average 5-6 percent during 2012-29, reflecting broad-based non-mineral growth.

Inflation: Consumer price inflation exceeded 20 percent in the first quarter of 2009. A decline through 2009-2010 reflects slowing economic growth, fiscal tightening, and tight monetary policies. Over the medium term, inflation is expected to converge to the Bank of Ghana's target of 5 percent.

Government balances: The primary deficit is assumed to narrow to less than one percent of GDP over the long-term. Expenditures would remain broadly stable in relation to the GDP, with a reprioritization toward growth-oriented infrastructure investments financed by oil revenues. Foreign grant receipts are projected to decline relative to GDP as Ghana benefits from oil incomes, but this is more than offset by an increase in domestic revenues from non-oil taxation.

Current account balance: Over 2009-11, the current account deficit is projected to narrow but remain at double-digit level in relation to GDP, reflecting imported equipments for the new oil fields as well as projected government's infrastructure investment. Beyond that, the current account deficit would narrow to about 4-5 percent of GDP by the end of the forecasting period. This assumption is consistent with fundamental determinants of Ghana's domestic savings and investment, as explained in Box 2. Exports are projected to peak at 62.3 percent of GDP during 2012, boosted by oil production. During 2019-2029, declining oil exports are partly offset by strong growth in non-mineral exports, leaving exports at 47.4 percent of GDP in 2029.

Financing flows. Ghana's deficit in trade in goods and services is projected to remain in the 1416 percent of GDP range. This would be financed, in large part, by private and public transfers averaging 14-15 percent of GDP, with a gradual decline in official transfers being offset by higher private transfers. Non-debt creating inflows (mainly comprising foreign direct investment) are projected to average about 10 percent of GDP during 2009-14, largely reflecting development of the oil and gas sector. The growth of the nonoil economy, including the expansion of services, is expected to attract foreign investment. As oil activity wanes, these inflows would gradually decline to 4-5 percent of GDP during the medium-tolong run, in line with the average for Sub-Saharan African countries. 


\section{Box 2: Fundamental Determinants of Ghana's Current Account Balance Norm}

Economic theory suggests that the long-term external balance is influenced by the key fundamental determinants of domestic savings and investments. Below parameter estimates from a panel study are used to project Ghana's long-term current account deficit. ${ }^{1}$

Fiscal balance (rel. to trading partners)

Population growth rate (rel. to trading partners) 1/ Initial NFA (from Lane and Milesi-Ferretti)

Oil balance to GDP (average 2005-08, WEO)

Per capita growth (rel. to trading partners)

Current account norm
The Macroeconomic Balance Approach (dependent variable CA/GDP)

\begin{tabular}{rrrrrr}
\multirow{2}{*}{$\begin{array}{c}\text { Pooled } \\
\text { parameter }\end{array}$} & \multicolumn{2}{c}{ Ghana } & \multicolumn{2}{c}{ Trading partners } & \multicolumn{2}{c}{ Pooled results } \\
\cline { 2 - 6 } & $2011-18$ & $2019-25$ & (four-year averages) & $2011-18$ & $2019-25$ \\
\hline 0.20 & -3.2 & -3.3 & -1.8 & -0.3 & -0.3 \\
-1.21 & 1.6 & 1.1 & 0.6 & -1.1 & -0.6 \\
0.02 & -79.0 & -79.0 & & -1.6 & -1.6 \\
0.23 & -2.0 & -8.4 & \multirow{2}{*}{1.4} & -0.5 & -1.9 \\
-0.21 & 4.5 & 2.7 & -0.6 & -0.3 \\
\hline & & & -4.1 & $\mathbf{- 4 . 7}$
\end{tabular}

The above analysis suggests a current account deficit of about 4-5 percent of GDP in the medium-to-long-term. For comparison, the estimate for current account deficit norm in 2009 using the same methodology yielded 8.6 percent of GDP. The difference reflects a smaller fiscal deficit, slowing population growth, and a smaller oil import deficit.

${ }^{1}$ See Jaewoo Lee et al., "Exchange Rate Assessments: CGER Methodologies," Occasional Paper, No. 261, IMF, Washington DC, 2008.

\section{External Debt Sustainability}

\section{The baseline displays stable external debt indicators, which remain well below} the respective thresholds (Figure 1). ${ }^{6}$ These results show substantial improvement over the 2008 DSA where the external debt indicators gradually deteriorated over the medium-to-long run, although remaining below the respective thresholds. This substantial improvement partly reflects the inclusion of oil in the baseline scenario, which has the effect of increasing output and export levels.

\section{The public sector external debt-to-GDP ratio is projected to rise modestly} between 2008 and 2029 (from 29 to 33 percent of GDP). The increase would be somewhat larger in net present value terms (from 18 to 31 percent of GDP), reflecting an assumed increase in nonconcessional borrowing. The NPV of private external debt is projected to decline from 13.7 percent of GDP in 2008 to below 10 percent by 2029, as external financing would be increasingly in the form of non-debt creating direct investment inflows (Table 1).

\footnotetext{
${ }^{6}$ The World Bank's Country Policy and Institutional Assessment (CPIA) rates Ghana as a strong performer. Under the joint IMF-World Bank debt sustainability framework, the corresponding indicative debt burden thresholds are 50 percent for the NPV of debt-to-GDP ratio, 200 percent for the NPV of debt-to-exports ratio, and 25 percent for the debt-service-to-exports ratio. See Operational Framework for Debt Sustainability Framework in Low-Income Countries-Further Consideration, available at www.imf.org and www.worldbank.org.
} 
10. Increased borrowing at non-concessional terms raises the cost of servicing debt. As Ghana becomes more prosperous and moves to middle-income status, the structure of the country's external financing is likely to change. In particular, reduced grant and concessional financing would give way to borrowing at non-concessional terms, which is typically at shorter maturity and higher cost (in the DSA, the average maturity of non-concessional borrowing is 6 years and the assumed interest rate is 8 percent). A shift to non-concessional funding would leave the debt-to-GDP ratio largely unaffected, while increasing the debt service ratios (measured relative to exports and government revenues, see Figure 1). The DSA assumes a high-case level of non-concessional borrowing of US $\$ 0.5$ billion annually during 2009-14. ${ }^{7}$ While this overstates current non-concessional borrowing plans, it ensures that the DSA is resilient to higher outturns. This approach also covers the risks that nonconcessional borrowing will adversely affect the grant element of new IDA financing.

\section{Stress Testing and Alternative Scenarios}

\section{In the case of Ghana, standardized stress tests potentially overstate DSA risks.}

The standard stress tests for growth and export performance assume that outturns during 2010-2011 fall short of the historic average by one standard deviation. This represents a dramatic revision to baseline projections for 2011, where growth and exports are both projected to surge with the start of oil production. ${ }^{8}$ Given the step-like profile of oil production and exports - with a one-off increase in 2011 followed by broad stability for the following five-year period, these stress tests effectively eliminate the benefits of the oil sector from the DSA baseline. Indeed, the outcome is worse than a scenario without oil, since oil exports are eliminated without reducing the imports that they would have financed.

\section{The DSA is much more resilient when standardized tests are adjusted to reflect} the arrival of oil in 2011. We applied the standardized shocks to the period 2009-10, yet retaining the oil-induced acceleration in real growth and exports starting in 2011 (Figure 1 and Table 2). In these stress tests, all debt indicators would remain below their respective thresholds, except for the debt service-to-revenue ratio, which would exceed the threshold level by a modest amount toward the end of the projection period.

\footnotetext{
${ }^{7}$ The PRGF provides for a maximum of US $\$ 300$ million of new non-concessional borrowing through mid-2010 to finance oil and gas projects. This explains the difference in external debt-to-GDP figures with the staff report.

${ }^{8}$ Real GDP is projected to rise by 24 percent in 2011, while exports are projected to rise by 51 percent in dollar terms.
} 


\section{E. Public Debt Sustainability}

\section{The baseline scenario shows that Ghana's net public debt would fall during the} medium term from 73 percent of GDP in 2010 to 37 percent by 2029. ${ }^{9}$ This would return the debt ratio to below the recent post-MDRI low of 41 percent in 2006. The projected decline in debt-to-GDP ratio reflects a move to broad primary fiscal balance, which limits new debt creation, combined with sustained economic expansion (Table 3).

14. The DSA benefits from a projected decline in domestic borrowing. In the baseline scenario, the present value of debt-to-GDP ratio benefits from a rebalancing from domestic to foreign debt, which has higher concessionality. A more balanced reduction in foreign and domestic debt would be associated with a less marked improvement in the present value of debt-to-GDP ratio and the debt service indicators. At the same time, a larger reduction in foreign debt would reduce vulnerability to exchange rate changes.

15. The favorable DSA depends on successful fiscal stabilization. If the primary balance remains at projected 2009 levels (5 percent of GDP) throughout the DSA, the present value of debt would rise from 59 to 109 percent of GDP. The DSA is also sensitive to currency valuation, with a 30 percent depreciation raising the present value of debt-to-GDP ratio by 25 percentage points by the end of the projection period.

16. The DSA is relatively insensitive to oil price assumptions. Alternative scenarios to explore the impact of different oil price assumptions produced little net change in the DSA baseline, though less favorable results are possible, depending on the assumptions. In a straightforward balance of payments projection, a lower global oil price would result in deterioration in the trade balance during 2011-15, when Ghana is a net oil exporter. But the trade balance would strengthen in the subsequent period, when Ghana shifts back to net oil importer status. As a result, Ghana's resort to additional external financing is limited in duration (2011-15), and offset by improvements in the balance of payments beyond 2015. At the level of the public sector, the DSA impact depends on how spending responds to lower oil revenues. The DSA baseline assumes that spending moves in line with oil revenues over the long term, constrained by the assumption that oil-related spending slows once the oil fund is depleted. If the government sought to maintain spending at higher levels, even in the absence of the necessary oil fund savings, the DSA would be less favorable.

\footnotetext{
${ }^{9}$ The higher level compared to the IMF staff report figures reflect the additional nonconcessional borrowing included in the DSA analysis (para. 10).
} 


\section{Conclusions}

17. The 2009 DSA shows an improvement from last year under the baseline for key debt indicators. This reflects a tighter fiscal stance over the DSA period than in last year's DSA, combined with the incorporation of oil production in the baseline for the first time. Debt ratios are projected to be broadly stable over the DSA period, rather than showing the deteriorating trend evident in the 2008 baseline. Moreover, while the debt service indicators rise, the trend is more favorable than in last year's baseline. The stress test results are generally favorable, once allowance is made for complications in applying standardized tests to the period in which oil production and exports are projected to start. As discussed in paragraph 12, the most extreme shocks are projected to leave the debt indicators well below threshold levels except for the debt service-to-revenue ratio, which would slightly exceed the 35 percent threshold.

\section{Despite the baseline improvements, Ghana remains at moderate risk of debt} distress due to short- and medium-term vulnerabilities from two key factors: oil revenues and fiscal performance. Even with oil production, failure to reduce the large primary fiscal deficit and sustain this consolidation over the coming years would result in a much less favorable DSA outlook. The historic scenario, which reflects the looser fiscal stance in recent years, shows substantially higher debt-to-GDP and debt-service ratios, with the former quickly exceeding the threshold levels. Therefore, the ability to sustain fiscal consolidation over the medium to long term, and the potential to accelerate non-oil growth in the medium term while oil revenues increase have emerged as key risks to an otherwise favorable baseline.

\section{The DSA depends closely on prudent macroeconomic management of oil wealth.}

The baseline assumes that oil production is combined with strong private sector investment and sustained strong growth in the non-mineral sector, and continued export diversification. This will require judicious use of oil revenues in terms of the size and nature of additional public expenditure to limit Dutch disease effects. If the latter were to emerge as a problem, the DSA would be adversely impacted. The new oil fields currently under exploration could further improve the fiscal and growth performance in the baseline-though the premium on prudent management of oil revenues would be redoubled. To minimize these risks, it will be important to develop a strategy for using oil and gas revenues in a productive manner. 
Table 1. Ghana.: External Debt Sustainability Framework, Baseline Scenario, 2006-2029 1/

(nn percent of GDP, unless otherwise indicated)

\begin{tabular}{|c|c|c|c|c|c|c|c|c|c|c|c|c|c|c|c|}
\hline & \multicolumn{3}{|c|}{ Actual } & \multirow{2}{*}{$\begin{array}{c}\text { Historical } \\
\text { Average }\end{array}$} & \multirow{2}{*}{$\begin{array}{l}\text { Standard } \\
\text { Deviation }\end{array}$} & \multicolumn{6}{|c|}{ Projections } & \multirow[b]{2}{*}{$\begin{array}{c}2009-2014 \\
\text { Average }\end{array}$} & \multirow[b]{2}{*}{2019} & \multirow[b]{2}{*}{2029} & \multirow[b]{2}{*}{$\begin{array}{c}\text { 2015-2029 } \\
\text { Average }\end{array}$} \\
\hline & 2006 & 2007 & 2008 & & & 2009 & 2010 & 2011 & 2012 & 2013 & 2014 & & & & \\
\hline External debt (nominal) 1/ & 33.3 & 39.2 & 42.8 & 94.4 & 46.5 & 57.2 & 63.4 & 57.3 & 56.8 & 55.8 & $\mathbf{5 3 . 5}$ & 57.3 & 49.1 & 40.2 & 46.6 \\
\hline $\mathrm{o} / \mathrm{w}$ public and publicly guaranteed (PPG) & 17.2 & 24.8 & 29.2 & 79.7 & 46.4 & 41.5 & 47.7 & 44.1 & 43.9 & 43.3 & 41.4 & 43.7 & 38.5 & 33.5 & 37.2 \\
\hline Change in external debt & -41.6 & 5.9 & 3.6 & -5.1 & 27.6 & 14.4 & 6.2 & -6.1 & -0.4 & -1.0 & -2.3 & 1.8 & 0.1 & -1.2 & -0.9 \\
\hline Identified net debt-creating flows & -6.8 & 1.3 & 9.1 & 1.8 & 27.7 & 3.1 & 0.8 & -14.4 & -4.9 & -3.6 & -3.5 & -3.8 & 1.5 & -1.1 & 0.1 \\
\hline Non-interest current account deficit & 9.2 & 11.7 & 18.5 & 6.2 & 6.3 & 11.8 & 14.7 & 8.3 & 7.1 & 8.3 & 4.9 & 9.2 & 8.4 & 2.4 & 6.5 \\
\hline Deficit in balance of goods and services & 24.9 & 27.1 & 34.2 & 21.4 & 6.6 & 26.3 & 28.8 & 13.8 & 12.3 & 13.9 & 11.2 & 17.7 & 19.4 & 15.4 & 17.9 \\
\hline Exports & 40.2 & 40.1 & 44.0 & 40.9 & 4.6 & 52.2 & 54.4 & 61.8 & 62.3 & 60.7 & 59.2 & 58.4 & 52.1 & 47.4 & 51.0 \\
\hline Imports & 65.1 & 67.2 & 78.1 & 62.3 & 8.1 & 78.4 & 83.2 & 75.6 & 74.7 & 74.6 & 70.3 & 76.1 & 71.5 & 62.8 & 68.9 \\
\hline Net current transfers (negative $=$ inflow) & -16.0 & -16.0 & -16.5 & -15.2 & 3.2 & -14.5 & -14.0 & -13.5 & -14.0 & -14.4 & -14.9 & -14.2 & -14.0 & -13.3 & -13.8 \\
\hline $\mathrm{o} / \mathrm{w}$ official & -3.1 & -3.7 & -4.2 & -3.7 & 1.0 & -5.0 & -4.0 & -2.5 & -2.5 & -2.4 & -2.4 & -3.1 & -1.0 & -0.3 & -0.8 \\
\hline Other current account flows (negative $=$ net inflow) & 0.3 & 0.6 & 0.8 & 0.4 & 0.4 & 0.0 & -0.1 & 7.9 & 8.8 & 8.8 & 8.6 & 5.7 & 3.1 & 0.3 & 2.4 \\
\hline Net FDI (negative $=$ inflow) & -5.0 & -5.7 & -7.5 & -2.5 & 2.5 & -7.9 & -12.4 & -11.5 & -9.4 & -10.4 & -7.0 & -9.7 & -6.0 & -3.0 & -5.7 \\
\hline Endogenous debt dynamics 2 / & -11.1 & -4.7 & -1.8 & -2.3 & 26.2 & -0.8 & -1.5 & -11.2 & -2.6 & -1.6 & -1.4 & -3.2 & -0.8 & -0.5 & -0.8 \\
\hline Contribution from nominal interest rate & 0.7 & 0.3 & 0.9 & 1.6 & 0.8 & 1.4 & 1.2 & 1.2 & 1.3 & 1.2 & 1.2 & 1.3 & 1.0 & 1.5 & 1.3 \\
\hline Contribution from real GDP growth & -4.1 & -1.6 & -2.7 & -4.6 & 1.7 & -2.2 & -2.8 & -12.4 & -3.9 & -2.8 & -2.6 & -4.4 & -1.9 & -2.0 & -2.0 \\
\hline Contribution from price and exchange rate changes & -7.7 & -3.4 & 0.0 & 0.7 & 26.4 & & & $0-12.7$ & 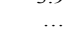 & 2.0 & 2.0 & & -1.3 & -2.0 & \\
\hline Residual (3-4) 3/ & -34.7 & 4.6 & -5.6 & -6.9 & 19.4 & 11.2 & 5.4 & 8.3 & 4.5 & 2.6 & 1.2 & 5.5 & -1.4 & -0.1 & -0.9 \\
\hline $\mathrm{o} / \mathrm{w}$ exceptional financing & 0.0 & 0.0 & 0.0 & 2.2 & 2.6 & 1.7 & 0.8 & 0.0 & 0.0 & 0.0 & 0.0 & 0.4 & 0.0 & 0.0 & 0.0 \\
\hline PV of external debt $4 /$ & $\ldots$ & $\ldots$ & 31.7 & 31.7 & & 43.8 & 49.6 & 45.0 & 45.3 & 44.4 & 42.5 & 45.1 & 40.4 & 38.2 & 40.0 \\
\hline In percent of exports & $\ldots$ & $\ldots$ & 72.0 & 72.0 & & 84.0 & 91.1 & 72.7 & 72.6 & 73.2 & 71.8 & 77.6 & 77.6 & 80.6 & 78.7 \\
\hline PV of PPG external debt & $\ldots$ & ... & 18.0 & 18.0 & & 28.2 & 33.9 & 31.8 & 32.3 & 31.9 & 30.4 & 31.4 & 29.8 & 31.4 & 30.6 \\
\hline In percent of exports & $\ldots$ & $\ldots$ & 41.0 & 41.0 & & 54.0 & 62.3 & 51.4 & 51.9 & 52.6 & 51.4 & 53.9 & 57.2 & 66.2 & 60.3 \\
\hline In percent of government revenues & $\ldots$ & $\ldots$ & 79.0 & 79.0 & & 117.0 & 138.6 & 121.9 & 123.0 & 121.8 & 117.6 & 123.3 & 105.6 & 114.4 & 110.1 \\
\hline Debt service-to-exports ratio (in percent) & 17.8 & 9.8 & 10.8 & 20.9 & 6.4 & 9.9 & 10.4 & 8.7 & 9.4 & 9.9 & 10.7 & 9.8 & 12.4 & 18.4 & 15.1 \\
\hline PPG debt service-to-exports ratio (in percent) & 11.4 & 3.0 & 4.6 & 14.2 & 6.7 & 4.0 & 4.8 & 4.6 & 5.4 & 5.9 & 6.8 & 5.3 & 8.5 & 15.6 & 11.5 \\
\hline PPG debt service-to-revenue ratio (in percent) & 20.8 & 5.3 & 8.9 & 32.3 & 19.9 & 8.7 & 10.8 & 10.9 & 12.9 & 13.7 & 15.6 & 12.1 & 15.7 & 27.0 & 20.9 \\
\hline Total gross financing need (Billions of U.S. dollars) & 1.4 & 1.5 & 2.5 & 1.3 & 0.7 & 1.5 & 1.2 & 0.4 & 0.7 & 0.9 & 1.0 & 0.9 & 2.8 & 5.9 & 3.6 \\
\hline Non-interest current account deficit that stabilizes debt ratio & 50.8 & 5.8 & 14.9 & 11.7 & 26.4 & -2.6 & 8.5 & 14.4 & 7.5 & 9.3 & 7.2 & 7.4 & 8.3 & 3.6 & 7.4 \\
\hline \multicolumn{16}{|l|}{ Key macroeconomic assumptions } \\
\hline Real GDP growth (in percent) & 6.4 & 5.7 & 7.3 & 5.3 & 1.1 & 4.5 & 5.0 & 24.2 & 7.1 & 5.3 & 5.0 & 8.5 & 4.1 & 5.4 & 4.6 \\
\hline GDP deflator in US dollar terms (change in percent) & 11.5 & 11.4 & 0.1 & 3.9 & 15.8 & -14.4 & -1.5 & -0.8 & -1.0 & 2.1 & 2.7 & -2.2 & 2.9 & 4.7 & 3.3 \\
\hline Effective interest rate (percent) $5 /$ & 1.1 & 1.1 & 2.4 & 1.7 & 0.4 & 2.9 & 2.2 & 2.4 & 2.3 & 2.3 & 2.3 & 2.4 & 2.3 & 4.1 & 2.9 \\
\hline Growth of exports of G\&S (US dollar terms, in percent) & 30.7 & 17.5 & 17.8 & 11.3 & 10.7 & 6.2 & 7.8 & 40.0 & 6.9 & 4.6 & 5.2 & 11.8 & 4.7 & 8.9 & 6.5 \\
\hline Growth of imports of G\&S (US dollar terms, in percent) & 25.2 & 21.5 & 24.9 & 14.5 & 14.1 & -10.2 & 9.6 & 12.0 & 4.7 & 7.3 & 1.7 & 4.2 & 6.9 & 7.5 & 7.2 \\
\hline Grant element of new public sector borrowing (in percent) & & & & & & 22.5 & 22.0 & 20.4 & 19.6 & 17.2 & 18.7 & 20.1 & 4.4 & -5.6 & 1.8 \\
\hline Government revenues (excluding grants, in percent of GDP) & 21.9 & 22.7 & 22.8 & 19.7 & 3.5 & 24.1 & 24.4 & 26.1 & 26.3 & 26.2 & 25.8 & 25.5 & 28.2 & 27.4 & 27.8 \\
\hline Aid flows (in Billions of US dollars) 7/ & 0.7 & 0.9 & 0.7 & 0.4 & 0.3 & 1.5 & 1.3 & 1.2 & 1.0 & 1.1 & 1.1 & 1.2 & 1.1 & 1.3 & 1.1 \\
\hline o/w Grants & 0.7 & 0.9 & 0.7 & 0.4 & 0.3 & 0.9 & 0.7 & 0.6 & 0.6 & 0.7 & 0.7 & 0.7 & 0.6 & 0.9 & 0.7 \\
\hline $\mathrm{o} / \mathrm{w}$ Concessional loans & 0.0 & 0.0 & 0.0 & 0.0 & 0.0 & 0.6 & 0.6 & 0.6 & 0.4 & 0.4 & 0.4 & 0.5 & 0.4 & 0.4 & 0.4 \\
\hline Grant-equivalent financing (in percent of GDP) $8 /$ & $\ldots$ & $\ldots$ & $\ldots$ & & & 8.6 & 7.2 & 4.8 & 4.4 & 4.1 & 4.1 & 5.5 & 2.3 & 0.8 & 1.8 \\
\hline Grant-equivalent financing (in percent of external financing) $8 /$ & $\ldots$ & & & & & 49.5 & 47.8 & 45.3 & 50.9 & 51.2 & 54.7 & 49.9 & 27.1 & 8.3 & 22.1 \\
\hline \multicolumn{16}{|l|}{ Memorandum items: } \\
\hline Nominal GDP (Billions of US dollars) & 12.7 & 15.0 & 16.1 & 9.5 & 4.0 & 14.4 & 14.9 & 18.3 & 19.4 & 20.9 & 22.5 & 18.4 & 30.9 & 72.0 & 42.3 \\
\hline Nominal dollar GDP growth & 18.7 & 17.7 & 7.3 & 9.5 & 17.1 & -10.6 & 3.4 & 23.2 & 6.0 & 7.5 & 7.8 & 6.2 & 7.1 & 10.4 & 8.1 \\
\hline PV of PPG external debt (in Billions of US dollars) & & & 2.9 & 2.9 & & 4.1 & 5.0 & 5.8 & 6.3 & 6.7 & 6.8 & 5.8 & 9.2 & 22.6 & 13.1 \\
\hline (PVt-PVt-1)/GDPt-1 (in percent) & & & & & & 7.2 & 6.8 & 5.3 & 2.5 & 1.9 & 0.9 & 4.1 & 3.2 & 3.1 & 2.6 \\
\hline
\end{tabular}

$1 /$ Includes both public and private sector external debt.

2/Derived a $[\mathrm{r}-\mathrm{g}-\mathrm{r}(1+\mathrm{g})] /(1+\mathrm{g}+\mathrm{r}+\mathrm{gr})$ times previous period debt ratio, with $\mathrm{r}=$ nominal interest rate; $\mathrm{g}=$ real GDP growth rate, and $\mathrm{r}=$ growth rate of GDP deflator in U.S. dollar terms.

3/ Includes exceptional financing (i.e., changes in arrears and debt relief); changes in gross foreign assets; and valuation adjustments. For projections also includes contribution from price and exchange rate changes.

5/ Assumes that PV of private sector debt is equivalent to its face value.

6/ Historical averages and standard deviations are generally derived over the past 10 years, subject to data availability.

8/ Grant-equivalent financing includes grants provided directly to the government and through new borrowing (difference between the face value and the PV of new debt). 


\begin{tabular}{|c|c|c|c|c|c|c|c|c|}
\hline & \multicolumn{8}{|c|}{ Projections } \\
\hline & 2009 & 2010 & 2011 & 2012 & 2013 & 2014 & 2019 & 2029 \\
\hline \multicolumn{9}{|c|}{ PV of debt-to GDP ratio } \\
\hline Baseline & 28 & 34 & 32 & 32.3 & 31.9 & 30.4 & 29.8 & 31 \\
\hline \multicolumn{9}{|l|}{ A. Alternative Scenarios } \\
\hline A1. Key variables at their historical averages in 2009-2029 1/ & 28 & 34 & 43 & 49 & 53 & 56 & 54 & 57 \\
\hline A2. New public sector loans on less favorable terms in 2009-2029 2 & 28 & 36 & 36 & 38 & 38 & 37 & 42 & 49 \\
\hline \multicolumn{9}{|l|}{ B. Bound Tests } \\
\hline B1. Real GDP growth at historic average minus one standard deviation in $2009-10$ & 28 & 34 & 32 & 32 & 32 & 30 & 30 & 31 \\
\hline B2. Export value growth at historic average minus one standard deviation in 2009-10 3/ & 28 & 40 & 45 & 45 & 44 & 41 & 35 & 32 \\
\hline B3. US dollar GDP deflator at historic average minus one standard deviation in 2009-10 & 28 & 38 & 35 & 36 & 35 & 34 & 33 & 35 \\
\hline B4. Net non-debt-creating flows at historic average minus one standard deviation in 2009-10 4/ & 28 & 49 & 45 & 45 & 43 & 40 & 34 & 32 \\
\hline B5. Combination of B1-B4 using one-half standard deviation shocks & 28 & 49 & 46 & 46 & 45 & 42 & 36 & 33 \\
\hline B6. One-time 30 percent nominal depreciation relative to the baseline in 20105 / & 28 & 49 & 46 & 46 & 46 & 43 & 43 & 45 \\
\hline
\end{tabular}

PV of debt-to-exports ratio

Baseline

A. Alternative Scenarios

A1. Key variables at their historical averages in 2009-2029 1/

A2. New public sector loans on less favorable terms in 2009-2029 2

B. Bound Tests

B1. Real GDP growth at historic average minus one standard deviation in 2009-10 B2. Export value growth at historic average minus one standard deviation in 2009-10 3 /

B3. US dollar GDP deflator at historic average minus one standard deviation in 2009-10

B4. Net non-debt-creating flows at historic average minus one standard deviation in 2009-10 4

B5. Combination of B1-B4 using one-half standard deviation shocks

B6. One-time 30 percent nominal depreciation relative to the baseline in 20105 /

PV of debt-to-revenue ratio

Baseline

117

139

117

A1. Key variables at their historical averages in 2009-2029 1/

A2. New public sector loans on less favorable terms in 2009-2029 2

B. Bound Tests

B1. Real GDP growth at historic average minus one standard deviation in 2009-10

B2. Export value growth at historic average minus one standard deviation in 2009-10 3 /

B3. US dollar GDP deflator at historic average minus one standard deviation in 2009-10

B4. Net non-debt-creating flows at historic average minus one standard deviation in 2009-10 4/

B5. Combination of B1-B4 using one-half standard deviation shocks

B6. One-time 30 percent nominal depreciation relative to the baseline in 20105 /
54

54

54

63

63

70
58

58

60

88

63

63

$103 \quad 121$

$\begin{array}{llllllll}54 & 62 & 51 & 52 & 52 & 51 & \mathbf{5 7} & 66 \\ 54 & 84 & 82 & 82 & 82 & 79 & \mathbf{7 6} & 76 \\ 54 & 62 & 51 & 52 & 52 & 51 & \mathbf{5 7} & 66 \\ 54 & 90 & 72 & 72 & 71 & 68 & \mathbf{6 6} & 67 \\ 54 & 89 & 73 & 74 & 73 & 70 & \mathbf{6 8} & 68 \\ 54 & 62 & 51 & 52 & 52 & 51 & \mathbf{5 7} & 66\end{array}$

$\begin{array}{llllllll}117 & 139 & 122 & 123 & 122 & 118 & \mathbf{1 0 6} & 115 \\ 117 & 165 & 172 & 172 & 168 & 160 & \mathbf{1 2 5} & 116 \\ 117 & 155 & 136 & 137 & 135 & 131 & \mathbf{1 1 7} & 127 \\ 117 & 200 & 171 & 171 & 165 & 156 & \mathbf{1 2 2} & 116 \\ 117 & 199 & 175 & 176 & 170 & 161 & \mathbf{1 2 6} & 119 \\ 117 & 199 & 175 & 176 & 174 & 168 & \mathbf{1 5 1} & 164\end{array}$


Table 2.Ghana: Sensitivity Analysis for Key Indicators of Public and Publicly Guaranteed External Debt, 2009-2029 (continued) (In percent)

\begin{tabular}{|c|c|c|c|c|c|c|c|c|}
\hline & \multicolumn{8}{|c|}{ Projections } \\
\hline & 2009 & 2010 & 2011 & 2012 & 2013 & 2014 & 2019 & 2029 \\
\hline
\end{tabular}

\section{Baselin}

A. Alternative Scenarios

A1. Key variables at their historical averages in 2009-2029 1/

A2. New public sector loans on less favorable terms in 2009-2029 2

\section{B. Bound Tests}

B1. Real GDP growth at historic average minus one standard deviation in 2009-10 B2. Export value growth at historic average minus one standard deviation in 2009-10 3/

B3. US dollar GDP deflator at historic average minus one standard deviation in 2009-10

B4. Net non-debt-creating flows at historic average minus one standard deviation in 2009-10 4/

B5. Combination of B1-B4 using one-half standard deviation shocks

B6. One-time 30 percent nominal depreciation relative to the baseline in 20105 /

$\begin{array}{lllllllll}4 & 5 & 5 & 5.4 & 5.9 & 6.8 & \mathbf{8 . 5} & 16\end{array}$

$\begin{array}{rrrrrrrr}4 & 5 & 5 & 6 & 7 & 10 & \mathbf{1 5} & 21 \\ 4 & 5 & 3 & 3 & 3 & 3 & \mathbf{6} & 21\end{array}$

Debt service-to-revenue ratio

\section{A. Alternative Scenarios}

A1. Key variables at their historical averages in 2009-2029 1/

A2. New public sector loans on less favorable terms in 2009-2029 2

$\begin{array}{rrrrrrrr}9 & 10 & 12 & 15 & 17 & 22 & \mathbf{2 8} & 36 \\ 9 & 11 & 6 & 7 & 7 & 8 & \mathbf{1 1} & 37\end{array}$

\section{B. Bound Tests}

B1. Real GDP growth at historic average minus one standard deviation in 2009-10

B2. Export value growth at historic average minus one standard deviation in 2009-10 3 /

B3. US dollar GDP deflator at historic average minus one standard deviation in 2009-10

B4. Net non-debt-creating flows at historic average minus one standard deviation in 2009-10 4

B5. Combination of B1-B4 using one-half standard deviation shocks

B6. One-time 30 percent nominal depreciation relative to the baseline in $20105 /$

Memorandum item

Grant element assumed on residual financing (i.e., financing required above baseline) 6

$\begin{array}{llllllll}9 & 11 & 11 & 13 & 14 & 16 & \mathbf{1 6} & 27 \\ 9 & 11 & 12 & 15 & 18 & 22 & \mathbf{2 0} & 27 \\ 9 & 12 & 12 & 14 & 15 & 17 & \mathbf{1 7} & 30 \\ 9 & 11 & 13 & 15 & 20 & 22 & \mathbf{2 0} & 27 \\ 9 & 11 & 14 & 16 & 20 & 22 & \mathbf{2 1} & 28 \\ 9 & 15 & 16 & 18 & 20 & 22 & \mathbf{2 2} & 39\end{array}$

Source: Staff projections and simulations.

1/ Variables include real GDP growth, growth of GDP deflator (in U.S. dollar terms), non-interest current account in percent of GDP, and non-debt creating flows.

2/ Assumes that the interest rate on new borrowing is by 2 percentage points higher than in the baseline., while grace and maturity periods are the same as in the baseline

3/ Exports values are assumed to remain permanently at the lower level, but the current account as a share of GDP is assumed to return to its baseline level after the shock (implicitly assuming an offsetting adjustment in import levels).

4/ Includes official and private transfers and FDI.

5/ Depreciation is defined as percentage decline in dollar/local currency rate, such that it never exceeds 100 percent.

6/ Applies to all stress scenarios except for A2 (less favorable financing) in which the terms on all new financing are as specified in footnote 2 . 
Figure 1. Ghana: Indicators of Public and Publicly Guaranteed External Debt under Alternatives Scenarios, 2009-2029 1/
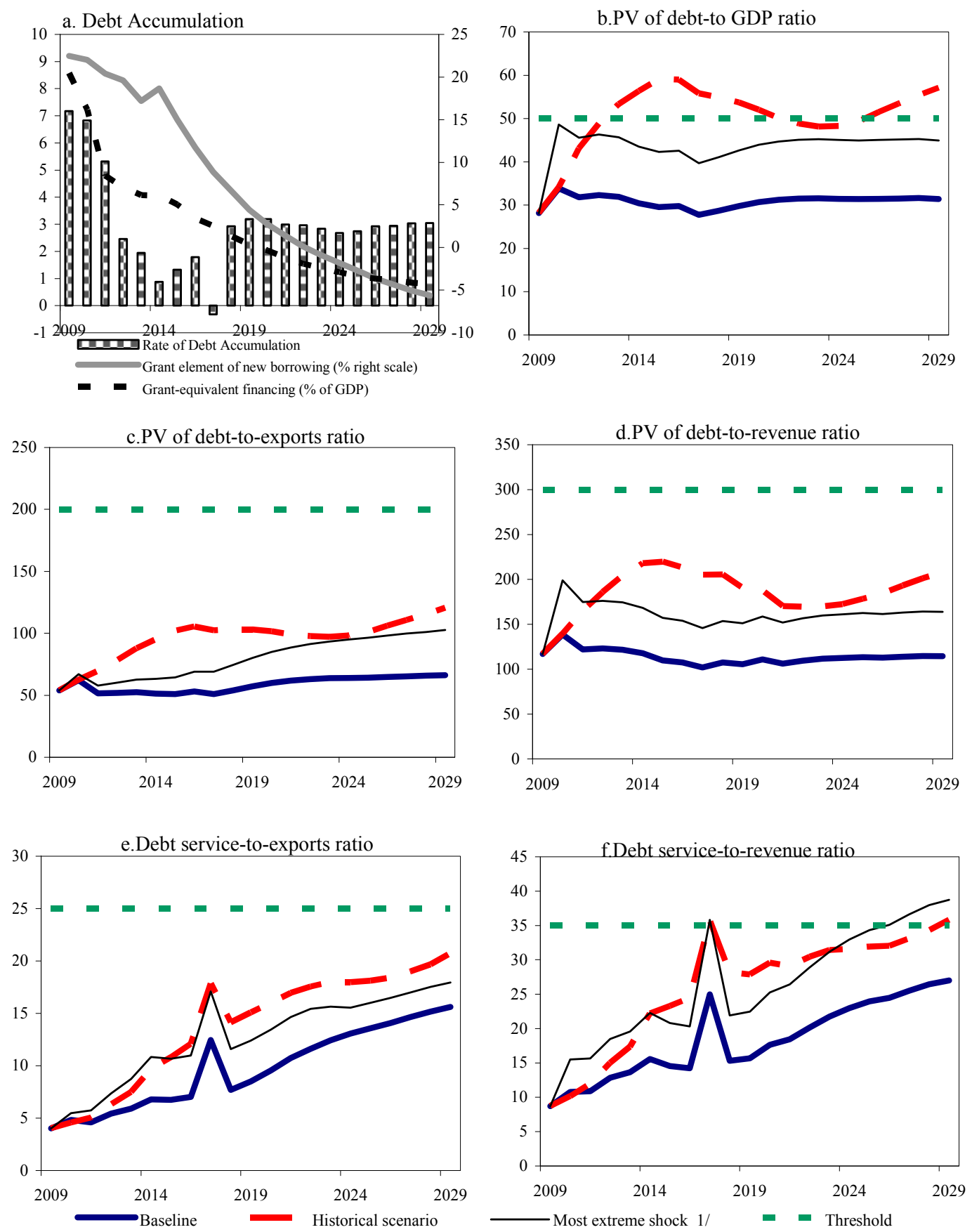

Source: Staff projections and simulations.

1 / The most extreme stress test is the test that yields the highest ratio in 2019. In figure b. it corresponds to a One-time depreciation shock; in c. to a Terms shock; in d. to a One-time depreciation shock; in e. to a Exports shock and in picture f. to a One-time depreciation shock 
Table 3. Ghana: Public Sector Debt Sustainability Framework, Baseline Scenario, 2006-2029

(In percent of GDP, unless otherwise indicated)

\begin{tabular}{|c|c|c|c|c|c|c|c|c|c|c|c|c|c|c|c|}
\hline & \multicolumn{3}{|c|}{ Actual } & \multirow[b]{2}{*}{ Average } & \multirow[b]{2}{*}{$\begin{array}{c}\text { Standard } \\
\text { Deviation }\end{array}$} & \multicolumn{5}{|l|}{ Estimate } & \multicolumn{5}{|c|}{ Projections } \\
\hline & 2006 & 2007 & 2008 & & & 2009 & 2010 & 2011 & 2012 & 2013 & 2014 & $\begin{array}{c}2009-14 \\
\text { Average }\end{array}$ & 2019 & 2029 & $\begin{array}{l}2015-29 \\
\text { Average } \\
\end{array}$ \\
\hline Public sector debt 1/ & 42.0 & 51.2 & 58.2 & 104.6 & 46.7 & 70.0 & 72.6 & 63.1 & 60.4 & 57.6 & 54.6 & 63.1 & 44.2 & 36.3 & 41.4 \\
\hline $\mathrm{o} / \mathrm{w}$ foreign-currency denominated & 17.2 & 24.8 & 29.2 & 79.7 & 46.4 & 41.5 & 47.7 & 44.1 & 43.9 & 43.3 & 41.4 & 43.7 & 38.5 & 33.5 & 37.2 \\
\hline Change in public sector debt & -36.3 & 9.1 & 7.0 & -4.7 & 27.7 & 11.8 & 2.6 & -9.5 & -2.6 & -2.8 & -3.1 & -0.6 & -1.3 & -1.5 & -1.2 \\
\hline Identified debt-creating flows & -5.8 & 0.8 & 4.1 & -0.5 & 29.2 & 4.7 & -1.3 & -12.1 & -3.0 & -2.9 & -2.8 & -2.9 & -2.2 & -2.2 & -1.9 \\
\hline Primary deficit & 4.2 & 6.1 & 10.7 & 3.6 & 3.8 & 5.3 & 2.4 & 0.7 & -0.2 & -0.1 & 0.0 & 1.4 & -0.4 & -0.4 & -0.2 \\
\hline Revenue and grants & 27.3 & 28.8 & 27.5 & 24.1 & 5.4 & 30.1 & 29.4 & 29.4 & 29.6 & 29.5 & 29.1 & 29.5 & 30.2 & 28.7 & 29.6 \\
\hline of which: grants & 5.4 & 6.1 & 4.7 & 4.4 & 2.2 & 6.0 & 5.0 & 3.3 & 3.3 & 3.3 & 3.3 & 4.0 & 2.0 & 1.3 & 1.8 \\
\hline Primary (noninterest) expenditure & 31.5 & 34.9 & 38.1 & 27.6 & 5.9 & 35.5 & 31.8 & 30.2 & 29.4 & 29.4 & 29.1 & 30.9 & 29.7 & 28.3 & 29.4 \\
\hline Automatic debt dynamics & -9.2 & -3.8 & -0.5 & -2.9 & 27.7 & 0.0 & -3.2 & -12.4 & -2.4 & -2.6 & -2.8 & -3.9 & -1.8 & -1.9 & -1.7 \\
\hline Contribution from interest rate/growth differential & -5.1 & -2.9 & -3.9 & -5.6 & 2.1 & -2.9 & -2.7 & -12.7 & -2.8 & -2.6 & -2.5 & -4.4 & -1.4 & -1.0 & -1.2 \\
\hline of which: contribution from average real interest rate & -0.4 & -0.7 & -0.5 & -0.4 & 1.2 & -0.4 & 0.6 & 1.4 & 1.4 & 0.4 & 0.3 & 0.6 & 0.4 & 1.0 & 0.6 \\
\hline of which: contribution from real GDP growth & -4.7 & -2.3 & -3.5 & -5.2 & 1.5 & -2.5 & -3.3 & -14.1 & -4.2 & -3.0 & -2.8 & -5.0 & -1.8 & -1.9 & -1.9 \\
\hline Contribution from real exchange rate depreciation & -4.1 & -0.9 & 3.4 & 2.7 & 26.7 & 2.9 & -0.4 & 0.4 & 0.3 & 0.0 & -0.3 & 0.5 & $\ldots$ & $\ldots$ & -0.2 \\
\hline Other identified debt-creating flows & -0.8 & -1.5 & -6.1 & -1.1 & 1.9 & -0.6 & -0.6 & -0.4 & -0.3 & -0.3 & 0.0 & -0.4 & 0.0 & 0.0 & 0.0 \\
\hline Privatization receipts (negative) & 0.0 & -0.8 & -5.7 & -0.7 & 1.8 & 0.0 & 0.0 & 0.0 & 0.0 & 0.0 & 0.0 & 0.0 & 0.0 & 0.0 & 0.0 \\
\hline Recognition of implicit or contingent liabilities & 0.0 & 0.0 & 0.0 & 0.0 & 0.0 & 0.0 & 0.0 & 0.0 & 0.0 & 0.0 & 0.0 & 0.0 & 0.0 & 0.0 & 0.0 \\
\hline Debt relief (HIPC and other) & -0.8 & -0.7 & -0.4 & -0.4 & 0.5 & -0.6 & -0.6 & -0.4 & -0.3 & -0.3 & 0.0 & -0.4 & 0.0 & 0.0 & 0.0 \\
\hline Other (specify, e.g. bank recapitalization) & 0.0 & 0.0 & 0.0 & 0.0 & 0.0 & 0.0 & 0.0 & 0.0 & 0.0 & 0.0 & 0.0 & 0.0 & 0.0 & 0.0 & 0.0 \\
\hline Residual, including asset changes & -30.5 & 8.3 & 3.0 & -4.3 & 13.6 & 7.1 & 4.0 & 2.5 & 0.4 & 0.1 & -0.3 & 2.3 & 0.8 & 0.7 & 0.7 \\
\hline \multicolumn{16}{|l|}{ Other Sustainability Indicators } \\
\hline PV of public sector debt & 24.8 & 26.4 & 50.0 & 26.9 & 9.2 & 58.9 & 60.2 & 51.8 & 49.2 & 46.7 & 44.0 & 51.8 & 35.9 & 34.6 & 35.2 \\
\hline $\mathrm{o} / \mathrm{w}$ foreign-currency denominated & 0.0 & 0.0 & 21.0 & 2.3 & 7.0 & 30.4 & 35.3 & 32.8 & 32.7 & 32.3 & 30.8 & 32.4 & 30.2 & 31.8 & 31.0 \\
\hline $\mathrm{o} / \mathrm{w}$ external & $\ldots$ & $\ldots$ & 21.0 & 21.0 & & 30.4 & 35.3 & 32.8 & 32.7 & 32.3 & 30.8 & 32.4 & 30.2 & 31.8 & 31.0 \\
\hline $\mathrm{PV}$ of contingent liabilities (not included in public sector debt) & $\ldots$ & $\ldots$ & $\ldots$ & & & $\ldots$ & $\ldots$ & & & & $\ldots$ & & $\ldots$ & $\ldots$ & \\
\hline Gross financing need $2 /$ & 16.5 & 16.8 & 22.5 & 19.0 & 4.0 & 18.0 & 15.4 & 11.6 & 10.2 & 9.1 & 9.9 & 12.4 & 11.2 & 8.3 & 10.3 \\
\hline $\mathrm{PV}$ of public sector debt-to-revenue and grants ratio (in percent) & 90.7 & 91.5 & 182.0 & 97.1 & 49.3 & 195.5 & 204.5 & 176.0 & 166.3 & 158.5 & 151.0 & 175.3 & 119.1 & 120.6 & 119.1 \\
\hline PV of public sector debt-to-revenue ratio (in percent) & 113.2 & 116.0 & 219.2 & 118.5 & 57.3 & 244.5 & 246.3 & 198.3 & 187.4 & 178.3 & 170.2 & 204.1 & 127.4 & 126.1 & 126.8 \\
\hline o/w external $3 /$ & & & 91.8 & 91.8 & & 126.3 & 144.4 & 125.6 & 124.4 & 123.4 & 119.2 & 127.2 & 107.1 & 116.0 & 111.6 \\
\hline Debt service-to-revenue and grants ratio (in percent) $4 /$ & 30.7 & 19.3 & 24.1 & 49.9 & 27.7 & 22.4 & 23.5 & 21.0 & 20.6 & 18.3 & 20.4 & 21.0 & 23.1 & 27.5 & 25.4 \\
\hline Debt service-to-revenue ratio (in percent) $4 /$ & 38.4 & 24.5 & 29.0 & 58.5 & 28.0 & 28.0 & 28.3 & 23.7 & 23.2 & 20.6 & 23.0 & 24.5 & 24.8 & 28.8 & 27.0 \\
\hline Primary deficit that stabilizes the debt-to-GDP ratio & 40.5 & -3.1 & 3.6 & 13.7 & 23.5 & -6.5 & -0.2 & 10.3 & 2.4 & 2.7 & 3.1 & 2.0 & 0.9 & 1.1 & 1.0 \\
\hline \multicolumn{16}{|l|}{ Key macroeconomic and fiscal assumptions } \\
\hline Real GDP growth (in percent) & 6.4 & 5.7 & 7.3 & 5.3 & 1.1 & 4.5 & 5.0 & 24.2 & 7.1 & 5.3 & 5.0 & 8.5 & 4.1 & 5.4 & 4.6 \\
\hline Average nominal interest rate on forex debt (in percent) & 1.4 & 2.1 & 3.8 & 1.9 & 0.9 & 4.3 & 3.1 & 3.2 & 3.0 & 2.9 & 2.9 & 3.2 & 2.9 & 5.0 & 3.6 \\
\hline Average real interest rate on domestic debt (in percent) & 3.4 & -2.4 & -3.4 & 0.7 & 4.9 & -4.3 & -0.9 & 3.4 & 5.1 & -0.1 & -0.8 & 0.4 & 0.6 & 0.3 & 0.9 \\
\hline Real exchange rate depreciation (in percent, + indicates depreciation) & -7.4 & -5.2 & 14.6 & -7.2 & 10.4 & 10.0 & & $\ldots$ & & & $\ldots$ & & $\ldots$ & $\ldots$ & $\ldots$ \\
\hline Inflation rate (GDP deflator, in percent) & 12.8 & 13.9 & 16.9 & 20.0 & 7.7 & 17.2 & 11.0 & 6.7 & 3.1 & 4.6 & 5.5 & 8.0 & 5.7 & 7.6 & 6.1 \\
\hline Growth of real primary spending (deflated by GDP deflator, in percent) & 0.2 & 0.2 & 0.2 & 0.1 & 0.1 & 0.0 & -0.1 & 0.2 & 0.0 & 0.1 & 0.0 & 0.0 & 0.0 & 0.0 & 0.0 \\
\hline Oil Fund assets (percent of GDP; end of period) & 0.0 & 0.0 & 0.0 & 0.0 & 0.0 & 0.0 & 0.0 & 0.0 & 0.0 & 3.9 & 6.8 & 11.6 & 13.4 & 0.0 & 6.4 \\
\hline Grant element of new external borrowing (in percent) & & & $\ldots$ & & 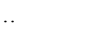 & 22.5 & 22.0 & 20.4 & 19.6 & 17.2 & 18.7 & 20.1 & 4.4 & -5.6 & \\
\hline
\end{tabular}

Sources: Country authorities; and Fund staff estimates and projections.

/ [Indicate coverage of public sector, e.g., general government or nonfinancial public sector. Also whether net or gross debt is used.]

2/ Gross financing need is defined as the primary deficit plus debt service plus the stock of short-term debt at the end of the last period

$3 /$ Revenues excluding grants.

4/ Debt service is defined as the sum of interest and anotization or medium and long-term deb.

$5 /$ Historical averages and standard deviations are generally derived over the past 10 years, subject to data availability. 


\begin{tabular}{|c|c|c|c|c|c|c|c|c|}
\hline & \multicolumn{8}{|c|}{ Projections } \\
\hline & 2009 & 2010 & 2011 & 2012 & 2013 & 2014 & 2019 & 2029 \\
\hline \multicolumn{9}{|c|}{ PV of Debt-to-GDP Ratio } \\
\hline Baseline & 59 & 60 & 52 & 49 & 47 & 44 & 36 & 35 \\
\hline \multicolumn{9}{|l|}{ A. Alternative scenarios } \\
\hline A1. Real GDP growth and primary balance are at historical averages & 59 & 61 & 65 & 66 & 66 & 66 & 73 & 90 \\
\hline A2. Primary balance is unchanged from 2009 & 59 & 63 & 58 & 61 & 63 & 65 & 85 & 113 \\
\hline A3. Permanently lower GDP growth $1 /$ & 59 & 60 & 52 & 50 & 48 & 45 & 42 & 50 \\
\hline \multicolumn{9}{|l|}{ B. Bound tests } \\
\hline B1. Real GDP growth at historic average minus one standard deviation in $2009-10$ & 59 & 61 & 52 & 50 & 48 & 45 & 40 & 41 \\
\hline B2. Primary balance is at historical average minus one standard deviations in $2009-10$ & 59 & 65 & 56 & 53 & 51 & 48 & 42 & 41 \\
\hline B3. Combination of B1-B2 using one half standard deviation shocks & 59 & 63 & 54 & 52 & 49 & 47 & 41 & 40 \\
\hline B4. One-time 30 percent real depreciation in 2010 & 59 & 74 & 63 & 60 & 57 & 55 & 51 & 61 \\
\hline B5. 10 percent of GDP increase in other debt-creating flows in 2010 & 59 & 70 & 60 & 57 & 54 & 51 & 45 & 43 \\
\hline \multicolumn{9}{|c|}{ PV of Debt-to-Revenue Ratio 2/ } \\
\hline Baseline & 196 & 204 & 176 & 166 & 158 & 151 & 119 & 121 \\
\hline \multicolumn{9}{|l|}{ A. Alternative scenarios } \\
\hline A1. Real GDP growth and primary balance are at historical averages & 196 & 208 & 215 & 218 & 220 & 222 & 241 & 313 \\
\hline A2. Primary balance is unchanged from 2009 & 196 & 214 & 199 & 206 & 214 & 224 & 281 & 392 \\
\hline A3. Permanently lower GDP growth $1 /$ & 196 & 205 & 177 & 168 & 161 & 156 & 140 & 174 \\
\hline \multicolumn{9}{|l|}{ B. Bound tests } \\
\hline B1. Real GDP growth at historic average minus one standard deviation in $2009-10$ & 196 & 206 & 178 & 169 & 162 & 155 & 134 & 143 \\
\hline B2. Primary balance is at historical average minus one standard deviations in $2009-10$ & 196 & 221 & 190 & 180 & 172 & 165 & 140 & 142 \\
\hline B3. Combination of B1-B2 using one half standard deviation shocks & 196 & 215 & 185 & 175 & 167 & 160 & 136 & 141 \\
\hline B4. One-time 30 percent real depreciation in 2010 & 196 & 251 & 214 & 202 & 194 & 187 & 170 & 211 \\
\hline B5. 10 percent of GDP increase in other debt-creating flows in 2010 & 196 & 237 & 203 & 192 & 184 & 176 & 150 & 148 \\
\hline \multicolumn{9}{|c|}{ Debt Service-to-Revenue Ratio 2/ } \\
\hline \multicolumn{9}{|l|}{ A. Alternative scenarios } \\
\hline A1. Real GDP growth and primary balance are at historical averages & 22 & 23 & 25 & 25 & 24 & 28 & 35 & 47 \\
\hline A2. Primary balance is unchanged from 2009 & 22 & 23 & 22 & 22 & 22 & 27 & 38 & 55 \\
\hline A3. Permanently lower GDP growth $1 /$ & 22 & 24 & 21 & 21 & 19 & 21 & 24 & 32 \\
\hline \multicolumn{9}{|l|}{ B. Bound tests } \\
\hline B1. Real GDP growth at historic average minus one standard deviation in 2009-10 & 22 & 24 & 21 & 21 & 19 & 21 & 24 & 29 \\
\hline B2. Primary balance is at historical average minus one standard deviations in $2009-10$ & 22 & 24 & 23 & 23 & 20 & 23 & 24 & 29 \\
\hline B3. Combination of B1-B2 using one half standard deviation shocks & 22 & 24 & 22 & 22 & 19 & 22 & 24 & 29 \\
\hline B4. One-time 30 percent real depreciation in 2010 & 22 & 26 & 26 & 27 & 25 & 29 & 35 & 53 \\
\hline B5. 10 percent of GDP increase in other debt-creating flows in 2010 & 22 & 23 & 23 & 24 & 22 & 24 & 25 & 30 \\
\hline
\end{tabular}

Sources: Country authorities; and Fund staff estimates and projections.

1/ Assumes that real GDP growth is at baseline minus one standard deviation divided by the square root of the length of the projection period.

2/ Revenues are defined inclusive of grants. 
Figure 2. Ghana: Indicators of Public Debt Under Alternative Scenarios, 2009-2029 1/

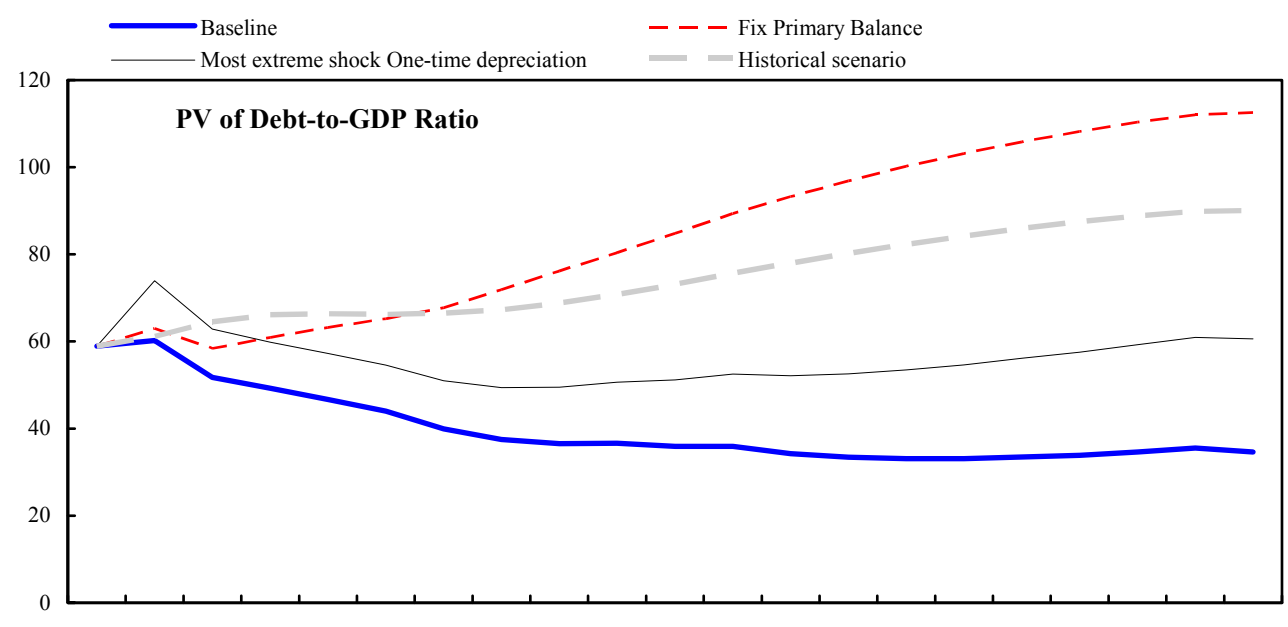

200920102011201220132014201520162017201820192020202120222023202420252026202720282029
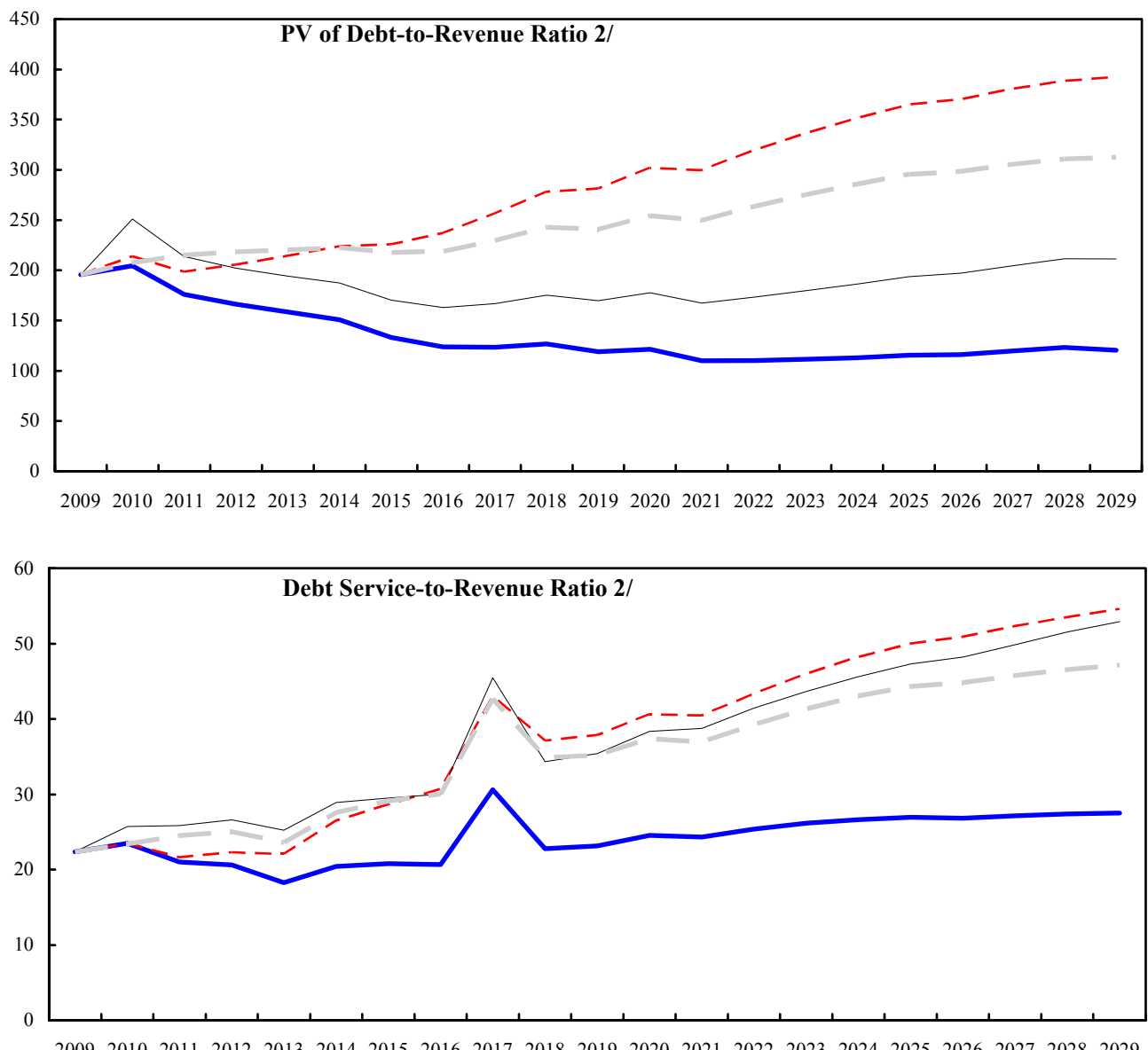

ources: Country authorities; and Fund staff estimates and projections.

$1 /$ The most extreme stress test is the test that yields the highest ratio in 2019 .

2 / Revenues are defined inclusive of grants. 


\section{INTERNATIONAL MONETARY FUND}

\section{GHANA}

2009 Article IV Consultation and Request for a Three-Year Arrangement Under the Poverty Reduction and Growth Facility

\section{Informational Annex}

Prepared by the African Department

June 29, 2009

\section{Contents}

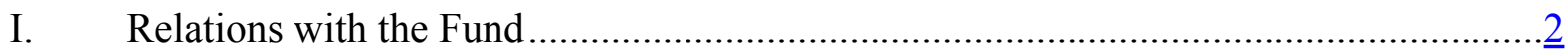

II. Joint World Bank-IMF Work Program, 2009-10 ......................................................

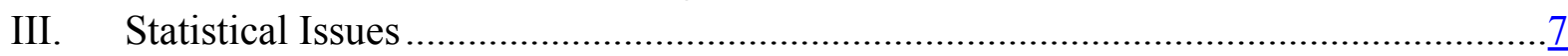




\section{RELATIONS WITH THE FUND}

(As of May 31, 2009)

I. Membership Status: Joined 09/20/1957;

$\underline{\text { Article VIII }}$

II. General Resources Account:

SDR Million

369.0

\% Quota

Quota

369.0

100.0

Fund holdings of currency

0.0

100.0

Reserve position in Fund

III. SDR Department:

SDR Million

\% Allocation

63.98

100.00

Holdings

0.05

0.09

IV. Outstanding Purchases and Loans:

PRGF Arrangements

SDR Million

105.45

\% Quota

28.58

V. Latest Financial Arrangements:

\begin{tabular}{|c|c|c|c|c|}
\hline Type & $\begin{array}{c}\text { Approval } \\
\text { Date }\end{array}$ & $\begin{array}{c}\text { Expiration } \\
\text { Date } \\
\end{array}$ & $\begin{array}{c}\text { Amount } \\
\text { Approved } \\
\text { (SDR Million) } \\
\end{array}$ & $\begin{array}{r}\text { Amount } \\
\text { Drawn } \\
\text { (SDR Million) } \\
\end{array}$ \\
\hline PRGF & $05 / 09 / 2003$ & $10 / 31 / 2006$ & 184.5 & 184.5 \\
\hline PRGF & 05/03/1999 & $11 / 30 / 2002$ & 228.8 & 176.2 \\
\hline PRGF & $06 / 30 / 1995$ & $05 / 02 / 1999$ & 164.4 & 137.0 \\
\hline
\end{tabular}

VI. Projected Payments to the Fund ${ }^{1}$

(SDR million; based on existing use of resources and present holdings of SDRs):

Principal

\begin{tabular}{rrrrr}
$\underline{6}$ & \multicolumn{5}{c}{ Forthcoming } \\
\cline { 3 - 5 } & $\underline{2010}$ & $\underline{2011}$ & $\underline{2012}$ & $\underline{2013}$ \\
$\underline{0.67}$ & $\underline{0.82}$ & $\underline{0.54}$ & 21.09 & 21.09 \\
0.67 & 0.82 & 11.34 & $\underline{0.72}$ & $\underline{0.62}$ \\
21.81 & 21.71
\end{tabular}

Charges/interest

Total

\footnotetext{
${ }^{1}$ When a member has overdue financial obligations outstanding for more than three months, the amount of such arrears will be shown in this section.
} 


\section{Implementation of HIPC Initiative:}

Enhanced Framework

A. Commitment of HIPC assistance

Decision point date

Feb 2002

Assistance committed by all creditors (US $\$$ million) ${ }^{2}$

$2,186.0$

Of which: IMF assistance (US\$ million)

112.1

(SDR equivalent in millions)

90.05

Completion point date

July 2004

B. Disbursement of IMF assistance (SDR million)

Assistance disbursed to the member $\quad 90.05$

Interim assistance $\quad 25.06$

Completion point balance $\quad 64.99$

Additional disbursement of interest income ${ }^{3} \quad 4.25$

$\begin{array}{ll}\text { Total disbursements } & 94.30\end{array}$

\section{Implementation of Multilateral Debt Relief Initiative (MDRI):}

A. MDRI-eligible debt (SDR million) ${ }^{4}$

Financed by MDRI Trust

Remaining HIPC resources

B. Debt relief by Facility (SDR million)

Delivery date

January 2006

\begin{tabular}{lcr}
\multicolumn{3}{c}{ Eligible Debt } \\
\hline$\underline{\text { GRA }}$ & $\underline{\text { PRGF }}$ & $\underline{\text { Total }}$
\end{tabular}

\section{Safeguards Assessment}

As Fund policy requires, the Bank of Ghana was subject to a safeguards assessment with respect to the PRGF arrangement approved on May 9, 2003. The assessment, which was completed on October 15, 2003, made recommendations for addressing specific

\footnotetext{
${ }^{2}$ Assistance committed under the original framework is expressed in net present value (NPV) terms at the completion point, and assistance committed under the enhanced framework is expressed in NPV terms at the decision point. Hence these two amounts ca not be added.

${ }^{3}$ Under the enhanced framework, an additional disbursement is made at the completion point corresponding to interest income earned on the amount committed at the decision point but not disbursed during the interim period.

${ }^{4}$ The MDRI provides 100 percent debt relief to eligible member countries that qualified for the assistance. Grant assistance from the MDRI Trust and HIPC resources provide debt relief to cover the full stock of debt owed to the Fund as of end-2004 that remains outstanding at the time the member qualifies for such debt relief.
} 
vulnerabilities in the external audit, financial reporting, internal audit, and internal controls areas. The authorities confirmed that all measures proposed were implemented. In accordance with IMF policy, an update safeguards assessment will be conducted no later than the completion of the first review under the proposed new three-year arrangement under the PRGF.

\section{Exchange Rate Arrangement}

On February 2, 1994, Ghana accepted obligations under Article VIII, Sections 2(a), 3, and 4, of the Fund's Articles of Agreement. The exchange rate regime is classified as a managed float with no pre-determined path. The system is free of restrictions on payments and transfers for current international transactions. At the end of May 2009, the average exchange rate for transactions in the interbank market was GHф1.475 per U.S. dollar.

\section{Article IV Consultation}

The 2008 Article IV consultation discussions were held in Accra during March 23-April 8, 2008. The staff report (Country Report No. 08/344) was discussed by the Executive Board on June 30, 2008 and is posted on the IMF website.

\section{FSAP Participation}

Ghana participated in the FSAP in 2000-01, and a Financial System Stability Assessment (FSSA) was issued to the Executive Board in 2001. An FSAP update was presented to the Board in December 2003.

\section{Technical Assistance}

$\underline{\text { Subject }}$

Advise on establishing large taxpayers unit

Review of public expenditure management

reforms

Tax policy

Fiscal ROSC

Regional advisor on public expenditure

management

Assessment of petroleum pricing mechanism

Public financial management

Enhancing fiscal discipline

Revenue administration

Tax policy

Revenue administration
Department

FAD

$2002 / 03$

FAD

2002/03

FAD

May 2003

FAD

Feb. 2004

FAD

2004/06

FAD

Jan. 2005

FAD

Mar.-Jun. 2006

FAD

May 2008

FAD

Jan. 2009

FAD

Apr. 2009

FAD

Apr. 2009 
Fiscal regime for natural resources

FAD

Jun. 2009

Accounting and internal audit reform

MFD

Jul./Nov. 2002

Mar. 2003

Foreign exchange market, government securities market, and banking system issues

MFD

Apr. 2003

Joint FSAP follow-up with World Bank

MFD

Jun. 2003

Multitopic technical assistance initiation

MFD

Nov. 2004

Improving monetary operations, banking

supervision and payment systems

MFD

Nov. 2004

Medium-term debt management strategy

$\mathrm{MCM}$

Mar. 2008

Money and banking statistics

STA

Jul. 2002

Jan.-Feb. 2004

Apr. 2007

Mar. 2008

Apr.-May 2009

National accounts statistics

STA

Sep./Oct. 2001

Aug.-Dec. 2002

Sep. 2003

Feb. 2009

National accounts and prices

STA

Mar. 2004

Oct. 2004

Apr.-May 2005

Apr.-May 2006

Sep. 2006

Government finance statistics

STA

Mar. 2005

May-Jun. 2006

May-Jun. 2009

Balance of payments statistic

STA

Feb. 2009

Pilot study of access to private capital markets ICM

May 2003

Nov. 2004

The remittance market

LEG

Apr.-May 2006

\section{Resident Representative}

The Fund has had a Resident Representative office in Accra since June 1985.

The current resident representative, Mr. M. Arnold McIntyre, assumed the post in August 2006. 
II. GHANA-JoInt WORLd BANK-IMF WORK Program, 2009-10

\begin{tabular}{|c|c|c|c|}
\hline Title & Products & $\begin{array}{l}\text { Provisional timing of } \\
\text { mission }\end{array}$ & $\begin{array}{l}\text { Expected delivery } \\
\text { date }\end{array}$ \\
\hline \multicolumn{4}{|c|}{ A. Mutual information on relevant work programs } \\
\hline $\begin{array}{l}\text { Bank work program } \\
\text { in next } 12 \text { months }\end{array}$ & $\begin{array}{l}\text { 1. Poverty } \\
\text { assessment } \\
\text { 2. Public expenditure } \\
\text { review } \\
\text { 3. PRSC } 7\end{array}$ & January 2010 & $\begin{array}{l}\text { November } 2009 \\
\text { June } 2010 \\
\text { April } 2010\end{array}$ \\
\hline $\begin{array}{l}\text { IMF work program } \\
\text { in next } 12 \text { months }\end{array}$ & $\begin{array}{l}\text { 1. First PRGF } \\
\text { review/2010 budget } \\
\text { discussions } \\
\text { 2. Second PRGF } \\
\text { review/2010 Article } \\
\text { IV consultation }\end{array}$ & $\begin{array}{l}\text { November } 2009 \\
\text { February } 2010\end{array}$ & $\begin{array}{l}\text { December } 2009 \\
\text { March } 2010\end{array}$ \\
\hline \multicolumn{4}{|c|}{ B. Requests for work program inputs } \\
\hline Fund request to Bank & $\begin{array}{l}\text { 1. Assessment of } \\
\text { progress with civil } \\
\text { service reform } \\
\text { 2. Assessment of } \\
\text { energy sector } \\
\text { recovery strategy }\end{array}$ & & $\begin{array}{l}\text { November } 2009 \\
\text { December } 2009\end{array}$ \\
\hline Bank request to IMF & $\begin{array}{l}\text { 1. Regular updates of } \\
\text { performance under } \\
\text { Fund-supported } \\
\text { program } \\
\text { 2. Regular updates of } \\
\text { macroeconomic } \\
\text { projections }\end{array}$ & & $\begin{array}{l}\text { Continuous } \\
\text { Continuous }\end{array}$ \\
\hline \multicolumn{4}{|c|}{ C. Agreement on joint products and missions } \\
\hline $\begin{array}{l}\text { Joint products in next } \\
12 \text { months }\end{array}$ & $\begin{array}{l}\text { 1. DSA } \\
\text { 2. FSAP }\end{array}$ & T.B.D. & $\begin{array}{l}\text { March } 2010 \\
\text { April } 2010\end{array}$ \\
\hline
\end{tabular}




\section{GHANA: STATISTICAL ISSUES}

1. Data provided to the Fund are broadly adequate for surveillance purposes, but the quality and timeliness of certain data need to be improved. There are notable deficiencies in the dissemination of statistical information to the public, as well as in the reporting for Fund publications, although the situation has improved recently with the publication on the Bank of Ghana's Web site of the Monetary Policy Committee Statement, Statistical Releases, and monthly monetary series for 2001-09. Data for publication in the International Financial Statistics (IFS) on international transactions were last reported for 2006, and monthly government finances for 2008, and on national accounts for 1997 . No quarterly balance of payments data are currently reported for publication in the IFS. The latest available data reported for publication in the Government Finance Statistics Yearbook (GFSY) are for 2008. However, these data cover only the cash revenue and expense transactions of the budgetary central government. Also, there have been long delays in the release by the Ghana Statistical Service (GSS) of census and survey results, irregularity in the quarterly statistical digest. The authorities are making efforts to improve the quality and timeliness of economic and financial data through participation in the Fund's General Data Dissemination System (GDDS) and the external sector and government finance statistics modules of the GDDS Project for Anglophone African Countries. GDDS metadata have been posted on the Fund's Dissemination Standards Bulletin Board (DSBB) since July 2005, but need to be updated.

\section{National accounts and prices}

2. The IMF's Statistics Department has provided TA on national accounts (NA) over the period 2001 to 2009. A "bundled" TA mission visited Accra during March 12-26, 2008, with a follow-up mission in February 2009. The main objectives of these missions were to assist the GSS in (a) developing a plan to adopt the System of National Accounts 1993 (1993 SNA); (b) rebasing the national accounts - currently dating from 1993; and (c) ensuring that plans are in place for a comprehensive data collection program to improve the source data.

3. The February 2009 mission confirmed the recommendations of the March 2008 TA mission that the 2004 Supply and Use Table (SUT) includes more updated and comprehensive sources. It confirmed that Ghana's national accounts are considerably understated, and recommended that the 2004, which consolidates new data sources, should be the basis for benchmarking the national accounts. The mission also pointed out some minor areas where changes to the estimates should be undertaken. There are still some concepts and methods that need to be addressed to insure compliance with the 1993 SNA. Incorporation of new data sources and rebasing of the national accounts to 2004, planned to be completed by end-2009. are likely to result in a significant upward revision of estimates of GDP. 
4. Improving the source data by creating a statistical system built on a regular survey program is a priority for increasing accuracy and reliability. In this regard the following areas require action: (i) design and building a business register that have a minimum of information on output values, if possible input values, and the number of employees. The 2003 National Industrial Census 2004/2 (NIC) serves as a good starting point, but must be maintained and extended to cover also service industries; (ii) create a national statistical system containing regular business surveys for manufacturing, construction, transport and communication, and other service industries (surveys on retail trade and NPISH are urgently needed); (iii) create a system for annual household surveys that is less comprehensive than the current Living Standards Survey (round 5) 2005/06 (GLSS) and which could be carried out on a more frequent basis; and (iv) carry out an agricultural census.

5. To address problems in the compilation of price statistics and national accounts, a peripatetic advisor was assigned to Ghana during 2001-04. Follow-up technical assistance has been provided under the GDDS project for Anglophone Africa. Work on updating the CPI weights using the fourth Ghana Living Standard Survey (1998/1999) was completed in mid-2006. In March 2007, STA assisted the GSS in developing a producer price index (PPI), which is now published.

\section{Labor statistics}

6. The scarcity of labor statistics is a cause for concern. Labor statistics are almost nonexistent, although some wage indicators are available from the Social Security National Insurance Trust (SSNIT). The Ministry of Employment has been receiving technical assistance from the United Nations Development Program and the International Labor Organization in the design and compilation of labor statistics.

7. Steps have been taken to improve fiscal data. The Controller Accountant General Department (CAGD) currently compiles monthly budget implementation reports, and the data are available within six weeks, although some factors undermine their reliability. There is a need for comprehensive and timely reconciliation of monthly treasury data with bank accounts. To address these shortcomings, the government has formed a committee to define the nature of "broad" and "narrow" government; moved to a system of immediate booking for "direct debits" and more frequent reporting of government account balances; and is implementing a new automated Budget and Public Expenditure Management System (BPEMS). The BPEMS covers ministries, departments, and agencies. However, the economic classification is not sufficiently detailed for data to be compiled in accordance with the requirements of GFSM 2001. In June 2006 a STA mission proposed classification of transactions in line with international guidelines.

8. There are also problems of transactional coverage. The CAGD and the BOG have been missing a substantial part of central government spending, such as donor flows disbursed directly to ministries and those arising from internally generated funds. They have also had difficulties in accounting for expenses paid by extrabudgetary funds. The operations 
of special funds, such as the SSNIT, the Ghana Education Trust Fund (GETF) and the District Assemblies Common Fund (DACF), are not yet covered in the fiscal accounts. Although the majority of local government expenses are directly met from budgetary accounts, the revenue of local governments and related spending, and transactions financed from the DACF are not yet covered. Extending the coverage of fiscal data to general government is strongly encouraged.

9. Comprehensive solutions to some of the data problems may have to await full implementation of the new BPEMS system and incorporation of Fund technical advice. Various missions from FAD have suggested short-term, temporary solutions to alleviate current data quality problems. A joint Bank-Fund mission in 2004 assessed progress on monitoring poverty-related spending through the Heavily Indebted Poor Countries (HIPC) Assessments and Actions Plans; a Fiscal Transparency Report on Observance of Standards and Codes (ROSC) was undertaken in July 2004; and STA provided further technical assistance in March 2005.

\section{The country has elected to participate in the UK Department for International} Development (DFID) under the Phase 2 initiative, which is a continuation of the DFID General Data Dissemination System Project for Anglophone Africa. The purpose of this project is to enhance the capacity of participating countries' statistical systems. The DFID Phase 2 Project will rely on the GDDS framework to introduce improvements in fiscal reporting based on the methodology of the GFSM 2001. The Project ends in April 2009 and is expected that two or three missions will be required to complete the GFS module. An STA mission recommended an enhancement of the chart of accounts so as to include financing transactions and additional details on revenue and expense classifications. This mission also assisted the authorities with bridging their data with the GFSM 2001 framework. It recommended establishing an interagency working group to delineate the general government sector to ensure consistency of coverage amongst all datasets. The mission has also initiated the compilation of data for extrabudgetary and social security funds and recommended, as a first step, to expand coverage of fiscal data to at least the consolidated central government.

\section{Monetary statistics and international reserves}

11. While BOG had made significant progress implementing the previous missions' recommendations on monetary and financial statistics, continued efforts are needed to improve the methodological soundness of the data. There have been three mission on to Ghana during 2007-09 in this area. The $\mathbf{2 0 0 7}$ mission focused on the residency criterion in the BOG accounting data used as the source to generate monetary statistics, and in the sectorization of the government accounts with other depository corporations (ODCs). The mission assisted the BOG to automatically derive the standard forms to report monetary statistics (SRFs) for the central bank (SRF 1SR) and the ODCs (SRF 2SR) to STA, in light of the recommendations of the Monetary and Financial Statistics Manual. The BOG currently reports monthly data with a lag of four to six weeks, and data from the ODCs with a lag of 
eight to ten weeks. The March 2008 mission found that significant progress has been achieved in implementing STA's earlier recommendations, especially to improve data for the ODCs. The mission agreed with the BoG staff on a plan to expand the coverage of the ODCs to include the savings and loans companies, rural banks, money market mutual funds, and credit unions; and compiled a comprehensive list of the financial corporations operating in Ghana, including the timing and frequency of the data reported to the various regulatory agencies. The mission proposed that the BoG starts compiling the Other Financial Corporations (OFCs) Survey, covering finance and leasing companies.

\section{The April-May 2009 mission concluded that the implementation of earlier advice} was uneven. While substantial progress was made in strengthening the source data for the BOG and expanding institutional coverage of the ODCs to include rural banks and savings and loans companies, the progress in improving data collection and compilation from the OFCs had been slow. To enhance coordination among financial regulatory agencies and improve data collection on the OFCs, the authorities agreed to establish an inter-agency committee. The mission helped BOG migrate to standardized report forms and an integrated monetary database, as well as expand the institutional coverage (see above). With the wider coverage of institutions, the authorities need to reconsider the definition of broad money, which currently only includes DMBs and thus may understate the aggregate.

\section{Debt statistics}

\section{The responsibility for external debt recording and payment is divided among} three agencies. The MOFEP, through its Aid and Debt Management Unit (ADMU), maintains the external debt database. It is responsible for recording debt-payment obligations, issuing payment requests, and tracking HIPC debt relief. The CAGD confirms the legality of the payment and authorizes the release of public funds. It is responsible for accounting for debt payments and rendering reports to parliament. The BOG as the payment agent for the government verifies payments made to ADMU and CAGD.

\section{An FAD technical assistance mission in 2001 concluded that the three institutions} needed to improve the transparency and accountability of external debt management. The authorities should (i) develop a single computerized database that is available to all three institutions; (ii) formalize procedures used for settling debt payments (including obtaining debt notification from donors, delegating signing authorities of officials within the relevant organizations, and creating registers tracing the movement of the documents required to effect external debt payment); and (iii) improve the analytical content and timeliness of data, which are not currently reported at regular intervals.

\section{To enable systematic comparison of the budget, the balance of payments, and the} BOG cash-flow data, the authorities should clearly identify the government subsectors for which data are reported and prepare a clear classification of financing, outstanding debt, and guarantees issued. 


\section{Trade and balance of payments statistics}

16. Since 1982 the BOG Research Department has had primary responsibility for the compilation and presentation of the annual and quarterly balance of payments (BOP) statistics. Data are compiled based on the Balance of Payments Manual, Fifth Edition (BPM5). The main data sources were the Customs Excise and Preventive Service (CEPS), administrative data (government ministries and departments within BOG), commercial banks, and the GSS. In addition, the BOG carried out simple financial surveys on other corporate entities that are involved in transactions with nonresidents. Starting from 2007, Ghana participated in the external sector module of the DFID project. Three TA missions have been conducted under the auspices of this project to undertake a comprehensive enterprise survey of cross-border financial flows and stocks with a view to significantly improving the quality of BOP and international investment position (IIP). The latest February/March 2009 TA mission finalized survey data entry, and verified and grossed-up survey results to construct BOP and IIP statements. The mission concluded that the survey findings are of good quality and should be incorporated into BOP compilation program. The BOG should contribute towards further success of other surveys by pursuing activities that promote improvement of standards and timeliness of reporting of financial statements by enterprises. Efforts in this direction could be focused on non-multinational enterprises whose financial statements were in many cases not comprehensive. Partnerships with accounting bodies and other institutions such as the auditor general's office will be explored in this direction.

\section{Currently, the GSS is not publishing timely monthly trade statistics, although} the data are available from the CEPS. The staff has recommended that the GSS collaborate with the CEPS to process customs data within six weeks and with the Ministry of Trade and Industry (MOT) and the BOG to identify and reduce discrepancies in trade statistics and to ensure that imports into bonded warehouses are not double-counted. Data collection procedures of the CEPS need to be improved, and there is also room for improving trade volume data collected by the CEPS through customs invoices, which would help the GSS to extract meaningful import and export unit values.

\section{Fund staff has recommended that the GSS produce export unit values for major} export commodities, such as gold and cocoa. A high coverage of the country's export bundle can be obtained from just three major exports - cocoa, gold, and unwrought aluminum. In contrast, deflation of imports is likely to require an iterative procedure to strike a balance between coverage of the index and its stability, owing to the heterogeneity of the basket. 


\section{GHANA: TABLE OF COMMON INDICATORS REQUIRED FOR SURVEILLANCE} (AS OF MAY 31, 2009)

\begin{tabular}{|c|c|c|c|c|c|}
\hline & $\begin{array}{c}\text { Date of latest } \\
\text { observation }\end{array}$ & $\begin{array}{l}\text { Date } \\
\text { received }\end{array}$ & $\begin{array}{c}\text { Frequency } \\
\text { of } \\
\text { Data }^{6}\end{array}$ & $\begin{array}{l}\text { Frequency } \\
\quad \text { of } \\
\text { Reporting }^{6}\end{array}$ & $\begin{array}{l}\text { Frequency } \\
\quad \text { of } \\
\text { Publication }^{6}\end{array}$ \\
\hline Exchange Rates & May, 2009 & May 2009 & $\mathrm{D}$ & W & $\mathrm{D}$ \\
\hline $\begin{array}{l}\text { International Reserve Assets and Reserve } \\
\text { Liabilities of the Monetary Authorities }{ }^{1}\end{array}$ & May 2009 & June 2009 & M & M & Q \\
\hline Reserve/Base Money & May 2009 & June 2009 & $\mathrm{~W}$ & M & I \\
\hline Broad Money & April 2009 & June 2009 & M & M & I \\
\hline Central Bank Balance Sheet & May 2009 & June 2009 & M & M & I \\
\hline $\begin{array}{l}\text { Consolidated Balance Sheet of the Banking } \\
\text { System }\end{array}$ & April 2009 & June 2009 & M & M & I \\
\hline Interest Rates ${ }^{2}$ & May 2009 & June 2009 & M & M & M \\
\hline Consumer Price Index & April 2009 & May 2009 & M & M & M \\
\hline $\begin{array}{l}\text { Revenue, Expenditure, Balance and } \\
\text { Composition of Financing }{ }^{3}-\text { general } \\
\text { government }\end{array}$ & NA & NA & NA & NA & NA \\
\hline $\begin{array}{l}\text { Revenue, Expenditure, Balance and } \\
\text { Composition of Financing }{ }^{3}-\text { central } \\
\text { government }\end{array}$ & March 2009 & May 2009 & M & M & I \\
\hline $\begin{array}{l}\text { Stocks of Central Government and Central } \\
\text { Government-Guaranteed Debt }\end{array}$ & Dec. 2008 & May 2009 & M & Q & I \\
\hline External Current Account Balance & March 2009 & May 2009 & Q & Q & Q \\
\hline Exports and Imports of Goods and Services & March 2009 & May 2009 & Q & Q & I \\
\hline GDP/GNP & 2008 & May 2009 & A & A & I \\
\hline Gross External Debt & Dec. 2008 & May 2009 & M & I & A \\
\hline International Investment Position $^{7}$ & NA & NA & NA & NA & NA \\
\hline
\end{tabular}

${ }^{1}$ Includes reserve assets pledged or otherwise encumbered as well as net derivative positions.

${ }^{2}$ Both market-based and officially determined, including discount rates, money market rates, rates on treasury bills, notes and bonds.

${ }^{3}$ Foreign, domestic bank, and domestic nonbank financing.

${ }^{4}$ The general government consists of the central government (budgetary funds, extrabudgetary funds, and social security funds) and state and local governments.

${ }^{5}$ Including currency and maturity composition.

${ }^{6}$ Daily (D); Weekly (W); Monthly (M); Quarterly (Q); Annually (A); Irregular (I); Not Available (NA)

${ }^{7}$ Includes external gross financial assets and liability positions vis-à-vis non residents. 


\section{Statement by the Staff Representative on Ghana}

July 15, 2009

This statement provides information on recent economic developments in Ghana that has become available since the staff report was circulated to the Board on June 30, 2009. These developments do not change the thrust of the staff appraisal.

Preliminary fiscal data for January to April 2009. These show a fiscal deficit of GHc 359 million, slightly less than half of the January-June indicative ceiling. The fiscal data have been posted on the Ministry of Finance website, consistent with the government's goals to strengthen fiscal transparency (MEFP, $₫ 69$ ).

Public sector wage negotiations. Agreement has been reached with the education service on a 17 percent wage increase for 2009, retroactive to January. This is consistent with the budget's wage bill provision, and is expected to establish a benchmark for wage setting with other public workers.

Projects being considered for non-concessional foreign financing. A listing has been compiled and provided to staff, advancing the earlier timetable to make this submission at the time of the first PRGF review (MEFP, $\mid 54$ ). The projects are mostly related to energy and transportation infrastructure, and the government will review these projects with staff, as outlined in the MEFP, at the time of the first review. Pending this discussion, the government has reiterated its intention not to borrow on nonconcessional terms in foreign currency, except for possible guarantees for GNPC oil and gas-related borrowing described in the MEFP.

Consumer price inflation. 12-month CPI inflation rose from 20.1 percent in May to 20.7 percent in June. Excluding the impact of a 30 percent rise in petroleum product prices in early-June, inflation would have fallen to 20.0 percent in June, slightly above the indicative inflation target of 19.7 percent in the government's program (MEFP, table 1).

Publication authorization. The authorities have communicated their consent for the Fund to publish the staff report for the 2009 Article IV Consultation discussions and request for a three-year arrangement under the PRGF and all related documents. 


\section{INTERNATIONAL MONETARY FUND}

EXTERNAL

Public Information Notice

Public Information Notice (PIN) No. 09/86

FOR IMMEDIATE RELEASE

July 17,2009
International Monetary Fund

$70019^{\text {th }}$ Street, NW

Washington, D. C. 20431 USA

\section{IMF Executive Board Concludes Article IV Consultation with Ghana}

On July 15, 2009, the Executive Board of the International Monetary Fund (IMF) concluded the Article IV consultation with Ghana. ${ }^{1}$

\section{Background}

Ghana's economic growth rose to a two-decade high of 7.3 percent in 2008 . This reflected expansionary fiscal policies combined with an upswing in private sector activity based on strong credit expansion, buoyant remittances, and strong agricultural yields.

Inflation rose on account of external shocks and strong domestic demand. After falling briefly to single-digits in 2006, inflation rose through 2007-08, reflecting global food and fuel price shocks, strong domestic demand, and the pass through from currency depreciation. In January-May 2009, inflation stabilized in the 20 percent range.

The fiscal deficit surged to 14.5 percent of GDP in 2008 , reflecting rapid public spending growth, with capital spending, energy subsidies, and wage and salaries each rising by more than 1 percentage point of GDP. The 2008 fiscal deficit drew on exceptional foreign financing amounting to 7.3 percent of GDP, comprising resources from the late-

\footnotetext{
${ }^{1}$ Under Article IV of the IMF's Articles of Agreement, the IMF holds bilateral discussions with members, usually every year. A staff team visits the country, collects economic and financial information, and discusses with officials the country's economic developments and policies. On return to headquarters, the staff prepares a report, which forms the basis for discussion by the Executive Board. At the conclusion of the discussion, the Managing Director, as Chairman of the Board, summarizes the views of Executive Directors, and this summary is transmitted to the country's authorities.
} 
2007 Eurobond issue and proceeds from the sale of Ghana Telecom. In addition, the central bank provided financing equivalent to more than 3 percent of GDP.

Monetary policy was tightened in response to rising inflation. The Bank of Ghana increased its benchmark lending rate by 6 percentage points between October 2007 and February 2009, and market conditions tightened by more, as reflected in interbank and treasury bill rates. The policy rate was left unchanged in May 2009 on an assessment that inflationary pressures were easing. Notwithstanding interest rate increases, the prime rate has been lower than annual inflation since early-2008, while real interbank rates have been only slightly positive. The banking sector has continued to register strong asset growth, with private sector credit up 56 percent in the year to April 2009. This expansion continues to reflect local deposit mobilization, with broad money up 28 percent on the same basis.

Ghana's overall financial system remains stable. The regulatory and supervisory framework is strong, backed by a modern payment and settlement infrastructure. Financial Soundness Indicators (FSIs) point to a banking system that is liquid and with capital above statutory levels. However, some recent deterioration is evident: nonperforming assets are high and rising (9.6 percent in March 2009); profitability is declining; and a few banks are financially strained. The Bank of Ghana has required banks to raise their minimum capital to $\mathrm{GH} \phi 60$ million (about $\$ 40$ million) to allow them to play a larger role in the rapidly growing economy. The non-banking sector (insurance, pensions and capital markets) has continued to perform well.

Ghana's external current account deficit widened to 19 percent of GDP in 2008, up from 12 percent of GDP a year earlier. This largely reflected a 33 percent increase in non-oil imports values, driven by strong domestic demand. Financing was provided through an increased external capital account surplus, buoyed by Ghana Telecom privatization proceeds $\left(5 \frac{1}{2}\right.$ percent of GDP), and a draw-down of gross international reserves from $\$ 2.8$ billion to $\$ 2.0$ billion. Reflecting the latter, gross reserve cover declined from 2.7 to 2.2 months of projected import cover. In the first quarter of 2009, exports have remained buoyant, but private remittances fell by 18 percent from a year earlier.

Exchange rate flexibility has increased since mid-2008. Reflecting Ghana's macroeconomic imbalances, the exchange rate depreciated about 50 percent against the dollar during 2008 and the first half of 2009. Much of this adjustment was offset, however, by Ghana's high inflation rate and the appreciation of the dollar in the context of the global financial crisis. Accordingly, the real effective exchange rate in April 2009 was just 8 percent more depreciated than in 2007. Over the recent period, illiquidity in the foreign currency market has been associated with a decline in interbank trading and a widening of spreads. 


\section{Executive Board Assessment}

Executive Directors noted that after a decade of gains in real income and poverty reduction, Ghana's macroeconomic situation had weakened owing to exogenous shocks and highly expansionary fiscal policies, in particular in the run-up to the elections, that contributed to higher inflation and large external imbalances. While Ghana's terms of trade are projected to strengthen in 2009, supporting incomes and growth, Directors recognized the uncertain environment and the downside risks. Starting in 2011, oil production is expected to bolster growth and fiscal revenues, creating new challenges for macroeconomic management.

Directors supported the authorities' efforts to restore macroeconomic stability by seeking to achieve fiscal sustainability. They endorsed as appropriately ambitious the deficit target of 9.4 percent of GDP for 2009, welcoming the combination of expenditure restraint and revenue enhancing measures. Noting that Ghana remains at moderate risk of debt distress, Directors emphasized that further fiscal consolidation will be critical over the medium-term. A number of Directors stressed the importance of pursuing prudent debt management, especially as regards nonconcessional borrowing. Reforming mandatory and inflexible budgetary outlays would create space for more productive and priority development spending. Directors also supported the maintenance of Ghana's broad social safety net program that should protect vulnerable groups. They were encouraged that the authorities stand ready to take additional measures to achieve their deficit targets.

Directors observed that expected oil revenues would create new fiscal space but on a relatively modest scale and for a fairly short period. They emphasized, therefore, that the authorities should not be complacent about the fiscal outlook and new revenues should be used wisely. The authorities' intention to extend Ghana's participation in the Extractive Industries Transparency Initiative to the oil sector was welcomed. Directors believed that high priority should be given to strengthening public financial management, enhancing the efficiency of public spending, and strengthening domestic revenue mobilization.

Directors noted that inflation in 2008 had substantially exceeded the authorities' target, largely due to highly expansionary fiscal policies which were not fully offset by monetary tightening. With a tighter fiscal stance, it would be an opportune time for monetary policy to reduce inflation. Therefore, in light of the negative real interest rates and the need to increase credibility of the inflation targeting framework, Directors supported a further tightening in the monetary stance. They concurred with the authorities' renewed emphasis on communications and transparency to enhance the inflation-targeting framework. 
Directors welcomed the authorities' intention to maintain a flexible exchange rate regime consistent with their inflation-targeting framework. They also welcomed the authorities' plans to use a possible SDR allocation to rebuild foreign exchange reserves. Directors noted the staff's assessment that the recent depreciation had partly corrected the earlier overvaluation.

Directors observed that Ghana's financial system has so far been generally insulated from the global crisis. They recognized, however, that strains had emerged after several years of rapid banking expansion, including a deterioration in loan portfolios. While noting progress made in supervisory capacity, Directors pointed to the need to further strengthen supervision, including cross-border coordination, and to reinforce risk management and corporate governance. Directors welcomed the authorities' request for a Financial Sector Assessment Program update.

Directors encouraged the authorities to enhance the quality and timeliness of data reporting to the Fund. They welcomed the authorities' plans to improve estimates of national accounts statistics following recent IMF technical assistance.

Public Information Notices (PINs) form part of the IMF's efforts to promote transparency of the IMF's views and analysis of economic developments and policies. With the consent of the country (or countries) concerned, PINs are issued after Executive Board discussions of Article IV consultations with member countries, of its surveillance of developments at the regional level, of post-program monitoring, and of ex post assessments of member countries with longer-term program engagements. PINs are also issued after Executive Board discussions of general policy matters, unless otherwise decided by the Executive Board in a particular case. 
Ghana: Selected Economic Indicators, 2005-09

\begin{tabular}{|c|c|c|c|c|c|}
\hline & $\begin{array}{l}2005 \\
\text { Act. }\end{array}$ & $\begin{array}{l}2006 \\
\text { Act. }\end{array}$ & $\begin{array}{c}2007 \\
\text { Act }\end{array}$ & $\begin{array}{l}2008 \\
\text { Act. }\end{array}$ & $\begin{array}{l}2009 \\
\text { Proj. }\end{array}$ \\
\hline Production and prices & \multicolumn{5}{|c|}{ (Annual change in percent) } \\
\hline Real GDP & 5.9 & 6.4 & 5.7 & 7.3 & 4.5 \\
\hline CPI inflation (end of period) & 14.8 & 10.9 & 12.7 & 18.1 & 14.6 \\
\hline Central government & \multicolumn{5}{|c|}{ (Percent of GDP } \\
\hline Total revenue and grants & 27.1 & 27.3 & 28.8 & 27.5 & 30.0 \\
\hline Of which: grants & 5.2 & 5.4 & 6.1 & 4.7 & 6.0 \\
\hline Expenditure and net lending & 30.7 & 34.4 & 37.3 & 41.0 & 36.7 \\
\hline Of which: capital expenditure & 12.0 & 12.4 & 14.4 & 15.7 & 12.6 \\
\hline Overall balance & -4.9 & -7.5 & -9.2 & -14.5 & -9.4 \\
\hline Gross government debt & 78.3 & 42.0 & 51.2 & 58.2 & 66.4 \\
\hline Monetary sector & \multicolumn{5}{|c|}{ (Annual change in percent) } \\
\hline Broad money & 13.7 & 38.8 & 35.9 & 40.2 & 21.7 \\
\hline Velocity of broad money & 4.3 & 3.5 & 3.0 & 2.8 & 3.0 \\
\hline Real private sector credit & 28.7 & 28.7 & 41.6 & 25.5 & 9.0 \\
\hline External sector & \multicolumn{5}{|c|}{$\begin{array}{c}\text { (Millions of U.S. dollars, unless otherwise } \\
\text { indicated) }\end{array}$} \\
\hline Exports of goods and services & 3,910 & 5,111 & 6,004 & 7,071 & 7,509 \\
\hline Imports of goods and services & 6,620 & 8,287 & 10,065 & 12,569 & 11,285 \\
\hline Current account balance & -885 & $-1,265$ & $-1,805$ & $-3,110$ & $-1,869$ \\
\hline Percent of GDP & -8.3 & -9.9 & -12.0 & -19.3 & -13.0 \\
\hline Gross official reserves & 1,895 & 2,270 & 2,837 & 2,036 & 1,950 \\
\hline $\begin{array}{l}\text { Months of imports of goods and } \\
\text { services }\end{array}$ & 2.7 & 2.7 & 2.7 & 2.2 & 1.9 \\
\hline
\end{tabular}


July 16, 2009

\section{IMF Executive Board Approves US\$602.6 Million PRGF Arrangement for Ghana}

The Executive Board of the International Monetary Fund (IMF) has approved a threeyear arrangement under the Poverty Reduction and Growth Facility (PRGF) for Ghana in an amount equivalent to SDR 387.45 million (about US $\$ 602.6$ million) to support the government's economic program to tackle macroeconomic instability. The approval will enable an initial disbursement of SDR 67.65 million (about US\$105.2 million) immediately.

At the conclusion of the Executive Board's discussion on Ghana's request for a PRGF arrangement, which was held on July 15, 2009, Mr. Takatoshi Kato, Deputy Managing Director and Acting Chair, stated:

"Ghana's macroeconomic conditions deteriorated substantially during 2008, reflecting global shocks to food and fuel prices and highly expansionary fiscal policies, in particular in the run-up to the elections. Inflation rose to about 20 percent and the current account deficit widened appreciably, putting pressure on Ghana's international reserves and the exchange rate. Growth is projected to moderate in 2009, with potential additional downside risks stemming from the global recession.

“The authorities' economic adjustment program appropriately centers on efforts to reestablish macroeconomic stability. The budget deficit target of 9.4 percent of GDP in 2009 , down from 14.5 percent in 2008 , is appropriately ambitious. In light of the limited scope for countercyclical fiscal policy in Ghana, the authorities stand ready to take additional measures to achieve their deficit targets in the event of revenue shortfalls.

"The authorities' planned reduction of the budget deficit to $4 \frac{1}{2}$ percent of GDP in 2011 , and below this level in subsequent years, will be critical to restoring public debt sustainability. To help achieve this fiscal goal, the authorities should ensure that 
petroleum pricing and utility tariffs allow for full cost recovery to avoid large subsidy costs. More effective control will also be needed over the public sector wage bill, and steps should be taken to modernize the tax regime and strengthen collection.

"Oil revenues that are expected to start in 2011 will create important new fiscal space and potentially bring Ghana close to middle-income status. While these revenues can help to support Ghana's fiscal consolidation, the authorities should not be complacent about the fiscal outlook in 2011 and beyond. The horizon for oil production could prove relatively short, and it will be important that the new revenues be used wisely. Accordingly, high priority should be given to strengthening public financial management under the authorities' program.

"Monetary policy implementation under the authorities' inflation targeting framework aims to reduce inflation to single-digit levels by 2010 . While the disinflation process will be supported by the planned fiscal consolidation, rising global oil prices and continuing depreciation of the Ghanaian cedi pose risks to inflation. The Bank of Ghana should be ready to tighten monetary conditions further should conditions warrant. Looking forward, the Bank of Ghana should also strengthen its communications strategy and the transparency of the inflation targeting framework.

"Ghana's financial system has so far been relatively resilient in the face of global developments, but vulnerabilities have emerged following rapid banking sector expansion in recent years, and loan portfolios have deteriorated. The authorities should ensure close supervision and encourage commercial banks to reinforce risk management and corporate governance practices. Gaps in cross border supervision will also require stronger regional collaboration.

"The depreciation of the cedi since the second half of 2008 has helped the process of balance of payments adjustment, and continued flexibility will be important. Efforts should continue to rebuild foreign exchange reserves in order to enhance Ghana's ability to weather future external shocks. 
ANNEX

\section{Recent Economic Developments}

Ghana's growth remained strong in 2008 at 7.3 percent, up from 5.7 percent of GDP in 2007. Since 2000 , growth has been supported by significant debt relief, which provided the country with fiscal space to invest in infrastructure, and the social sectors. Thanks to the combination of higher growth, declining inflation and improved social spending, poverty levels have significantly declined. Ghana is poised to achieve the Millennium Development Goal of halving extreme poverty ahead of 2015.

Fiscal performance deteriorated sharply during 2007-08, partly due to a severe energy crisis in 2006-2007 and the global food and fuel crisis in 2008, but also importantly due to highly expansionary fiscal policies ahead of the end-2008 presidential elections. As a consequence, the fiscal deficit jumped from 9.2 percent of GDP in 2007 to 14.5 percent in 2008.

The strong public spending combined with rapid credit expansion contributed to a large increase in inflation and a deterioration of the external current account. Year-on-year inflation rose to the 20 percent range in early-2009 from 12.7 percent in 2007 , while the current account deficit widened to 19.3 percent of GDP in 2008, compared with a 12.0 percent of GDP in 2007 . The overall balance of payment recorded a deficit of US $\$ 941$ million in 2008 , compared with a surplus of US $\$ 413$ million in 2007 , with the former financed by a drawdown of international reserves.

\section{Program Summary}

The government's medium-term macroeconomic program builds on Ghana's second Poverty Reduction Strategy Paper. It aims to substantially reduce Ghana's large fiscal imbalances by 2011 and put in place strengthened institutions for public financial management.

The macroeconomic framework for 2009-11 aims to achieve:

- A real non-oil GDP growth of about $5 \frac{1}{2}$ percent on average.

- A medium-term inflation to 7-9 percent per year.

- An overall budget deficit of 4.5 percent of GDP in 2011.

- International reserves coverage equal to about 3 months of imports.

To achieve these objectives, medium-term policies include: 
- Reducing the deficit, including through revenue mobilization, cuts in low-priority spending, and flexible pricing of energy products to avoid costly subsidies.

- Implementing a comprehensive program to improve public financial management.

- Implementing a comprehensive public sector reform program.

-Further strengthening the recently-adopted inflation targeting regime, including by a revamped central bank communications strategy 


\section{Statement by Jafar Mojarrad, Executive Director for Ghana and John Kwabena Kwakye, Advisor to Executive Director July 15, 2009}

My Ghanaian authorities appreciate the constructive dialogue with staff on the Article IV consultation and the PRGF arrangement. After a decade of strong gains in per capita incomes and poverty reduction, Ghana's macroeconomic performance has weakened in the past three years due to adverse exogenous factors, including the fuel and food price shocks and the spillovers of the global financial crisis and economic slowdown, but also following increasingly expansionary policies, especially in the run up to the presidential and parliamentary elections. Having assumed the reins of power only in January 2009 after the elections, the new government's overriding objective is to achieve economic prosperity for all Ghanaians in accord with its socio-democratic principles. Therefore, the authorities are committed to restore macroeconomic stability and place the economy on a path of sustained high growth to accelerate progress toward reaching the MDGs and middle-income status for Ghana. They request a PRGF arrangement to support their stabilization and reform program for 2009-12.

\section{Recent developments}

Ghana's economic performance improved markedly during 2001-06, with robust growth, a significant decline in inflation, and narrowing fiscal and current account deficits. Debt relief under the HIPC Initiative and MDRI improved the country's debt situation and freed resources for social spending. As a result, the incidence of poverty was significantly reduced along with improvement in other social indicators, including in education, health, and gender representation. Substantial progress was also made in structural reforms, particularly in the fiscal area, where revenue and expenditure management was strengthened; in the monetary area, which saw steady progress in liquidity management and eventual adoption of an inflation targeting (IT) framework; and in the financial sector, with important structural and institutional reforms to strengthen the system and increase its resilience and contribution to private sector development.

Since 2006, Ghana's economy came under stress from a succession of external and domestic shocks, including a severe drought, which triggered an acute energy crisis, and the global food and fuel price hikes. The energy crisis necessitated large scale investment in the thermopower generation, whereas food tariffs were reduced and energy subsidies increased in view of the social concerns about raising domestic prices in line with the sharply increasing international prices. The energy crisis in particular, made the implementation of the price adjustment mechanism very difficult. These, together with increased spending in the run up to the 2008 elections, resulted in higher fiscal and external current account deficits, a fall in reserves, a marked rise in inflation, and sharp exchange rate depreciation. However, real GDP growth in 2008 rose to a record high, supported by buoyant agricultural production and 
strong domestic demand.

Encouragingly, the banking system has remained sound, well capitalized, and fairly liquid, with a reduction in credit concentration, intensified competition, and expansion in branch networks. Thus far, the sector has not been significantly affected by the global crisis.

\section{The outlook for 2009 and the medium term}

Like other Sub-Saharan African countries, Ghana is being affected by the global economic downturn through the channels of remittances, portfolio investment flows, and FDI.

Nonetheless, these adverse developments are partly offset by favorable market conditions for cocoa and gold and the decline of oil import prices. Against this background, the growth deceleration in 2009 will be somewhat contained.

Over the medium term, the start of oil production in 2011 will have beneficial effects on growth, the fiscal situation, and the balance of payments. The authorities intend to manage prudently the oil resources and ensure their cautious phasing into the economy. They are fully aware of the challenges posed by increased oil revenue for macroeconomic management and will continue the national dialogue on the use of oil revenue while drawing on international best practices in this area. Non-oil GDP growth is expected to reach 6 percent on average during 2010-2014, while inflation is to be brought down gradually to 5 percent. The external current account deficit should narrow steadily to 6 percent by 2014 , and gross international reserves should recover to over 4 months of imports. The public debt will be stabilized and subsequently reduced.

\section{Policies for 2009}

The authorities are committed to restoration of macroeconomic stability, which they consider critical to achieving sustained growth and durable poverty reduction. Their strategy to this end is underpinned by fiscal discipline based on prudent expenditure management and enhanced revenue mobilization. In 2009, the fiscal deficit will be reduced by more than 5 percent of GDP to 9.4 percent through a combination of expenditure reductions and revenue-enhancing measures. Expenditure cuts will affect goods and services, transfers, and non-developmental capital spending. On the revenue side, intakes are being enhanced by increasing a range of taxes and fees and improving collection.

First quarter revenue and non-interest expenditures are broadly on track. To offset the higherthan projected interest costs, and achieve the targeted fiscal deficit, additional fiscal savings totaling at least 1.0 percent of GDP are being implemented. The projected financing gap beyond available domestic borrowing is expected to be covered by assistance from the World Bank and exceptional budget support from other development partners. Any shortfall in program grant and loan financing will be offset by spending cuts. 
A tight monetary policy will complement fiscal policy to ease pressure on the balance of payments and the exchange rate. The Bank of Ghana $(\mathrm{BoG})$ responded to the rising inflation by increasing its Prime Rate by a cumulative 350 basis points in 2008, and further by 150 basis points in February 2009. The BoG will use its IT regime to achieve disinflation, with the support of fiscal consolidation, and restore stability to the foreign exchange market. Inflation is projected to decline to $14 \frac{1}{2}$ percent by end-2009. With exceptional budget support and expected Fund financing, gross international reserves are projected at slightly below two months of import cover.

\section{The medium-term program}

Fiscal discipline will continue to underpin the authorities' medium-term objectives of achieving macroeconomic stability. The fiscal deficit will be progressively brought down to $4 \frac{1}{2}$ percent of GDP in 2011, and reduced further in subsequent years, driven by revenue mobilization and cuts in low-priority public spending, while shifting expenditure towards infrastructure investments. This will provide the needed support for monetary policy to reduce inflation to single digit from 2010 and stabilize price and exchange rate expectations. The BoG will continue to maintain a flexible exchange rate regime, consistent with the IT framework, with interventions limited to smoothing short-term fluctuations.

The authorities' structural reform agenda focuses on the fiscal area, public sector, debt management, energy sector, and financial sector. In the fiscal area, reforms aim at strengthening institutions, enhancing revenue mobilization, and reinforcing public expenditure management. Wide-ranging tax policy and administration reforms are planned, including streamlining exemptions, minimizing leakages, broadening the base of VAT and income tax, and modernizing and integrating revenue administration. Establishing a proper framework for the management of oil revenue will receive priority attention, including introducing appropriate revenue-management legislation and adherence to EITI principles. To strengthen fiscal discipline, fiscal responsibility laws are being developed to lay out rules to help achieve desired fiscal outcomes. Building on recent progress in PFM reforms, efforts will focus on addressing weaknesses in expenditure monitoring, managing commitments and arrears, establishing a cash management framework that will provide an early warning system, and improving selection and evaluation of investment projects.

The authorities intend to address the heavy burden of the public sector wage bill in the context of a comprehensive public sector reform program with World Bank assistance. Reform will focus, in particular, on the pay system and optimum enrollment levels. It will also address rigidities in the budget arising from statutory funds and committed expenditures by reviewing their legal provisions and management in order to enhance policy flexibility and efficiency. The authorities intend to address the financial difficulties of state-owned enterprises to minimize fiscal risks. They plan to move speedily to ensure completion of the study to review the financial status of all three power sector utilities and propose a restructuring and recovery program that will ensure their long-term viability. To address the 
cost of energy subsidies, the government intends to maintain the current system of costrecovery price adjustments for petroleum products and will adjust electricity tariffs to costrecovery levels, as needed, by end-2009. The restructuring of agencies that are recipient of government subsidies will be stepped up, with the view to liquidating nonviable ones and commercializing others, as necessary.

While the updated DSA shows improvement over last year's, the authorities recognize that there are vulnerabilities, particularly to oil revenues and fiscal performance, which they intend to address. A comprehensive debt management strategy will set a clear framework for borrowing and guide decisions regarding concessional financing as well as the financing of critical projects that may require state guarantee. The authorities will inform the Fund on projects being considered for nonconcessional financing, starting from the first program review, and request that limits on nonconcessional borrowing be set to accommodate those projects.

On the financial sector, the BoG has embarked on a comprehensive program of risk-based supervision and wide-ranging regulations which will underpin the development of robust and resilient financial sector and also address risks associated with weak governance structures. A recent increase in capital requirements was meant to strengthen banks as well as extend their reach in a rapidly growing economy. The domestic debt market is being actively developed to deepen financial markets and to improve the efficiency of the government's debt operations. Given the extensive changes in Ghana's financial sector since the last FSAP update, the authorities are interested in an early update.

\section{Conclusion}

My Ghanaian authorities are grateful to the Board and management for their support, which would catalyze assistance from other development partners and attract FDI. They reiterate their commitment to take the necessary measures to keep their program on track and enable them to achieve their socio-economic goals. They recognize that the global crisis reinforces the urgency to step up adjustment and reform policies and to mobilize external financing, consistent with a prudent debt strategy. While there are risks to the program, the authorities stand ready to adjust their policies, as necessary, to mitigate them in consultation with Fund staff. 